\title{
$q$-HERMITE POLYNOMIALS, BIORTHOGONAL RATIONAL FUNCTIONS, AND $q$-BETA INTEGRALS
}

\author{
M. E. H. ISMAIL AND D. R. MASSON
}

\begin{abstract}
We characterize the solutions of the indeterminate moment problem associated with the continuous $q$-Hermite polynomials when $q>1$ in terms of their Stieltjes transforms. The extremal measures are found explicitly. An analog of the Askey-Wilson integral is evaluated. It involves integrating a kernel, similar to the Askey-Wilson kernel, against any solution of the $q$-Hermite moment problem, provided that certain integrability conditions hold. This led to direct evaluation of several $q$-beta integrals and their discrete analogs as well as a generalization of Bailey's $6 \psi_{6}$ sum containing infinitely many parameters. A system of biorthogonal rational functions is also introduced.
\end{abstract}

\section{INTRODUCTION}

A basic hypergeometric function, with base $q$, is

$$
\begin{gathered}
{ }_{r} \phi_{s}\left(\begin{array}{l}
a_{1}, \ldots, a_{r} \\
b_{1}, \ldots, b_{s}
\end{array} \mid q, z\right)={ }_{r} \phi_{s}\left(a_{1}, \ldots, a_{r} ; b_{1}, \ldots, b_{s} ; q, z\right) \\
:=\sum_{n=0}^{\infty} \frac{\left(a_{1}, \ldots, a_{r}\right)_{n} z^{n}}{\left(q, b_{1}, \ldots, b_{s}\right)_{n}}\left[(-1)^{n} q^{n(n-1) / 2}\right]^{1+s-r},
\end{gathered}
$$

where the $q$-shifted factorial is

$$
\left(c_{1}, \ldots, c_{p}\right)_{n}=\left(c_{1}, \ldots, c_{p} ; q\right)_{n}:=\prod_{j=1}^{p} \prod_{k=1}^{n}\left(1-c_{j} q^{k-1}\right), \quad n=0,1, \ldots,
$$

and as usual empty sums are interpreted as 0 , empty products as unity. We will delete " $; q$ " from the shifted factorial notation (1.2) unless more than one basis is used in the same formula.

Received by the editors August 3,1993. Presented to the Society at the 99 th annual meeting in San Antonio, Texas, January 1993.

1991 Mathematics Subject Classification. Primary 33D45, 33D05; Secondary 42A65, 30E05.

Key words and phrases. Hamburger moment problem, indeterminate moment problem, Bailey's sum, Poisson kernel, biorthogonal rational functions, $q$-beta integrals, asymptotics, $q$-Hermite polynomials, theta functions, generating functions, Askey-Wilson integral.

Research of the first author was supported in part by NSF grant DMS 9203659.

Research of the second author was supported in part by NSERC grant A5384. 
R. Askey and J. Wilson [17] introduced the orthogonal polynomials

$$
p_{n}(x ; a, b, c, d)={ }_{4} \phi_{3}\left(\begin{array}{c|c}
q^{-n}, a b c d q^{n-1}, a z, a / z & q b, a c, a d
\end{array} \mid q\right),
$$

with

$$
z:=x-\sqrt{x^{2}-1}
$$

The Askey-Wilson polynomials are the most general orthogonal polynomials that share the properties of the more traditional orthogonal polynomials like the Legendre polynomials or the spherical harmonics [50]. They are eigenfunctions of a linear second order divided difference operator reminiscent of SturmLiouville operators. The Askey-Wilson polynomials also have a Rodrigues type representation. Their orthogonality relation is [17], [25]

$$
\begin{aligned}
\int_{0}^{\pi} p_{n}(\cos \theta ; & \left.a_{1}, a_{2}, a_{3}, a_{4}\right) p_{m}\left(\cos \theta ; a_{1}, a_{2}, a_{3}, a_{4}\right) \frac{\left(e^{2 i \theta}, e^{-2 i \theta}\right)_{\infty} d \theta}{\prod_{j=1}^{4}\left(a_{j} e^{i \theta}, a_{j} e^{-i \theta}\right)_{\infty}} \\
= & \frac{2 \pi a_{1}^{2 n}\left(a_{2} a_{3}, a_{2} a_{4}, a_{3} a_{4}, q\right)_{n}\left(1-a_{1} a_{2} a_{3} a_{4} q^{n-1}\right)}{\left(a_{1} a_{2}, a_{1} a_{3}, a_{1} a_{4}, a_{1} a_{2} a_{3} a_{4}\right)_{n}\left(1-a_{1} a_{2} a_{3} a_{4} q^{2 n-1}\right)} \\
& \times\left(a_{1} a_{2} a_{3} a_{4}\right)_{\infty} \delta_{m, n} /\left[(q)_{\infty} \prod_{1 \leq j<k \leq 4}\left(a_{j} a_{k}\right)_{\infty}\right],
\end{aligned}
$$

provided that $\left|a_{j}\right|<1, j=1,2,3,4$, and $-1<q<1$.

About a hundred years ago L.J. Rogers [8], [44], [45], [46] introduced a very interesting system of polynomials and used it to prove the Rogers-Ramanujan identities. The polynomial system is $\left\{C_{n}(x ; \beta \mid q)\right\}$

$$
C_{n}(\cos \theta, \beta \mid q)=\sum_{k=0}^{n} \frac{(\beta)_{k}(\beta)_{n-k}}{(q)_{k}(q)_{n-k}} e^{i(n-2 k) \theta} .
$$

The above polynomials have the generating function

$$
\sum_{n=0}^{\infty} C_{n}(\cos \theta, \beta \mid q) t^{n}=\left(t \beta e^{i \theta}, t \beta e^{-i \theta}\right)_{\infty} /\left(t e^{i \theta}, t e^{-i \theta}\right)_{\infty} .
$$

The $C_{n}$ 's are $q$-analogs of the ultraspherical polynomials (spherical harmonics) and contain an important special case, $\beta=0$, which is a $q$-analog of the Hermite polynomials. For $0<q<1$ set

$$
H_{n}(x \mid q):=(q)_{n} C_{n}(x ; 0 \mid q) .
$$

Thus

$$
\begin{gathered}
\sum_{n=0}^{\infty} H_{n}(\cos \theta \mid q) \frac{t^{n}}{(q)_{n}}=1 /\left(t e^{i \theta}, t e^{-i \theta}\right)_{\infty} \\
H_{n}(\cos \theta \mid q)=\sum_{k=0}^{n} \frac{(q)_{n}}{(q)_{k}(q)_{n-k}} e^{i(n-2 k) \theta} .
\end{gathered}
$$


The $C_{n}$ 's are called the continuous $q$-ultraspherical polynomials and the $H_{n}$ 's are called the continuous $q$-Hermite polynomials. The above notation and terminology first appeared in [14], [15] and has become standard since then. The measure that the $C_{n}$ 's are orthogonal with respect to was found in [14], [15] using asymptotic and moment methods and in [17] as the special case $a=-c=\sqrt{\beta}, b=-d=\sqrt{\beta q}$ of the Askey-Wilson polynomials (1.3).

Rogers solved the connection coefficient problem and the problem of linearization of products of continuous $q$-ultraspherical polynomials. He proved

$$
C_{m}(x ; \beta \mid q) C_{n}(x ; \beta \mid q)=\sum_{k=0}^{m \wedge n} a(k, m, n) C_{m+n-2 k}(x ; \beta \mid q),
$$

with $m \wedge n=\min (m, n)$ and

$$
a(k, m, n)=\frac{(\beta)_{m-k}(\beta)_{n-k}(q)_{m+n-2 k}(\beta)_{k}\left(\beta^{2}\right)_{m+n-k}\left(1-\beta q^{m+n-2 k}\right)}{\left(\beta^{2}\right)_{m+n-2 k}(q)_{m-k}(q)_{n-k}(q)_{k}(\beta q)_{m+n-k}(1-\beta)} .
$$

In particular,

$$
H_{m}(x \mid q) H_{n}(x \mid q)=\sum_{k=0}^{m \wedge n} \frac{(q)_{m}(q)_{n}}{(q)_{m-k}(q)_{n-k}(q)_{k}} H_{m+n-2 k}(x \mid q) .
$$

The key step in establishing (1.5) is the evaluation of the Askey-Wilson integral

$$
\begin{aligned}
(q)_{\infty} & \int_{0}^{\pi}\left[\left(e^{2 i \theta}, e^{-2 i \theta}\right)_{\infty} / \prod_{1 \leq j \leq 4}\left(a_{j} e^{i \theta}, a_{j} e^{-i \theta}\right)_{\infty}\right] d \theta \\
& =2 \pi\left(a_{1} a_{2} a_{3} a_{4}\right)_{\infty} / \prod_{1 \leq j<k \leq 4}\left(a_{j} a_{k}\right)_{\infty}, \quad-1<q<1 .
\end{aligned}
$$

The continuous $q$-Hermite polynomials are essentially the special case $a=$ $b=c=d=0$ of the Askey-Wilson polynomials (1.13). This may make it seem like the $H_{n}$ 's are the lowest level in a hierarchy of a four parameter family of polynomials. Ismail and Stanton [30] observed that the Askey-Wilson integral (1.14) actually follows from Rogers's linearization formula (1.13) and the orthogonality of the continuous $q$-Hermite polynomials. The orthogonality of the continuous $q$-Hermite polynomials follows from the Jacobi triple product identity [3], [7] or from a combination of an asymptotic analysis and a theorem of Markov [14]. Ismail, Stanton and Viennot [31] developed a combinatorial theory that leads to combinatorial interpretations of integrals like (1.14) with an arbitrary number of pairs of factors in the denominator of the integrand. Their results also give an evaluation of (1.14).

This paper started from an attempt to understand the structure of the continuous $q$-Hermite polynomials and the Askey-Wilson integral when $q>1$. The three term recurrence relation satisfied by $H_{n}(x \mid q)$ is

$$
2 x H_{n}(x \mid q)=H_{n+1}(x \mid q)+\left(1-q^{n}\right) H_{n-1}(x \mid q), \quad n>0 .
$$


When $q>1$ the $H_{n}$ 's are orthogonal on the imaginary axis, so we need to renormalize the polynomials in order to make them orthogonal on the real axis. The proper normalization is [12]

$$
h_{n}(x \mid q)=i^{-n} H_{n}(i x \mid 1 / q)
$$

which gives

$$
\begin{aligned}
h_{0}(x \mid q)=1, \quad h_{1}(x \mid q)=2 x, & \\
h_{n+1}(x \mid q)=2 x h_{n}(x \mid q)-q^{-n}\left(1-q^{n}\right) h_{n-1}(x \mid q), & \\
n>0, & 0<q<1 .
\end{aligned}
$$

We shall refer to the polynomials $h_{n}(x \mid q)$ as the $q^{-1}$-continuous Hermite polynomials, or the $q^{-1}$-Hermite polynomials.

The moment problem associated with $\left\{h_{n}(x \mid q)\right\}$ is indeterminate. To prove this it suffices to show that the corresponding orthonormal polynomial set $\left\{\tilde{h}_{n}(x)\right.$ $\left.=q^{n(n+1) / 4} h_{n}(x \mid q) / \sqrt{(q ; q)_{n}}\right\}$ is square summable (see [1, Theorem 1.3.1]). This easily follows from the asymptotics established in (2.9) of Section 2 which yields $\left|\tilde{h}_{n}(x)\right|^{2} \leq$ const. $|q|^{n / 2}$. Thus the $h_{n}$ 's are orthogonal with respect to infinitely many probability measures [1], [12], [47]. Among these measures there is a family of extremal measures $\{d \psi(x, \sigma)\}$ indexed by a parameter $\sigma$ for every real $\sigma$ including $\sigma= \pm \infty$. For every $\sigma \in[-\infty, \infty]$, the polynomials are complete in $L^{2}(d \psi(\sigma, x))$. In Section 6 we shall compute the Stieltjes transforms $\int_{-\infty}^{\infty} \frac{d \psi(t, \sigma)}{z-t}$ of all extremal measures with respect to which the $h_{n}$ 's are orthogonal. The identification of such Stieltjes transforms involves finding four entire functions $A(z), B(z), C(z), D(z)$, so that

$$
\frac{A(z)-\sigma(z) C(z)}{B(z)-\sigma(z) D(z)}=\int_{-\infty}^{\infty} \frac{d \psi(t ; \sigma)}{z-t} .
$$

For extremal measures $\sigma(z)$ is a real constant. Akhiezer [1] uses $N$-extremal to describe what Shohat and Tamarkin [47] refer to as extremal measures. We decided to follow the notation of Shohat and Tamarkin [47] throughout this work.

In Section 5 we compute $A(z), B(z), C(z)$, and $D(z)$. By computing each of the functions $A(z), B(z), C(z)$, and $D(z)$ in two different ways we are led to two known quartic transformations for theta functions, see (5.37) and (5.38), and to two new quartic transformations for ${ }_{2} \phi_{1}$ functions. The new quartic transformations are stated in (5.46) and (5.47). In Section 6 we invert the Stieltjes transform (1.17) and find the one parameter family of extremal measures. The inversion uses properties of theta functions because $B(z)$ and $D(z)$ are theta functions. The key idea is a representation of $\sigma$ as a quotient of two theta functions of a parameter $\eta$, say. This enables us to find all zeros of $B(z)-\sigma D(z)$ using addition theorems and product formulas for theta functions. This is reminiscent of the case of trigonometric functions when $\sigma=\cot z$ identifies the zeros of $\cos \phi-\sigma \sin \phi$ with the zeros of $\sin (z-\phi)$. In general $\sigma(z)$ in (1.17) is a function analytic in the open upper half plane and maps this region into the closed lower half plane. 
We shall also evaluate the following analog of the Askey-Wilson integral

$$
I\left(t_{1}, t_{2}, t_{3}, t_{4}\right)=\int_{-\infty}^{\infty} \prod_{j=1}^{4}\left(-t_{j}\left(\sqrt{x^{2}+1}+x\right), t_{j}\left(\sqrt{x^{2}+1}-x\right)\right)_{\infty} d \psi(x) .
$$

This will be done in Section 3. It is interesting to note that our derivation uses only the integrability of the integrand in (1.18) with respect to $d \psi$ and the fact that the $h_{n}$ 's are orthogonal with respect to $d \psi$.

The measures $\{d \psi\}$ are normalized to have total mass $=1$. The extremal measures are purely discrete. For extremal measures the normalization $\int_{-\infty}^{\infty} d \psi(x ; \sigma)=1$ implies the Jacobi triple product identity, while the evaluation (3.8) of $I\left(t_{1}, t_{2}, t_{3}, t_{4}\right)$ of $(1.18)$ is Bailey's ${ }_{6} \psi_{6}$ sum. This will also be discussed in Section 3. Askey [12] proved that the $h_{n}$ 's are orthogonal on $(-\infty, \infty)$ with respect to the measure

$$
\left\{\sqrt{1+x^{2}} \prod_{n=0}^{\infty}\left[1+2\left(2 x^{2}+1\right) q^{n+1}+q^{2 n+2}\right]\right\}^{-1} d x .
$$

Later Askey [10] evaluated the integral $I$ in $(1.18)$ when $d \psi(x) / d x$ is his weight function. Our analysis gives Askey's second integral from his earlier weight function. This will be done in Section 7. We shall also consider the measures $d \psi$ corresponding to the choice

$$
\sigma(z)=c, \Im z>0, \quad \text { with } \overline{\sigma(z)}=\sigma(\bar{z}),
$$

where $c$ is a nonreal constant. The above measures are, in some sense, close to being extremal. When such measures were inserted in (1.18) we were led to new $q$-beta integrals.

In Section 3 the integrals (1.18) are used to introduce rational functions $\left\{\varphi_{n}\right\}$ biorthogonal with respect to $w\left(x ; t_{1}, t_{2}, t_{3}, t_{4}\right) d \psi(x)$,

$$
w(x)=w\left(x ; t_{1}, t_{2}, t_{3}, t_{4}\right):=\prod_{j=1}^{4}\left(-t_{j}\left(x+\sqrt{x^{2}+1}\right), t_{j}\left(\sqrt{x^{2}+1}-x\right)\right)_{\infty} .
$$

The biorthogonal rational functions are

$$
\begin{aligned}
& \varphi_{n}\left(\sinh \xi ; t_{1}, t_{2}, t_{3}, t_{4}\right) \\
& \quad={ }_{4} \phi_{3}\left(\begin{array}{c|c}
q^{-n},-t_{1} t_{2} q^{n-2},-t_{1} t_{3} / q,-t_{1} t_{4} / q & q, q) . \\
-t_{1} e^{\xi}, t_{1} e^{-\xi}, t_{1} t_{2} t_{3} t_{4} q^{-3} & q
\end{array}\right.
\end{aligned}
$$

They satisfy the orthogonality relation

$$
\int_{-\infty}^{\infty} \varphi_{m}\left(x ; t_{1}, t_{2}, t_{3}, t_{4}\right) \varphi_{n}\left(x ; t_{2}, t_{1}, t_{3}, t_{4}\right) w(x) d \psi(x)=g_{n} \delta_{m, n},
$$


where $\psi$ is the probability measure with respect to which the $h_{n}$ 's are orthogonal, $w(x)$ is as in (1.19), and $g_{n}$ is

$$
\begin{aligned}
g_{n}= & \frac{1+t_{1} t_{2} q^{n-2}}{1+t_{1} t_{2} q^{2 n-2}} \frac{\left(t_{1} t_{2} t_{3} t_{4} q^{-3}\right)^{n}\left(q,-q^{2} / t_{3} t_{4}\right)_{n}\left(-t_{1} t_{2} q^{n-1}\right)_{\infty}}{\left(t_{1} t_{2} t_{3} t_{4} q^{-3}\right)_{n}} \\
& \times \frac{\left(-t_{1} t_{3} / q,-t_{1} t_{4} / q,-t_{2} t_{3} / q,-t_{2} t_{4} / q,-t_{3} t_{4} / q\right)_{\infty}}{\left(t_{1} t_{2} t_{3} t_{4} q^{-3}\right)_{\infty}} .
\end{aligned}
$$

After we showed this work to Mizan Rahman, he kindly pointed out that he [43] discovered the orthogonality relation (1.21) in the special case when $d \psi$ is the above-mentioned absolutely continuous measure given by Askey in [12]. For a general setting and applications of biorthogonality we refer the interested reader to the recent book [19].

The spectral theory of orthogonal polynomials is essentially the spectral theory of symmetric second order difference operators or infinite symmetric Jacobi matrices [51]. The Jacobi matrix associated with (1.16) is

$$
\begin{array}{r}
J=\left(a_{m n}\right), \quad a_{m, m+1}=a_{m+1, m}=\frac{1}{2} \sqrt{q^{-m-1}-1}, \\
\quad \text { otherwise } a_{m, n}=0, \quad m, n \geq 0 .
\end{array}
$$

The operator $J$ is unbounded and closed on $l^{2}$ but does not have a unique selfadjoint extension. The extremal measures are precisely the spectral measures corresponding to orthogonal spectral resolutions of the identity [1]. The operator $J$ is a Schrödinger operator and our analysis seems to the first complete analysis of an unbounded Schrödinger operator not possessing a unique self-adjoint extension.

In Section 2 we find the Poisson kernel of the $h_{n}$ 's. We can obtain additional generating functions using a technique similar to what Ismail and Stanton used in [30] and was also used by Al-Salam and Ismail in the later work [4].

In Section 4 we indicate how the pointwise asymptotics of rational functions may give a clue about a possible measure with respect to which they are biorthogonal. This point of view is illustrated by two examples. The first is a system of biorthogonal rational functions introduced in [6] and the second is the system $\left\{\varphi_{n}\left(x ; t_{1}, t_{2}, t_{3}, t_{4}\right)\right\}$ of (1.20). In the case of the $\varphi_{n}$ 's this leads to a discrete biorthogonality relation, see (4.22), different from the biorthogonality relation (1.21). Although several explicit biorthogonal systems of biorthogonal functions are known there is no general theory available for such systems. For recent biorthogonal systems we refer the interested reader to [5], [41], and [42].

Section 7 contains various applications of the main results of the paper. For example we combine general results from the theory of the Hamburger moment problem with our evaluation of the integral in (1.18) to obtain a generalization of Bailey's ${ }_{6} \psi_{6}$ sum. This generalization is stated as Theorem 7.5. In addition we find explicitly a spectral measure $d \psi$ for the continuous $q^{-1}$-Hermite polynomials with an absolutely continuous component supported on $(-\infty, 0]$ and an infinite discrete part with masses located in $[0, \infty)$. With this measure the evaluation of $I\left(t_{1}, t_{2}, t_{3}, t_{4}\right)$ gives an identity involving a sum and an integral. We also discuss other identities. 
Section 8 is devoted to a generalization of the ${ }_{6} \psi_{6}$ to finitely and infinitely many parameters. These results are our Theorems 8.1 and 8.2. Chapter 9 contains an alternate derivation of the extremal measures of the $q^{-1}$-Hermite polynomials which uses only the quasiperiodicity of the theta functions. In Section 9 we introduce the Askey-Wilson operator whose action lowers the degree of a $q^{-1}$-Hermite polynomial by 1 and we also evaluate a new $q$-beta integral. We also discover two quadratic transformations for theta functions, see (9.28) and (9.29).

This work is the first where an indeterminate moment problem is solved completely and all extremal measures are computed explicitly. Other recent works dealing with specific indeterminate moment problems are [37], [18] and [22]. We were fortunate to study the $q^{-1}$-Hermite moment problem because the $B$ and $D$ functions in (1.17) were theta functions and we were able to take advantage of the rich theory of theta functions including addition and product formulas. Indeed theta functions saved the day.

Some of the results of this work were announced in [29].

\section{GeNERATING FUNCTIONS}

The main results of this section are formulas $(2.4),(2.8)$, which are the building blocks of the rest of this work.

It is easy to see that

$$
(1 / q ; 1 / q)_{j}=(q ; q)_{j}(-1)^{j} q^{-j(j+1) / 2} .
$$

Substitute from (2.1) into (1.10) and then apply (1.15) to obtain the explicit representation

$$
h_{n}(x \mid q)=\sum_{k=0}^{n} \frac{(q)_{n}}{(q)_{k}(q)_{n-k}}(-1)^{k} q^{k(k-n)}\left(x+\sqrt{x^{2}+1}\right)^{n-2 k} .
$$

To derive a generating function for $\left\{h_{n}(x \mid q)\right\}$ multiply (2.2) by $t^{n} q^{n^{2} / 2} /(q)_{n}$ and add for $n \geq 0$. The result after replacing $n$ by $n+k$ is

$$
\sum_{n=0}^{\infty} \frac{t^{n} q^{n^{2} / 2}}{(q)_{n}} h_{n}(x \mid q)=\sum_{n, k=0}^{\infty} \frac{t^{n+k} q^{\left(n^{2}+k^{2}\right) / 2}(-1)^{k}}{(q)_{k}(q)_{n}}\left(x+\sqrt{x^{2}+1}\right)^{n-k} .
$$

The right-hand side can now be summed by Euler's formula

$$
\sum_{n=0}^{\infty} q^{n(n-1) / 2} \frac{(-z)^{n}}{(q)_{n}}=(z)_{\infty} .
$$

Thus we have established the generating function

$$
\sum_{n=0}^{\infty} \frac{t^{n} q^{n(n-1) / 2}}{(q)_{n}} h_{n}(x \mid q)=\left(-t\left(x+\sqrt{x^{2}+1}\right), t\left(\sqrt{x^{2}+1}-x\right)\right)_{\infty},
$$

since $1 /\left(\sqrt{x^{2}+1}+x\right)=\sqrt{x^{2}+1}-x$.

We now derive a Poisson kernel for $\left\{h_{n}(x \mid q)\right\}$ from Rogers's linearization formula a lá Ismail and Stanton [30]. Another derivation is in [2]. Replace $x$ 
by $i x$ and $q$ by $1 / q$ in (1.13) and use (2.1) and (1.15). The result is

$$
\begin{aligned}
q^{\left(\begin{array}{c}
m \\
2
\end{array}\right)} & \frac{h_{m}(x \mid q)}{(q)_{m}} q^{\left(\begin{array}{c}
n \\
2
\end{array}\right)} \frac{h_{n}(x \mid q)}{(q)_{n}} \\
= & \sum_{k=0}^{m \wedge n} \frac{q^{-n-m+\left(\begin{array}{c}
c+1 \\
2
\end{array}\right)+\left(\begin{array}{c}
m-k+1 \\
2
\end{array}\right)+\left(\begin{array}{c}
n-k+1 \\
2
\end{array}\right)}}{(q)_{k}(q)_{m-k}(q)_{n-k}} h_{m+n-2 k}(x \mid q) .
\end{aligned}
$$

Multiply (2.5) by $s^{m} t^{n}$ and sum over $m$ and $n$ for $m \geq 0, n \geq 0$. Using (2.4) we see that the left side sums to

$$
\left(-s\left(x+\sqrt{x^{2}+1}\right), s\left(\sqrt{x^{2}+1}-x\right),-t\left(x+\sqrt{x^{2}+1}\right), t\left(\sqrt{x^{2}+1}-x\right)\right)_{\infty} .
$$

On the other hand, the right side, after interchanging the $m$ and $n$ sums with the $k$ sum, then replacing $m$ and $n$ by $m+k$ and $n+k$, respectively, becomes

$$
\sum_{m, n=0}^{\infty} \frac{s^{m} t^{n}}{(q)_{m}(q)_{n}} q^{\left(\begin{array}{c}
m \\
2
\end{array}\right)+\left(\begin{array}{c}
n \\
2
\end{array}\right) h_{m+n}}(x \mid q) \sum_{k=0}^{\infty} \frac{(s t)^{k} q^{\left(\begin{array}{c}
k \\
2
\end{array}\right)-k}}{(q)_{k}} \text {. }
$$

Now (2.3) and rearrangement of series reduce the above expression to

$$
(-s t / q)_{\infty} \sum_{j=0}^{\infty} h_{j}(x \mid q) \sum_{n=0}^{j} \frac{s^{j-n} t^{n}}{(q)_{n}(q)_{j-n}} q^{\left(\begin{array}{c}
n \\
2
\end{array}\right)+\left(\begin{array}{c}
j-n \\
2
\end{array}\right) .}
$$

At this stage we found it more convenient to set

$$
s=R\left(y+\sqrt{y^{2}+1}\right), \quad t=-R\left(\sqrt{y^{2}+1}-y\right),
$$

and

$$
x=\sinh \xi, \quad y=\sinh \eta .
$$

The above calculations lead to

$$
\begin{aligned}
& \left(-R e^{\xi+\eta}, R e^{\eta-\xi}, R e^{\xi-\eta},-R e^{-\xi-\eta}\right)_{\infty} /\left(R^{2} / q\right)_{\infty} \\
& \quad=\sum_{j=0}^{\infty} h_{j}(\sinh \eta \mid q) R^{j} q^{j(j-1) / 2} \sum_{n=0}^{j} \frac{q^{n(n-j)}(-1)^{n} e^{(j-2 n) \xi}}{(q)_{n}(q)_{j-n}} .
\end{aligned}
$$

This establishes the following theorem.

Theorem 2.1. The Poisson kernel (or the q-Mehler formula) for the continuous $q^{-1}$-Hermite polynomials is

$$
\begin{aligned}
& \sum_{n=0}^{\infty} h_{n}(\sinh \xi \mid q) h_{n}(\sinh \eta \mid q) \frac{q^{n(n-1) / 2}}{(q)_{n}} R^{n} \\
& \quad=\left(-R e^{\xi+\eta},-R e^{-\xi-\eta}, R e^{\xi-\eta}, R e^{\eta-\xi}\right)_{\infty} /\left(R^{2} / q\right)_{\infty} .
\end{aligned}
$$

The Poisson kernel is the left-hand side of (2.8) with $R$ replaced by $q R$, but we rescaled it to make the result nicer. We can obtain additional generating functions using a technique similar to what Ismail and Stanton used in [30] and was also used by Al-Salam and Ismail in the later work [4]. These generating functions are $q$-analogs of what typically follows from the Kibble-Slepian formula, [23], [33], [36], [48]. Louck [36] pointed out that the Kibble-Slepian 
formula follows from the invariance of the Laplacian under the orthogonal group while Slepian [48] used the Fourier transform to prove the same result. Neither technique has been developed yet for $q$-series. In the absence of a $q$-Kibble-Slepian formula the multilinear generating functions may be of some interest in themselves and may point out where to look for a $q$-analog of the Kibble-Slepian formula. The details will appear elsewhere.

The bilinear generating function $(2.8)$ can be used to determine the large $n$ asymptotics of $h_{n}(x \mid q)$. To see this let $\xi=\eta$ and apply Darboux's asymptotic method. The result is

$$
\begin{aligned}
h_{n}^{2}(\sinh \xi \mid q) q^{n(n-1) / 2} \approx \frac{q^{-n / 2}}{2} & {\left[\left(-\sqrt{q} e^{2 \xi},-\sqrt{q} e^{-2 \xi}, \sqrt{q}, \sqrt{q} ; q\right)_{\infty}\right.} \\
& \left.+(-1)^{n}\left(\sqrt{q} e^{2 \xi}, \sqrt{q} e^{-2 \xi},-\sqrt{q},-\sqrt{q} ; q\right)_{\infty}\right] .
\end{aligned}
$$

Thus

$$
\begin{aligned}
h_{2 n}^{2}(\sinh \xi \mid q) \approx \frac{q^{-2 n^{2}}}{2}[ & \left(-\sqrt{q} e^{2 \xi},-\sqrt{q} e^{-2 \xi}, \sqrt{q}, \sqrt{q} ; q\right)_{\infty} \\
& \left.+\left(\sqrt{q} e^{2 \xi}, \sqrt{q} e^{-2 \xi},-\sqrt{q},-\sqrt{q} ; q\right)_{\infty}\right],
\end{aligned}
$$

and

(2.11)

$$
\begin{aligned}
h_{2 n+1}^{2}(\sinh \xi \mid q) \approx \frac{q^{-(2 n+1)^{2} / 2}}{2}[ & \left(-\sqrt{q} e^{2 \xi},-\sqrt{q} e^{-2 \xi}, \sqrt{q}, \sqrt{q} ; q\right)_{\infty} \\
& \left.-\left(\sqrt{q} e^{2 \xi}, \sqrt{q} e^{-2 \xi},-\sqrt{q},-\sqrt{q} ; q\right)_{\infty}\right] .
\end{aligned}
$$

We shall comment on (2.10) and (2.11) in Section 9.

Another application of (2.8) is to compute the discrete masses of the extremal measures from the knowledge of their spectrum. The details follow (6.29).

\section{SOME BIORTHOGONAL RATIONAL FUNCTIONS}

As we pointed out in the introduction the orthogonality of the Askey-Wilson polynomials (1.3) easily follows from the evaluation of the Askey-Wilson integral (1.14). To prove the biorthogonality of the rational functions (1.19), we first evaluate the integral (1.18), namely

$$
\begin{aligned}
I & =I\left(t_{1}, t_{2}, t_{3}, t_{4}\right) \\
& :=\int_{-\infty}^{\infty} \prod_{j=1}^{4}\left(-t_{j}\left(x+\sqrt{x^{2}+1}\right), t_{j}\left(\sqrt{x^{2}+1}-x\right)\right)_{\infty} d \psi(x),
\end{aligned}
$$

where the $h_{n}$ 's are orthogonal with respect to $d \psi$, provided that the integral in (3.1) exists. One such $\psi$ is a jump function $\mu$ of Section 6 with jumps at $\pm x_{n}$

$$
x_{n}=\frac{1}{2}\left(q^{-n-1 / 2}-q^{n+1 / 2}\right), \quad n=0,1, \ldots
$$


with masses

$$
\mu\left( \pm x_{n}+0^{+}\right)-\mu\left( \pm x_{n}-0^{+}\right)=\frac{\left(q ; q^{2}\right)_{\infty}}{2\left(q^{2} ; q^{2}\right)_{\infty}}\left(1+q^{2 n+1}\right) q^{2 n^{2}+n}
$$

$$
n=0,1, \ldots
$$

The value of $I$ is given in (3.8). The orthogonality relation for $\left\{h_{n}(x \mid q)\right\}$ is

$$
\int_{-\infty}^{\infty} h_{m}(x \mid q) h_{n}(x \mid q) d \psi(x)=q^{-n(n+1) / 2}(q)_{n} \delta_{m, n} .
$$

In Section 6, we shall prove that $d \mu$ is an extremal measure for $\left\{h_{n}(x \mid q)\right\}$.

Theorem 3.1. The integral $I\left(t_{1}, t_{2}, t_{3}, t_{4}\right)$ of (3.1) is given by (3.8).

Proof. From (2.5) we obtain

$$
\begin{aligned}
& q^{\left(\begin{array}{c}
s \\
2
\end{array}\right)+\left(\begin{array}{c}
m \\
2
\end{array}\right)+\left(\begin{array}{c}
n \\
2
\end{array}\right)} \frac{h_{m}(x \mid q) h_{n}(x \mid q) h_{s}(x \mid q)}{(q)_{m}(q)_{n}(q)_{s}} \\
&=\sum_{k=0}^{m \wedge n} \frac{q^{-k+\left(\begin{array}{c}
k \\
2
\end{array}\right)+\left(\begin{array}{c}
m-k \\
2
\end{array}\right)+\left(\begin{array}{c}
n-k \\
2
\end{array}\right)-\left(\begin{array}{c}
m+n-2 k \\
2
\end{array}\right)}}{(q)_{k}(q)_{m-k}(q)_{n-k}}(q)_{m+n-2 k} \\
& \times \sum_{j=0}^{s \wedge(m-n-2 k)} \frac{q^{-j+\left(\begin{array}{c}
j \\
2
\end{array}\right)+\left(\begin{array}{c}
s-j \\
2
\end{array}\right)+\left(\begin{array}{c}
m+n-2 k-j \\
2
\end{array}\right)}}{(q)_{j}(q)_{s-j}(q)_{m+n-2 k-j}} h_{m+n+s-2 k-2 j}(x \mid q) .
\end{aligned}
$$

Multiply both sides by $t_{1}^{r} t_{2}^{s} t_{3}^{m} t_{4}^{n} q^{\left(\begin{array}{l}r \\ 2\end{array}\right)} h_{r}(x) /(q)_{r}$, integrate with respect to $d \psi(x)$, then add the results for all $m, n, r, s \geq 0$. The orthogonality relation (3.4) forces $m+n+s-2 k-2 j$ to equal $r$ and all the other terms contribute zeros to the sum. Thus $j=-k+(m+n+s-r) / 2$. The sums of integrals of the left sides is $I\left(t_{1}, t_{2}, t_{3}, t_{4}\right)$ as can be seen from (2.4) and the Lebesgue convergence theorem. Therefore we have

$$
\begin{aligned}
I= & \sum_{k, m, n, r, s=0}^{\infty} \frac{q^{\left(\begin{array}{c}
k \\
2
\end{array}\right)+\left(\begin{array}{c}
m-k \\
2
\end{array}\right)+\left(\begin{array}{c}
n-k \\
2
\end{array}\right)-\left(\begin{array}{c}
m+n-2 k \\
2
\end{array}\right)+\left(\begin{array}{c}
-k+(m+n+s-r) / 2 \\
2
\end{array}\right)}}{(q)_{k}(q)_{m-k}(q)_{n-k}(q)_{-k+(m+n+s-r) / 2}} \\
& \times \frac{q^{\left(\begin{array}{c}
k+(s+r-m-n) / 2 \\
2
\end{array}\right)+\left(\begin{array}{c}
-k+(m+n+r-s) / 2 \\
2
\end{array}\right)+k-(r+m+n+s) / 2}}{(q)_{k+(s+r-m-n) / 2}(q)-k+(m+n+r-s) / 2} \\
& \times(q)_{m+n-2 k} q^{-(m+n+r+s) / 2} t_{1}^{r} t_{2}^{s} t_{3}^{m} t_{4}^{n} .
\end{aligned}
$$

Replace $m$ and $n$ by $m+k$ and $n+k$, respectively, to get

$$
\begin{aligned}
& I=\sum_{k, m, n, r, s=0}^{\infty} \frac{q^{\left(\begin{array}{l}
k \\
2
\end{array}\right)+\left(\begin{array}{c}
(m+n+s-r) / 2 \\
2
\end{array}\right)+\left(\begin{array}{c}
(r+s-m-n) / 2 \\
2
\end{array}\right)+\left(\begin{array}{c}
(m+n+r-s) / 2 \\
2
\end{array}\right)}}{(q)_{k}(q)_{m}(q)_{n}(q)_{(m+n+s-r) / 2}(q)_{(r+s-m-n) / 2}} \\
& \times \frac{(q)_{m+n} q^{-m n-k-(m+n+r+s) / 2}}{(q)_{(m+n+r-s) / 2}} t_{1}^{r} t_{2}^{s} t_{3}^{m+k} t_{4}^{n+k} \text {. }
\end{aligned}
$$


The above multisums can be greatly simplified by the introduction of the new summation indices

$$
\alpha:=(m+n+s-r) / 2, \quad \beta:=(m+n+r-s) / 2, \quad \gamma:=(r+s-m-n) / 2
$$

instead of $n, r, s$, so our new summation indices are $k, m, \alpha, \beta, \gamma$. Clearly

$$
r=\beta+\gamma, \quad s=\alpha+\gamma, n=\alpha+\beta-m .
$$

Thus

$$
\begin{aligned}
I= & \sum_{k, m, \alpha, \beta, \gamma=0}^{\infty} \frac{q^{\left(\begin{array}{c}
k \\
2
\end{array}\right)+\left(\begin{array}{l}
\alpha \\
2
\end{array}\right)+\left(\begin{array}{l}
\gamma \\
2
\end{array}\right)+\left(\begin{array}{c}
\beta \\
2
\end{array}\right)+m(m-\alpha-\beta)-k-\alpha-\beta-\gamma}}{(q)_{k}(q)_{m}(q)_{\alpha+\beta-m}(q)_{\alpha}(q)_{\gamma}(q)_{\beta}} \\
& \times(q)_{\alpha+\beta} t_{1}^{\beta+\gamma} t_{2}^{\alpha+\gamma} t_{3}^{m+k} t_{4}^{\alpha+\beta+k-m} .
\end{aligned}
$$

The $\gamma$ sum is $\left(-t_{1} t_{2} / q\right)_{\infty}$ while the $k$ sum is $\left(-t_{3} t_{4} / q\right)_{\infty}$. This follows from (2.3). This reduces $I$ to a triple sum. Now replace $\alpha+\beta$ by $p$ to express $I$ as

$$
\begin{aligned}
I /\left(-t_{1} t_{2} / q,-t_{3} t_{4} / q\right)_{\infty}= & \sum_{p=0}^{\infty} \frac{\left(t_{1} t_{4} / q\right)^{p}}{(q)_{p}} q^{\left(\begin{array}{l}
p \\
2
\end{array}\right)} \sum_{m=0}^{p} \frac{(q)_{p}\left(t_{3} / t_{4}\right)^{m}}{(q)_{m}(q)_{p-m}} q^{m(m-p)} \\
& \times \sum_{\alpha=0}^{p} \frac{(q)_{p}\left(t_{2} / t_{1}\right)^{\alpha}}{(q)_{\alpha}(q)_{p-\alpha}} q^{\alpha(\alpha-p)}
\end{aligned}
$$

To evaluate the above triple sum we need to identify it with a Poisson kernel for $\left\{h_{n}(x \mid q)\right\}$. This identification can be achieved by setting

$$
t_{1}=\sqrt{q} T e^{\xi}, \quad t_{2}=-\sqrt{q} T e^{-\xi}, \quad t_{3}=-R \sqrt{q} e^{-\eta}, \quad t_{4}=R \sqrt{q} e^{\eta} .
$$

This leads to

$$
\begin{aligned}
I /\left(-t_{1} t_{2} / q,-t_{3} t_{4} / q\right)_{\infty} & =\sum_{p=0}^{\infty} \frac{(R T)^{p} q^{\left(\begin{array}{l}
p \\
2
\end{array}\right)}}{(q)_{p}} h_{p}(\sinh \xi \mid q) h_{p}(\sinh \eta \mid q) \\
& =\frac{\left(-R T e^{\xi+\eta},-R T e^{-\xi-\eta}, R T e^{\xi-\eta}, R T e^{\eta-\xi}\right)_{\infty}}{\left(R^{2} T^{2} / q\right)_{\infty}} \\
& =\frac{\left(-t_{1} t_{4} / q,-t_{2} t_{3} / q,-t_{1} t_{3} / q,-t_{2} t_{4} / q\right)_{\infty}}{\left(t_{1} t_{2} t_{3} t_{4} / q^{3}\right)_{\infty}}
\end{aligned}
$$

Therefore

$$
I\left(t_{1}, t_{2}, t_{3}, t_{4}\right)=\left[\prod_{1 \leq j<k \leq 4}\left(-t_{j} t_{k} / q\right)_{\infty}\right] /\left(t_{1} t_{2} t_{3} t_{4} q^{-3}\right)_{\infty} .
$$

This completes the evaluation of (3.1) and the proof of Theorem 3.1 is complete.

It is clear that (3.8) gives a summation theorem for a combination of basic hypergeometric functions. To identify the functions involved we expand the 
right side of (3.1) using (3.2) and (3.3). The result is

$$
\begin{aligned}
2\left(q^{2} ; q^{2}\right)_{\infty} & I\left(t_{1}, t_{2}, t_{3}, t_{4}\right) /\left(q ; q^{2}\right)_{\infty} \\
= & \sum_{n=0}^{\infty} \prod_{j=1}^{4}\left(-t_{j} q^{-n-1 / 2}, t_{j} q^{n+1 / 2}\right)_{\infty} q^{2 n^{2}+n}\left(1+q^{2 n+1}\right) \\
& + \text { a similar term with }\left(t_{1}, t_{2}, t_{3}, t_{4}\right) \text { replaced by }\left(-t_{1},-t_{2},-t_{3},-t_{4}\right) . \\
= & \prod_{j=1}^{4}\left(-t_{j} q^{-1 / 2}, t_{j} q^{1 / 2}\right)_{\infty} \sum_{n=0}^{\infty}\left[\prod_{j=1}^{4} \frac{\left(-q^{3 / 2} / t_{j}\right)_{n}}{\left(t_{j} q^{1 / 2}\right)_{n}}\right]\left(t_{1} t_{2} t_{3} t_{4} / q^{3}\right)^{n}\left(1+q^{2 n+1}\right) \\
& + \text { a similar term with }\left(t_{1}, t_{2}, t_{3}, t_{4}\right) \text { replaced by }\left(-t_{1},-t_{2},-t_{3},-t_{4}\right) .
\end{aligned}
$$

Therefore

$$
\begin{aligned}
& \frac{2\left(q^{4} ; q^{2}\right)_{\infty}}{\left(q^{3} ; q^{2}\right)_{\infty}} I\left(t_{1}, t_{2}, t_{3}, t_{4}\right)=\prod_{j=1}^{4}\left(-t_{j} q^{-1 / 2}, t_{j} q^{1 / 2}\right)_{\infty} \\
& \times{ }_{8} \phi_{7}\left(\begin{array}{c}
-q, i q^{3 / 2},-i q^{3 / 2},-q^{3 / 2} / t_{1},-q^{3 / 2} / t_{2},-q^{3 / 2} / t_{3},-q^{3 / 2} / t_{4}, q \\
i q^{1 / 2},-i q^{1 / 2}, t_{1} q^{1 / 2}, t_{2} q^{1 / 2}, t_{3} q^{1 / 2}, t_{4} q^{1 / 2},-q
\end{array} \mid\right. \\
& \left.\quad q, t_{1} t_{2} t_{3} t_{4} q^{-3}\right)
\end{aligned}
$$

+ interchange $t_{j}$ with $-t_{j}, j=1,2,3,4$.

This gives the summation formula

$$
\begin{aligned}
& \prod_{j=1}^{4}\left(-t_{j} q^{-1 / 2}, t_{j} q^{1 / 2}\right)_{\infty} \\
& \times{ }_{8} \phi_{7}\left(\begin{array}{c}
-q, i q^{3 / 2},-i q^{3 / 2},-q^{3 / 2} / t_{1},-q^{3 / 2} / t_{2},-q^{3 / 2} / t_{3},-q^{3 / 2} / t_{4}, q \\
i q^{1 / 2},-i q^{1 / 2}, t_{1} q^{1 / 2}, t_{2} q^{1 / 2}, t_{3} q^{1 / 2}, t_{4} q^{1 / 2},-q
\end{array} \mid\right. \\
& \left.q, t_{1} t_{2} t_{3} t_{4} q^{-3}\right)
\end{aligned}
$$

+ a similar term with $t_{j}$ replaced by $-t_{j}, 1 \leq j \leq 4$.

$$
\frac{2\left(q^{4} ; q^{2}\right)_{\infty}}{\left(q^{3} ; q^{2}\right)_{\infty}}\left[\prod_{1 \leq j<k \leq 4}\left(-t_{j} t_{k} / q\right)_{\infty}\right]\left(t_{1} t_{2} t_{3} t_{4} q^{-3}\right)_{\infty}^{-1} .
$$

We shall discuss other summation theorems that arise from (3.8) in Sections 7 and 8. See also (3.18).

We now prove the biorthogonality of the rational functions (1.20). Recall that

$$
\varphi_{n}\left(\sinh \xi ; t_{1}, t_{2}, t_{3}, t_{4}\right)={ }_{4} \phi_{3}\left(\begin{array}{c|c}
q^{-n},-t_{1} t_{2} q^{n-2},-t_{1} t_{3} / q,-t_{1} t_{4} / q \\
-t_{1} e^{\xi}, t_{1} e^{-\xi}, t_{1} t_{2} t_{3} t_{4} q^{-3}
\end{array} \mid q, q\right) .
$$


Theorem 3.2. The functions $\left\{\varphi_{n}\right\}$ satisfy the biorthogonality relation (1.21) with $g_{n}$ as in (1.22).

Proof. Consider the integrals

$$
\begin{aligned}
J_{m, n}:= & \int_{-\infty}^{\infty} \frac{\varphi_{n}\left(x ; t_{1}, t_{2}, t_{3}, t_{4}\right)}{\left(-t_{2}\left(x+\sqrt{x^{2}+1}\right), t_{2}\left(\sqrt{x^{2}+1}-x\right)\right)_{m}} \\
& \times \prod_{j=1}^{4}\left(-t_{j}\left(x+\sqrt{x^{2}+1}\right), t_{j}\left(\sqrt{x^{2}+1}-\right)\right)_{\infty} d \psi(x) .
\end{aligned}
$$

We have

$$
\begin{aligned}
J_{m, n}= & \sum_{k=0}^{n} \frac{\left(q^{-n},-t_{1} t_{2} q^{n-2},-t_{1} t_{3} / q,-t_{1} t_{4} / q\right)_{k}}{\left(q, t_{1} t_{2} t_{3} t_{4} q^{-3}\right)_{k}} q^{k} I\left(t_{1} q^{k}, t_{2} q^{m}, t_{3}, t_{4}\right) \\
= & \frac{\left(-t_{3} t_{4} / q,-t_{2} t_{3} q^{m-1},-t_{2} t_{4} q^{m-1},-t_{1} t_{2} a^{m-1},-t_{1} t_{3} / q,-t_{1} t_{4} / q\right)_{\infty}}{\left(t_{1} t_{2} t_{3} t_{4} q^{m-3}\right)_{\infty}} \\
& \times{ }_{3} \phi_{2}\left(\begin{array}{c}
q^{-n},-t_{1} t_{2} q^{n-2}, t_{1} t_{2} t_{3} t_{4} q^{m-3} \\
t_{1} t_{2} t_{3} t_{4} q^{-3},-t_{1} t_{2} q^{m-1}
\end{array} \mid q, q\right) .
\end{aligned}
$$

The $q$-analog of the Pfaff-Saalschütz theorem is [25, (II.12)],

$$
{ }_{3} \phi_{2}\left(\begin{array}{c}
q^{-n}, a, b \\
c, a b c q^{1-n} / c
\end{array} \mid q, q\right)=\frac{(c / a, c / b)_{n}}{(c, c / a b)_{n}} .
$$

Therefore $J_{m, n}$ can be summed. The answer is

$$
\begin{aligned}
J_{m, n}= & \frac{\left(-t_{1} t_{3} / q,-t_{1} t_{4} / q,-t_{3} t_{4} / q,-t_{1} t_{2} q^{m-1},-t_{2} t_{3} q^{m-1},-t_{2} t_{4} q^{m-1}\right)_{\infty}}{\left(t_{1} t_{2} t_{3} t_{4} q^{m-3}\right)_{\infty}} \\
& \times \frac{\left(-t_{3} t_{4} q^{-n-1}, q^{-m}\right)_{n}}{\left(t_{1} t_{2} t_{3} t_{4} q^{-3},-q^{2-m-n} / t_{1} t_{2}\right)_{n}} .
\end{aligned}
$$

Clearly $J_{m, n}=0$ if $m<n$ and

$$
\begin{aligned}
J_{n, n}= & \frac{\left(-t_{1} t_{3} / q,-t_{1} t_{4} / q,-t_{3} t_{4} / q,-t_{1} t_{2} q^{n-1},-t_{2} t_{3} q^{n-1},-t_{2} t_{4} q^{n-1}\right)}{\left(t_{1} t_{2} t_{3} t_{4} q^{-3}\right)_{\infty}} \\
& \times \frac{\left(-q^{2} / t_{3} t_{4}, q\right)_{n}}{\left(-t_{1} t_{2} q^{n-1}\right)_{n}} q^{n(n-7) / 2}\left(-t_{1} t_{2} t_{3} t_{4}\right)^{n} .
\end{aligned}
$$

Therefore

$$
\begin{array}{r}
\int_{-\infty}^{\infty} \varphi_{m}\left(x ; t_{1}, t_{2}, t_{3}, t_{4}\right) \varphi_{n}\left(x ; t_{2}, t_{1}, t_{3}, t_{4}\right) w(x) d \psi(x) \\
=\frac{\left(-t_{1} t_{2} q^{n-2}, q^{-n},-t_{2} t_{3} / q,-t_{2} t_{4} / q\right)_{n}}{\left(q, t_{1} t_{2} t_{3} t_{4} q^{-3}\right)_{n}} q^{n} J_{n, n} \delta_{m, n},
\end{array}
$$

where

$$
w(x)=w\left(x ; t_{1}, t_{2}, t_{3}, t_{4}\right)=\prod_{j=1}^{4}\left(-t_{j}\left(x+\sqrt{x^{2}+1}\right), t_{j}\left(\sqrt{x^{2}+1}-x\right)\right)_{\infty} .
$$

Using the $J_{n, n}$ we establish the biorthogonality relation (1.21). 
In Section 6 we shall prove that the $h_{n}$ 's are also orthogonal with respect to probability measures $d \psi(x ; \sigma)$ with masses

$$
\begin{aligned}
& \psi\left(x_{n}+0^{+}, \sigma\right)-\psi\left(x_{n}-0^{+}, \sigma\right) \\
& =a^{4 n} q^{n(2 n-1)}\left(1+a^{2} q^{2 n}\right) /\left(-a^{2},-q / a^{2}, q ; q\right)_{\infty}, \quad n=0, \pm 1, \ldots .
\end{aligned}
$$

where, see (6.26) and (6.30),

$$
x_{n}=\frac{1}{2}\left(q^{-n} / a-a q^{n}\right), \quad n=0,1,2, \ldots .
$$

Since the evaluation of $I$ in (3.1) only used the integrability of the integrand with respect to a measure that the $h_{n}$ 's are orthogonal with respect to, we can then replace $d \psi$ in (3.1) and (1.21) with $d \psi$ of (3.14) and (3.15). A straightforward calculation shows that $(3.8)$ is equivalent to

$$
\begin{aligned}
& \frac{\prod_{j=1}^{4}\left(a t_{j},-t_{j} / a\right)_{\infty}}{\left(q,-q a^{2},-q / a^{2}\right)_{\infty}} \\
& \quad \times \sum_{-\infty}^{\infty} \frac{\left(-q a / t_{1},-q a / t_{2},-q a / t_{3},-q a / t_{4} ; q\right)_{n}}{\left(a t_{1}, a t_{2}, a t_{3}, a t_{4} ; q\right)_{n}} \frac{1+a^{2} q^{2 n}}{1+a^{2}}\left(t_{1} t_{2} t_{3} t_{4} q^{-3}\right)^{n} \\
& =\frac{1}{\left(t_{1} t_{2} t_{3} t_{4} q^{-3} ; q\right)_{\infty}} \prod_{1 \leq j<k \leq 4}\left(-t_{j} t_{k} / q ; q\right)_{\infty} .
\end{aligned}
$$

The above identity is precisely the ${ }_{6} \psi_{6}$ summation formula of Bailey, formula (II.33) in [25],

$$
\begin{aligned}
{ }_{6} \psi_{6} & \left(\begin{array}{c}
q a^{1 / 2},-q a^{1 / 2}, b, c, d, e \\
a^{1 / 2},-a^{1 / 2}, a q / b, a q / c, a q / d, a q / e
\end{array} \mid q ; \frac{q a^{2}}{b c d e}\right) \\
& =\frac{(q, a q, q / a, a q / b c, a q / b d, a q / b e, a q / c d, a q / c e, a q / d e)_{\infty}}{\left(a q / b, a q / c, a q / d, a q / e, q / b, q / c, q / d, q / e, q a^{2} / b c d e\right)_{\infty}} .
\end{aligned}
$$

The ${ }_{r} \psi_{r}$ function is

$$
{ }_{r} \psi_{r}\left(\begin{array}{c}
a_{1}, \ldots, a_{r} \\
b_{1}, \ldots, b_{r}
\end{array} \mid q, z\right)=\sum_{-\infty}^{\infty} z^{n} \prod_{j=1}^{r} \frac{\left(a_{j} ; q\right)_{n}}{\left(b_{j} ; q\right)_{n}}
$$

with the shifted factorial defined for all $n$ by

$$
(a ; q)_{n}=(a ; q)_{\infty} /\left(a q^{n} ; q\right)_{\infty}
$$




\section{SYMMETRIES AND ASYMPTOTICS}

Recall the Sears transformation for a terminating balanced ${ }_{4} \phi_{3}$ [25], formula (III.15),

$$
\begin{aligned}
{ }_{4} \phi_{3}\left(\begin{array}{c|c}
q^{-n}, A, B, C \\
D, E, F
\end{array}\right. & q, q) \\
& =\frac{(E / A, F / A)_{n}}{(E, F)_{n}} A^{n}{ }_{4} \phi_{3}\left(\begin{array}{c}
q^{-n}, A, D / B, D / C \\
D, A q^{1-n} / E, A q^{1-n} / F
\end{array}\right.
\end{aligned}
$$

provided that $A B C q^{1-n}=D E F$. We now apply the transformation (4.1) to (3.10) with $A=-t_{1} t_{2} q^{n-2}$ and $D=t_{1} t_{2} t_{3} t_{4} q^{-3}$ and find the alternate representation

$$
\begin{aligned}
\varphi_{n}\left(\sinh \xi ; t_{1}, t_{2}, t_{3}, t_{4}\right)= & \frac{\left(-q^{2-n} e^{-\xi} / t_{2}, q^{2-n} e^{\xi} / t_{2}\right)_{n}}{\left(t_{1} e^{-\xi},-t_{1} e^{\xi}\right)_{n}}\left(-t_{1} t_{2}\right)^{n} q^{n(n-2)} \\
& \times{ }_{4} \phi_{3}\left(\begin{array}{c}
q^{-n},-t_{1} t_{2} q^{n-2},-t_{2} t_{3} q^{-2},-t_{2} t_{4} q^{-2} \\
t_{1} t_{2} t_{3} t_{4} q^{-3}, t_{2} q^{-1} e^{-\xi},-t_{2} q^{-1} e^{\xi}
\end{array} \mid q, q\right) \\
= & \left(-t_{1} t_{2}\right)^{n} q^{n(n-2)} \frac{\left(-q^{2-n} e^{-\xi} / t_{2}, q^{2-n} e^{\xi} / t_{2}\right)_{n}}{\left(t_{1} e^{-\xi},-t_{1} e^{\xi}\right)_{n}} \\
& \times \varphi_{n}\left(\sinh \xi ; t_{2} q^{-1}, t_{1} q, t_{3}, t_{4}\right) .
\end{aligned}
$$

In terms of the functions

$$
\psi_{n}\left(\sinh \xi ; t_{1}, t_{2}, t_{3}, t_{4}\right):=t_{1}^{-n}\left(t_{1} e^{-\xi},-t_{1} e^{\xi}\right)_{n} \varphi_{n}\left(\sinh \xi ; t_{1}, t_{2}, t_{3}, t_{4}\right)
$$

the above symmetry relation is

$$
\psi_{n}\left(\sinh \xi ; t_{1}, t_{2}, t_{3}, t_{4}\right)=\psi_{n}\left(\sinh \xi ; t_{2} / q, t_{1} q, t_{3}, t_{4}\right)
$$

Ismail and Wilson [32] established the generating function

$$
\begin{aligned}
& \sum_{n=0}^{\infty} \frac{(a c, a d)_{n}}{(c d, q)_{n}} t^{n} p_{n}(x ; a, b, c, d) \\
& \quad={ }_{2} \phi_{1}\left(\begin{array}{c}
a / z, b / z \\
a b
\end{array} \mid q, a z t\right){ }_{2} \phi_{1}\left(\begin{array}{c}
c z, d z \\
c d
\end{array} \mid q, a t / z\right) .
\end{aligned}
$$

Now choose the parameters $a, b, c, d$ in (4.4) as

$$
\begin{gathered}
a=t_{1} q^{-1} \sqrt{t_{3} t_{4}}, \quad b=q^{-2} t_{2} \sqrt{t_{3} t_{4}}, \quad c=e^{-\xi} q / \sqrt{t_{3} t_{4}}, \\
d=-e^{\xi} q / \sqrt{t_{3} t_{4}}, \quad z=-\sqrt{t_{3} / t_{4}} .
\end{gathered}
$$


This transforms (4.4) to the generating function

$$
\begin{aligned}
\sum_{n=0}^{\infty} & \frac{\left(t_{1} e^{-\xi},-t_{1} e^{\xi}\right)_{n}}{\left(q,-q^{2} / t_{3} t_{4}\right)_{n}} \varphi_{n}\left(\sinh \xi ; t_{1}, t_{2}, t_{3}, t_{4}\right) t^{n} \\
= & { }_{2} \varphi_{1}\left(\begin{array}{c}
-t_{1} t_{4} / q,-t_{2} t_{4} / q^{2} \\
t_{1} t_{2} t_{3} t_{4} q^{-3}
\end{array} \mid q,-t_{1} t_{3} t / q\right) \\
& \times{ }_{2} \phi_{1}\left(\begin{array}{c}
-q e^{-\xi} / t_{4}, q e^{\xi} / t_{4} \\
-q^{2} / t_{3} t_{4}
\end{array} \mid q,-t_{1} t_{4} t / q\right) .
\end{aligned}
$$

Observe that the symmetry relation (4.3) also follows from (4.6).

In [32], Ismail and Wilson derived the asymptotic formula

$$
p_{n}(x ; a, b, c, d) \approx(a / z)^{n} \frac{(a z, b z, c z, d z)_{\infty}}{\left(z^{2}, a b, a c, a d\right)_{\infty}}, \quad|z|<1,
$$

where $z=x-\sqrt{x^{2}-1}, x \notin[-1,1]$. With the parameter identification (4.5), we obtain from (4.7)

$$
\begin{aligned}
& \varphi_{n}\left(\sinh \xi ; t_{1}, t_{2}, t_{3}, t_{4}\right) \\
& \quad \approx\left(-t_{1} t_{4} / q\right)^{n} \frac{\left(-t_{1} t_{3} / q,-t_{2} t_{3} / q^{2},-q e^{-\xi} / t_{4}, q e^{\xi} / t_{4}\right)_{\infty}}{\left(t_{3} / t_{4}, t_{1} t_{2} t_{3} t_{4} q^{-3}, t_{1} e^{-\xi},-t_{1} e^{\xi}\right)_{\infty}},
\end{aligned}
$$

valid for $\left|t_{3}\right|<\left|t_{4}\right|$.

The asymptotics of polynomials orthogonal on a compact set contain a wealth of spectral information on the spectrum and the spectral measures of the polynomials. For example, under certain conditions, see Freud [24], Nevai [38], Szegö [50], Grenander and Szegö [26], a polynomial system $\left\{s_{n}(x)\right\}$ orthonormal on $[-1,1]$ will have the asymptotic development

$$
\begin{array}{ll}
s_{n}(x) \approx z^{-n} / D(z), & x \in \mathbf{C} /[-1,1], \\
z:=x-\sqrt{x^{2}-1}, & \text { as } n \rightarrow \infty .
\end{array}
$$

The weight function $w(x)$ is given by

$$
w(x)=\left(1-x^{2}\right)^{-1 / 2} \lim _{r \rightarrow 1^{-}} D\left(r e^{i \theta}\right) D\left(r e^{-i \theta}\right) .
$$

There is little hope in having results as precise as (4.9) and (4.10) in the case of biorthogonal rational functions. One reason is that the measure that the functions are orthogonal with respect to is not unique. However the asymptotics of biorthogonal rational functions may still provide a glimpse of a measure with respect to which they are biorthogonal. One good example to illustrate this point is the biorthogonal rational functions $\left\{R_{n}(x ; \alpha, \beta, \gamma, \delta ; q)\right\}$ of Al-Salam and Verma [6],

$$
R_{n}(x ; \alpha, \beta, \gamma, \delta ; q)={ }_{3} \phi_{2}\left(\begin{array}{c|c}
\beta, \alpha \gamma / \delta, q^{-n} & q, q) . \\
\beta \gamma / q, \alpha q x & q
\end{array}\right) .
$$


Al-Salam and Verma [6] proved the biorthogonality relation

$$
\int_{-\infty}^{\infty} w(x) R_{n}(x ; \alpha, \beta, \gamma, \delta ; q) R_{m}(x ; \delta, \gamma, \beta, \alpha ; q) d_{q} x=K \frac{(\beta \gamma / q)^{n}}{(\beta \gamma / q)_{n}} \delta_{m, n},
$$

where $K$ is a certain constant [28], p. 226, and

$$
w(x)=(\alpha x q, \delta x q)_{\infty} /(q \alpha x / \beta, q \delta x / \gamma)_{\infty} .
$$

The integral in (4.12) is the bilateral $q$-integral

$$
\int_{-\infty}^{\infty} f(x) d_{q} x:=(1-q) \sum_{-\infty}^{\infty} q^{n}\left[f\left(q^{n}\right)+f\left(-q^{n}\right)\right] .
$$

The form of $w(x)$ given in [6] contains obvious misprints corrected in (4.13). We shall first determine the asymptotic behavior of the $R_{n}$ 's and indicate how the biorthogonality relation (4.12) could have been suggested by the asymptotics of the $R_{n}$ 's.

To determine the large $n$ behavior of $R_{n}$ we identify $R_{n}$ as a special AskeyWilson polynomial. Choose

$$
\begin{aligned}
& a=\sqrt{\alpha \beta \gamma / \delta}, \quad z=\sqrt{\beta \delta / \alpha \gamma}, \quad b=0, \\
& c=q^{-1} \sqrt{\beta \gamma \delta / \alpha}, \quad d=q x \sqrt{\alpha \delta / \beta \gamma},
\end{aligned}
$$

in (1.3). Now (4.7) yields

$$
R_{n}(x ; \alpha, \beta, \gamma, \delta ; q) \approx\left(\frac{\alpha \gamma}{\delta}\right)^{n}\left(\beta, \frac{\beta \delta}{\alpha q}, q x \frac{\delta}{\gamma}\right)_{\infty} /\left(\frac{\beta \delta}{\alpha \gamma}, \frac{\beta \gamma}{q}, \alpha x q\right)_{\infty},
$$

as $n \rightarrow \infty$, provided that $|\beta \delta|<|\alpha \gamma|$ (that is $|z|<1$ ). If $|\beta \delta|>|\alpha \gamma|$ we replace $z$ in (4.15) by $\sqrt{\alpha \gamma / \beta \delta}$ and obtain

$$
R_{n}(x ; \alpha, \beta, \gamma, \delta ; q) \approx \beta^{n}\left(\frac{\alpha \gamma}{\beta}, \frac{\gamma}{q}, q x \frac{\alpha}{\beta}\right)_{\infty} /\left(\frac{\alpha \gamma}{\beta \delta}, \frac{\beta \gamma}{q}, \alpha x q\right)_{\infty},
$$

as $n \rightarrow \infty$ if $|\beta \delta|>|\alpha \gamma|$.

It is clear that the asymptotic relations (4.16) and (4.17) suggest the weight function (4.13).

We now consider the functions $\left\{\varphi_{n}\right\}$. The asymptotic formula (4.8) suggests the weight function

$$
\begin{aligned}
\omega(\sinh \xi) & =\omega\left(\sinh \xi ; t_{1}, t_{2}, t_{3}, t_{4}\right) \\
& :=\frac{\left(t_{1} e^{-\xi},-t_{1} e^{\xi}, t_{2} e^{-\xi},-t_{2} e^{\xi}\right)_{\infty}}{\left(q e^{\xi} / t_{3},-q e^{-\xi} / t_{3}, q e^{\xi} / t_{4},-q e^{-\xi} / t_{4}\right)_{\infty}} .
\end{aligned}
$$

With proper interpretation this does turn out to be a weight function. Since the corresponding measure is discrete it must be related to $\omega(x) d x$. Let $\alpha(x)$ be 
a jump function with jumps

$$
\alpha\left(x_{n}(a)+0^{+}\right)-\alpha\left(x_{n}(a)-0^{+}\right)=2 a \frac{\cosh \left(\xi_{n}(a)\right)}{\left(q,-q a^{2},-q / a^{2}\right)_{\infty}},
$$

at $x=x_{n}(a)$ where

$$
x_{n}(a)=\frac{1}{2}\left(q^{-n} / a-a q^{n}\right), \quad x_{n}(a)=\sinh \left(\xi_{n}(a)\right) .
$$

The $x_{n}(a)$ 's are the same as the spectral points of the $q^{-1}$-Hermite polynomials, see (6.27). The term $\cosh \left(\xi_{n}(a)\right)$ is a constant multiple of $\frac{d x_{n}(a)}{d a}$.

Before we prove the biorthogonality of the $\varphi_{n}$ 's we need to evaluate the total $\omega$ mass.

Theorem 4.1. We have the evaluation

$$
J\left(t_{1}, t_{2}, t_{3}, t_{4}\right):=\int_{-\infty}^{\infty} \omega(x) d \alpha(x)=\frac{\prod_{1 \leq j<k \leq 4}\left(-t_{j} t_{k} / q\right)_{\infty}}{\left(t_{1} t_{2} t_{3} t_{4} / q^{3}\right)_{\infty} \prod_{j=1}^{4}\left(a t_{j},-t_{j} / a\right)_{\infty}} .
$$

Proof. It is clear from (4.20) that $e^{\xi_{n}(a)}=q^{-n} / a$. Hence

$$
\begin{aligned}
\left(q,-q a^{2},-q / a^{2}\right)_{\infty} J\left(t_{1}, t_{2}, t_{3}, t_{4}\right) & \\
= & \sum_{-\infty}^{\infty} \frac{\left(a^{2} q^{n}+q^{-n}\right)\left(a q^{n} t_{1},-t_{1} q^{-n} / a, a q^{n} t_{2},-t_{2} q^{-n} / a\right)_{\infty}}{\left(q^{1-n} / a t_{3},-a q^{n+1} / t_{3}, q^{1-n} / a t_{4},-a q^{n+1} / t_{4}\right)_{\infty}} \\
= & \frac{\left(a t_{1},-t_{1} / a, a t_{2},-t_{2} / a\right)_{\infty}}{\left(q / a t_{3},-a q / t_{3}, q / a t_{4},-a q / t_{4}\right)_{\infty}} \\
& \quad \times{ }_{6} \psi_{6}\left(\begin{array}{c}
i q a,-i q a,-a q / t_{1},-a q / t_{2},-a q / t_{3},-a q / t_{4} \\
i a,-i a, a t_{1}, a t_{2}, a t_{3}, a t_{4}
\end{array} \mid q ; \frac{t_{1} t_{2} t_{3} t_{4}}{q^{3}}\right) .
\end{aligned}
$$

We now apply the ${ }_{6} \psi_{6}$ sum (3.17) and establish (4.21).

Theorem 4.2. The rational functions $\left\{\varphi_{n}\right\}$ satisfy the biorthogonality relation

$$
\int_{-\infty}^{\infty} \varphi_{m}\left(x ; t_{1}, t_{2}, t_{3}, t_{4}\right) \varphi_{n}\left(x ; t_{2}, t_{1}, t_{3}, t_{4}\right) \omega(x) d \alpha(x)=g_{n} \delta_{m, n},
$$

where $\omega(x), \alpha$, and $g_{n}$ are as in (4.18), (4.19)-(4.20), and (1.22), respectively. Proof. As we did in Section 3, consider the integrals

$$
J_{m, n}:=\int_{-\infty}^{\infty} \frac{\varphi_{n}\left(\sinh \xi ; t_{1}, t_{2}, t_{3}, t_{4}\right)}{\left(-t_{2} e^{\xi},-t_{2} e^{-\xi}\right)_{m}} \omega(\sinh \xi) d \alpha(\xi) .
$$

From this, (1.22), and (4.20) it follows that

$$
\begin{aligned}
J_{m, n}= & \sum_{k=0}^{n} \frac{\left(q^{-n},-t_{1} t_{2} q^{n-2},-t_{1} t_{3} / q,-t_{1} t_{4} / q\right)_{k}}{\left(q, t_{1} t_{2} t_{3} t_{4} q^{-3}\right)_{k}} q^{k} J\left(t_{1} q^{k}, t_{2} q^{m}, t_{3}, t_{4}\right) \\
= & \frac{\left(-t_{3} t_{4} / q,-t_{2} t_{3} q^{m-1},-t_{2} t_{4} q^{m-1},-t_{1} t_{2} a^{m-1},-t_{1} t_{3} / q,-t_{1} t_{4} / q\right)_{\infty}}{\left(t_{1} t_{2} t_{3} t_{4} q^{m-3}\right)_{\infty}} \\
& \times{ }_{3} \phi_{2}\left(\begin{array}{c}
q^{-n},-t_{1} t_{2} q^{n-2}, t_{1} t_{2} t_{3} t_{4} q^{m-3} \\
t_{1} t_{2} t_{3} t_{4} q^{-3},-t_{1} t_{2} q^{m-1}
\end{array}\right.
\end{aligned}
$$


Again we sum the ${ }_{3} \phi_{2}$ by the $q$-analog of the Pfaff-Saalschütz theorem (3.12). The rest of the proof parallels the proof of Theorem 3.2 and will be omitted.

Al-Salam and Ismail [5] proved that the rational functions $R_{n}$ and $S_{n}$,

$$
R_{n}\left(z ; a, b, t_{1}, t_{2}\right):={ }_{4} \phi_{3}\left(\begin{array}{c|c}
q^{-n}, q^{-1 / 2} t_{1} z, t_{1}, a b t_{1} t_{2} q^{n-1} & q, q), \\
b q^{1 / 2} t_{1} z, a t_{1}, t_{1} t_{2} / q & q
\end{array}\right)
$$

$$
S_{n}\left(z ; a, b, t_{1}, t_{2}\right):=R_{n}\left(z ; b, a, t_{2}, t_{1}\right)
$$

satisfy the biorthogonality relation

$$
\frac{1}{2 \pi} \int_{|z|=1} K(z) R_{n}(z) \overline{S_{m}(z)} \frac{d z}{z}=\frac{\left(a b q, a b t_{1} t_{2} q^{n-1}, q\right)_{n}}{\left(a b t_{1} t_{2}\right)_{2 n}\left(t_{1} t_{2} q^{-1}\right)_{n}}\left(t_{1} t_{2} q^{-1}\right)^{n} \delta_{n, m}
$$

where the weight function $K(z)$ is

$$
K(z)=\frac{\left(q^{1 / 2} z, q^{1 / 2} / z, a q^{1 / 2} t_{2} z, b q^{1 / 2} t_{1} / z, a t_{1}, b t_{2}, q, a b q, t_{1} t_{2} / q ; q\right)_{\infty}}{\left(a q^{1 / 2} z, b q^{1 / 2} / z, q^{-1 / 2} t_{2} z, q^{-1 / 2} t_{1} / z, t_{1}, t_{2}, a q, b q, a b t_{1} t_{2} ; q\right)_{\infty}} .
$$

If $r_{n}(z)$ and $s_{n}(z)$ denote the biorthonormal functions then, as was observed in [5], (4.7) shows that $z^{-n} r_{n}(z)$ and $z^{-n} s_{n}(z)$ converge uniformly on compact subsets of $\left\{z:|z|>q^{1 / 2}\right\}$ to $\rho(z)$ and $\sigma(z)$, respectively. Furthermore

$$
\lim _{r \rightarrow 1} \rho\left(r e^{i \theta}\right) \overline{\sigma\left(r e^{i \theta}\right)}=1 / K\left(e^{i \theta}\right) .
$$

This is the exact analog of (4.9) and (4.10).

\section{COMPUTATION OF ENTIRE FUNCTIONS}

Following the notation in [47] we consider polynomial solutions of a three term recurrence relation

$$
\omega_{n+1}(z)=\left(z-\alpha_{n+1}\right) \omega_{n}(z)-\beta_{n} \omega_{n-1}(z), \quad n>0 .
$$

Two linear independent solutions $\left\{P_{n}(z)\right\}$ and $\left\{Q_{n}(z)\right\}$ can be generated using (5.1) from the initial conditions

$$
Q_{0}(z)=1, \quad Q_{1}(z)=z-\alpha_{1}, \quad P_{0}(z)=0, \quad P_{1}(z)=\beta_{0} .
$$

It is assumed that

$$
\alpha_{n} \text { is real, } \quad n>0, \beta_{n}>0, n \geq 0 .
$$

Starting with the polynomials $\left\{Q_{n}(z)\right\}$ such that (5.3) holds we can define a positive linear functional $L$ on the space of polynomials by $L\left(Q_{n}\right)=\delta_{n, 0}$. The functional $L$ can be represented as an integral with respect to a positive measure, normalized to have a total mass 1 , since $Q_{0}=1$. This measure will be unique if and only if the corresponding moment problem has a unique solution, that is when we have a determinate moment problem. In the indeterminate case the totality of probability measures $\{d \psi(t ; \sigma)\}$ that solve the moment problem can be indexed by functions $\sigma(z)$ analytic in the upper half plane $\Im z>0$ and 
satisfying $\Im \sigma(z) \leq 0$ for $\Im z>0$. Furthermore, there exist entire functions $A(z), B(z), C(z), D(z)$, such that

$$
\frac{A(z)-\sigma(z) C(z)}{B(z)-\sigma(z) D(z)}=\int_{-\infty}^{\infty} \frac{d \psi(t ; \sigma)}{z-t} .
$$

In fact $A(z), B(z), C(z), D(z)$ are uniform limits, on compact subsets of C, of $A_{n}(z), B_{n}(z), C_{n}(z), D_{n}(z)$, respectively, where

$$
\begin{aligned}
& A_{n+1}(z)=\left[P_{n+1}(z) P_{n}(0)-P_{n+1}(0) P_{n}(z)\right]\left(\beta_{0} \beta_{1} \cdots \beta_{n}\right)^{-1}, \\
& B_{n+1}(z)=\left[Q_{n+1}(z) P_{n}(0)-P_{n+1}(0) Q_{n}(z)\right]\left(\beta_{0} \beta_{1} \cdots \beta_{n}\right)^{-1}, \\
& C_{n+1}(z)=\left[P_{n+1}(z) Q_{n}(0)-Q_{n+1}(0) P_{n}(z)\right]\left(\beta_{0} \beta_{1} \cdots \beta_{n}\right)^{-1}, \\
& D_{n+1}(z)=\left[Q_{n+1}(z) Q_{n}(0)-Q_{n+1}(0) Q_{n}(z)\right]\left(\beta_{0} \beta_{1} \cdots \beta_{n}\right)^{-1} .
\end{aligned}
$$

The purpose of this section is to construct the entire functions $A(z)$, $B(z), C(z)$ and $D(z)$ explicitly in the case of the $q^{-1}$-Hermite polynomials $\left\{h_{n}(x \mid q)\right\}$. This is achieved by determining the large $n$ behavior of $P_{n}(z)$ and $Q_{n}(z)$ and then use it to compute the limits of $A_{n}(z), B_{n}(z), C_{n}(z)$, and $D_{n}(z)$.

We first need to put the recurrence relation in (1.16) in monic form. Set

$$
Q_{n}(x)=2^{-n} h_{n}(x \mid q) \text {. }
$$

The $Q_{n}$ 's satisfy (5.2) and (5.3) with

$$
\alpha_{n}=0, \quad \beta_{n}=\frac{1}{4} q^{-n}\left(1-q^{n}\right), \quad n>0, \quad 0<q<1, \quad \beta_{0}=1 .
$$

It is easy to see that when $\alpha_{n}=0$ for all $n$ then (5.1) and (5.2) imply

$$
Q_{2 n}(0)=(-1)^{n} \beta_{1} \beta_{3} \cdots \beta_{2 n-1}, \quad P_{2 n+1}(0)=(-1)^{n} \beta_{0} \beta_{2} \cdots \beta_{2 n} .
$$

Hence

$$
Q_{2 n+1}(0)=0, \quad P_{2 n}(0)=0,
$$

In the case of $\left\{h_{n}(x \mid q)\right\}$ we have, in view of (5.9),

$$
\begin{aligned}
& A_{2 n}(z)=\frac{(-1)^{n-1} 4^{n} q^{n^{2}}}{\left(q ; q^{2}\right)_{n}} P_{2 n}(z), C_{2 n}(z)=\frac{(-1)^{n-1} 4^{n-1} q^{n(n-1)}}{\left(q^{2} ; q^{2}\right)_{n-1}} P_{2 n-1}(z) \\
& B_{2 n}(z)=\frac{(-1)^{n-1} q^{n^{2}}}{\left(q ; q^{2}\right)_{n}} h_{2 n}(z \mid q), D_{2 n}(z)=\frac{(-1)^{n-1} q^{n(n-1)}}{2\left(q^{2} ; q^{2}\right)_{n-1}} h_{2 n-1}(z \mid q)
\end{aligned}
$$

We now compute the strong asymptotics of $\left\{h_{n}(x \mid q)\right\}$ using Darboux's method, [50], [39]. The application of the method of Darboux requires a generating function having singularities in the finite complex plane. The generating 
function (2.4) is entire so we need to find a generating function suitable for the application of Darboux's method. To do so, set

$$
h_{n}(x \mid q)=q^{-n^{2} / 4}(\sqrt{q} ; \sqrt{q})_{n} s_{n}(x) .
$$

In terms of the $s_{n}$ 's, the recurrence relation in (1.16) becomes

$$
\left(1-q^{(n+1) / 2}\right) s_{n+1}(x)=2 x q^{(n+1 / 2) / 2} s_{n}(x)-\left(1+q^{n / 2}\right) s_{n-1}(x) .
$$

Therefore, the generating function

$$
G(x, t):=\sum_{0}^{\infty} s_{n}(x) t^{n}
$$

transforms $(5.20)$ to the $q$-difference equation

$$
G(x, t)=\frac{1+2 x q^{1 / 4} t-t^{2} q^{1 / 2}}{1+t^{2}} G(x, \sqrt{q} t) .
$$

By iterating the above functional equation we find

$$
G(x, t)=\frac{(t \alpha, t \beta ; \sqrt{q})_{n}}{\left(-t^{2} ; q\right)_{n}} G\left(x, q^{n / 2} t\right) .
$$

Since $G(x, t) \rightarrow 1$ as $t \rightarrow 0$ we let $n \rightarrow \infty$ in the above functional equation. This establishes the generating function

$$
\sum_{0}^{\infty} s_{n}(x) t^{n}=\frac{(t \alpha, t \beta ; \sqrt{q})_{\infty}}{\left(-t^{2} ; q\right)_{\infty}}
$$

where

$$
\alpha=-\left(x+\sqrt{x^{2}+1}\right) q^{1 / 4}=-q^{1 / 4} e^{\xi}, \quad \beta=\left(\sqrt{x^{2}+1}-x\right) q^{1 / 4}=q^{1 / 4} e^{-\xi} .
$$

The $t$ singularities with smallest absolute value of the right side of (5.21) are $t= \pm i$. Thus Darboux's method gives

$$
s_{n}(x) \approx \frac{(i \alpha, i \beta ; \sqrt{q})_{\infty}}{2(q ; q)_{\infty}}(-i)^{n}+\frac{(-i \alpha,-i \beta ; \sqrt{q})_{\infty}}{2(q ; q)_{\infty}} i^{n} .
$$

Therefore

$$
\begin{gathered}
h_{2 n}(x \mid q) \approx q^{-n^{2}} \frac{(\sqrt{q} ; \sqrt{q})_{\infty}}{2(q ; q)_{\infty}}(-1)^{n}\left[(i \alpha, i \beta ; \sqrt{q})_{\infty}+(-i \alpha,-i \beta ; \sqrt{q})_{\infty}\right], \\
h_{2 n+1}(x \mid q) \approx i q^{-n^{2}-n-1 / 4} \frac{(\sqrt{q} ; \sqrt{q})_{\infty}}{2(q ; q)_{\infty}}(-1)^{n+1} \\
\quad \times\left[(i \alpha, i \beta ; \sqrt{q})_{\infty}-(-i \alpha,-i \beta ; \sqrt{q})_{\infty}\right] .
\end{gathered}
$$

It is now clear that the functions $B(z)$ and $D(z)$ can be found from (5.18) and (5.25). This provides a representation of $B(z)$ and $D(z)$ as sums of two terms. The two term sums in $B(z)$ and $D(z)$ can be simplified to single terms using quartic transformations. Next we shall give direct proofs of this simplification and discuss the connection with quartic transformations at the end of this section. 
Observe that the three term recurrence relation in (1.16) implies

$$
\begin{aligned}
4 x^{2} h_{n}(x \mid q)= & h_{n+2}(x \mid q)+\left[q^{-n-1}(1+q)-2\right] h_{n}(x \mid q) \\
& +q^{1-2 n}\left(1-q^{n}\right)\left(1-q^{n-1}\right) h_{n-2}(x \mid q) .
\end{aligned}
$$

Askey and Ismail [16] studied the polynomials $\left\{v_{n}(z ; p ; a, b, c)\right\}$, or simply $\left\{v_{n}\right\}$, generated by

$$
v_{0}=1, \quad v_{1}=(a-z) /(1-p),
$$

$$
\left(1-p^{n+1}\right) v_{n+1}=\left(a-z p^{n}\right) v_{n}-\left(b-c p^{n-1}\right) v_{n-1}, \quad n>0, \quad 0<p<1 .
$$

The $v_{n}$ 's are Al-Salam-Chihara polynomials when $q>1$ [22]. It is easy to see from (5.26), (5.27), and (5.28) that the polynomials $\left\{r_{n}(y)\right\}$,

$$
r_{n}(y):=h_{2 n}(x \mid q) q^{n(n-1 / 2)}(-1)^{n} /\left(q^{2} ; q^{2}\right)_{n}, \quad y:=\left(4 x^{2}+2\right) \sqrt{q},
$$

satisfy (5.28) with $z=y, p=q^{2}, b=1, c=q, a=(1+q) / \sqrt{q}$. Askey and Ismail [16] established the generating function

$$
\sum_{0}^{\infty} v_{n}(z ; p ; a, b, c) t^{n}=\prod_{n=0}^{\infty}\left[\frac{1-z t p^{n}+c t^{2} p^{2 n}}{1-a t p^{n}+b t^{2} p^{2 n}}\right] .
$$

Therefore we obtain the generating function

$$
\sum_{n=0}^{\infty} \frac{h_{2 n}(x \mid q)}{\left(q^{2} ; q^{2}\right)_{n}}(-t)^{n} q^{n(n-1 / 2)}=\prod_{n=0}^{\infty}\left[\frac{1-\left(4 x^{2}+2\right) t q^{2 n+1 / 2}+t^{2} q^{4 n+1}}{1-(1+q) t q^{2 n-1 / 2}+t^{2} q^{4 n}}\right] .
$$

Applying Darboux's method to (5.30) we establish the alternate asymptotic relationship

$$
h_{2 n}(x \mid q) \approx \frac{(-1)^{n} q^{-n^{2}}}{\left(q ; q^{2}\right)_{\infty}} \prod_{n=0}^{\infty}\left[1-\left(4 x^{2}+2\right) q^{2 n+1}+q^{4 n+2}\right] .
$$

This yields

$$
\begin{aligned}
B(x) & =-\left(q ; q^{2}\right)_{\infty}^{-2} \prod_{n=0}^{\infty}\left[1-\left(4 x^{2}+2\right) q^{2 n+1}+q^{4 n+2}\right] \\
& =-\left(q ; q^{2}\right)_{\infty}^{-2}\left(q e^{2 \xi}, q e^{-2 \xi} ; q^{2}\right)_{\infty} .
\end{aligned}
$$

Similarly we can use $(5.26),(5.27)$ and (5.28) together with (1.16) to prove that

$$
v_{n}\left(q^{3 / 2}\left(4 x^{2}+2\right) ; q^{2} ; q^{1 / 2}+q^{-1 / 2}, 1, q^{3}\right)=\frac{(-1)^{n} q^{n(n+1 / 2)}}{(2 x)\left(q^{2} ; q^{2}\right)_{n}} h_{2 n+1}(x \mid q) .
$$

Now (5.29) gives

$$
\sum_{n=0}^{\infty} \frac{h_{2 n+1}(x \mid q)}{\left(q^{2} ; q^{2}\right)_{n}}(-t)^{n} q^{n(n+1 / 2)}=2 x \prod_{n=0}^{\infty} \frac{1-\left(4 x^{2}+2\right) t q^{2 n+3 / 2}+t^{2} q^{4 n+3}}{1-(1+q) t q^{2 n-1 / 2}+t^{2} q^{4 n}} .
$$


Finally we apply Darboux's method to (5.34) and derive the asymptotic formula

$$
h_{2 n+1}(x \mid q) \approx \frac{(-1)^{n} q^{-n(n+1)} 2 x}{\left(q ; q^{2}\right)_{\infty}} \prod_{n=0}^{\infty}\left[1-\left(4 x^{2}+2\right) q^{2 n+2}+q^{4 n+4}\right] .
$$

Formula (5.35) is just what we need to combine with (5.18) in order to find $D(z)$. The answer is

$$
\begin{aligned}
D(x) & =\frac{x}{(q ; q)_{\infty}} \prod_{n=0}^{\infty}\left[1-\left(4 x^{2}+2\right) q^{2 n+2}+q^{4 n+4}\right] \\
& =\frac{x}{(q ; q)_{\infty}}\left(q^{2} e^{2 \xi}, q^{2} e^{-2 \xi} ; q^{2}\right)_{\infty} .
\end{aligned}
$$

Note that the orthonormal polynomials are $\left\{h_{n}(x \mid q) q^{n(n+1) / 4} / \sqrt{(q ; q)_{n}}\right\}$. From (5.31) and (5.35) it is now clear that the sum of squares of absolute values of the orthonormal $h_{n}$ 's converges for every $x$ in the complex plane. This confirms the indeterminacy of the moment problem, [47], Corollary 2.7, p. 50. Observe that (5.25) and (5.35) lead to the identity

$$
\begin{gathered}
(\sqrt{q} ; q)_{\infty}\left[\left(i q^{1 / 4} e^{\xi},-i q^{1 / 4} e^{-\xi} ; \sqrt{q}\right)_{\infty}+\left(-i q^{1 / 4} q^{\xi}, i q^{1 / 4} e^{-\xi} ; \sqrt{q}\right)_{\infty}\right] \\
=2\left(q e^{2 \xi}, q e^{-2 \xi} ; q^{2}\right)_{\infty} /\left(q ; q^{2}\right) .
\end{gathered}
$$

Similarly (5.24) and (5.25) imply

$$
\begin{gathered}
(\sqrt{q} ; q)_{\infty}\left[\left(i q^{1 / 4} e^{\xi},-i q^{1 / 4} e^{-\xi} ; \sqrt{q}\right)_{\infty}-\left(-i q^{1 / 4} e^{\xi}, i q^{1 / 4} e^{-\xi} ; \sqrt{q}\right)_{\infty}\right] \\
=-4 i q^{1 / 4} \sinh \xi\left(q^{2} e^{2 \xi}, q^{2} e^{-2 \xi} ; q^{2}\right)_{\infty} /\left(q ; q^{2}\right)_{\infty}
\end{gathered}
$$

The identities (5.37) and (5.38) give explicitly the real and imaginary parts of the function $\left(i q^{1 / 4} e^{\xi},-i q^{1 / 4} e^{-\xi} ; \sqrt{q}\right)_{\infty}$ and are instances of quartic transformations. When (5.37) and (5.38) are expressed in terms of theta functions, see (6.20) and (6.21), they give the formulas in Example 1, p. 464 in [52].

Next we determine the large $n$ asymptotics of $P_{n}(x)$. Following the notation in [16], the numerator polynomials $\left\{h_{n}^{*}(x \mid q)\right\}$ satisfies the difference equation in (1.16) and the initial conditions

$$
h_{0}^{*}(x \mid q)=0, \quad h_{1}^{*}(x \mid q)=2 .
$$

We then have

$$
P_{n}(x)=2^{-n} h_{n}^{*}(x \mid q) .
$$

In order to find a generating function for $\left\{h_{n}^{*}(x \mid q)\right\}$ we mimic the renormalization (5.19) and let

$$
h_{n}^{*}(x \mid q)=q^{-n^{2} / 4}(\sqrt{q} ; \sqrt{q})_{n} s_{n}^{*}(x) .
$$


The $s_{n}^{*}$ 's also satisfy $(5.20)$ but $s_{0}^{*}(x)=0, s_{1}^{*}(x)=2 q^{1 / 4} /(1-\sqrt{q})$. The generating function

$$
G^{*}(x, t)=\sum_{0}^{\infty} s_{n}^{*}(x) t^{n}
$$

transforms the recurrence relation (5.20) to

$$
G^{*}(x, t)=\frac{1+2 x q^{1 / 4} t-t^{2} q^{1 / 2}}{1+t^{2}} G^{*}(x, \sqrt{q} t)+\frac{2 q^{1 / 4} t}{1+t^{2}} .
$$

The solution to the above $q$-difference equation with $G^{*}(x, 0)=0$,

$$
\left.\frac{\partial G^{*}}{\partial t}(x, t)\right|_{t=0}=s_{1}^{*}(x)
$$

is

$$
\sum_{0}^{\infty} s_{n}^{*}(x) t^{n}=2 q^{1 / 4} t \sum_{n=0}^{\infty} \frac{(t \alpha, t \beta ; \sqrt{q})_{n}}{\left(-t^{2} ; q\right)_{n+1}} q^{n / 2} .
$$

Now Darboux's method gives

$$
\begin{aligned}
h_{n}^{*}(x \mid q) \approx & -q^{\left(1-n^{2}\right) / 4}(\sqrt{q} ; \sqrt{q})_{\infty} i^{n+1} \\
& \times\left[{ }_{2} \phi_{1}(-i \alpha \sqrt{q},-i \beta \sqrt{q} ;-\sqrt{q} ; \sqrt{q}, \sqrt{q})\right. \\
& \left.+(-1)^{n+1}{ }_{2} \phi_{1}(i \alpha \sqrt{q}, i \beta \sqrt{q} ;-\sqrt{q} ; \sqrt{q}, \sqrt{q})\right] .
\end{aligned}
$$

In order to simplify the right side we need to go back to the recurrence relation in (1.16) and obtain separate generating functions for $\left\{h_{2 n}^{*}(x \mid q)\right\}$ and $\left\{h_{2 n+1}^{*}(x \mid q)\right\}$ as we did for the $h_{n}$ 's. Then $h_{n}^{*}$ 's will satisfy (5.26). The $r_{n}$ 's defined above (5.29) satisfy

$$
\left(1-q^{2 n+2}\right) r_{n+1}(y)+\left(1-q^{2 n-1}\right) r_{n}(y)+\left(y q^{2 n}-(1+q) / \sqrt{q}\right) r_{n}(x)=0 .
$$

The polynomial $\left\{r_{n}^{*}(y)\right\}$ satisfies the same recurrence relation but $r_{0}^{*}(y)=0$, and $r_{1}^{*}(y)$ is the coefficient of $y$ in $r_{1}(y)$. This is the notation of [16]. Thus $r_{1}^{*}(y)=-1 /\left(1-q^{2}\right)$. It is now easy to see that

$$
r_{n}^{*}(y)=\frac{q^{n(n-1 / 2)}(-1)^{n} q^{-1 / 2}}{4 x\left(q^{2} ; q^{2}\right)_{n}} h_{2 n}^{*}(x \mid q), \quad y:=\left(4 x^{2}+2\right) \sqrt{q} .
$$

Therefore

$$
h_{2 n}^{*}(x \mid q)=4 x\left(q^{2} ; q^{2}\right)_{n} q^{1 / 2}(-1)^{n} q^{-n(n-1 / 2)} v_{n}^{*}\left(y ; q^{2},(1+q) / \sqrt{q}, 1, q\right) .
$$

Askey and Ismail [16] established the generating function

$$
\sum_{n=0}^{\infty} t^{n} v_{n}^{*}(z ; p ; a, b, c)=\frac{-t}{1-a t+b t^{2}} \sum_{n=0}^{\infty} p^{n} \prod_{j=0}^{n-1} \frac{1-y t p^{j}+c t^{2} p^{2 j}}{1-a t p^{j+1}+b t^{2} p^{2 j+2}},
$$

from which it follows that

$$
h_{2 n}^{*}(x \mid q) \approx 4 x(-1)^{n+1} q^{-n^{2}} \frac{q}{1-q}\left(q^{2} ; q^{2}\right)_{\infty 2} \phi_{1}\left(q e^{2 \xi}, q e^{-2 \xi} ; q^{3} ; q^{2}, q^{2}\right) .
$$


We now come to the asymptotics of $h_{2 n+1}^{*}(x \mid q)$. Set

$$
w_{n}\left(q^{3 / 2}\left(2+4 x^{2}\right)\right)=(-1)^{n} q^{n(n+1 / 2)} h_{2 n+1}^{*}(x \mid q) /\left(q^{3} ; q^{2}\right)_{n} .
$$

Thus (5.39) and (1.16) show that $w_{0}$ and $w_{1}$ are

$$
w_{0}(y)=2, \quad w_{1}(y)=2\left[q^{-1 / 2}\left(1+q^{2}\right)-y\right] /\left(1-q^{3}\right),
$$

with

$$
y:=q^{3 / 2}\left(2+4 x^{2}\right)
$$

Furthermore (5.26) implies

$$
\left(1-q^{2 n+3}\right) w_{n+1}(y)+\left[q^{2 n} y-(1+q) / \sqrt{q}\right] w_{n}(y)+\left(1-q^{2 n}\right) w_{n-1}(y)=0, \quad n>0 .
$$

Set

$$
W(y, t):=\sum_{0}^{\infty} w_{n}(y) t^{n} .
$$

The three term recurrence relation of the $w_{n}$ 's is equivalent to the $q$ difference equation

$$
\begin{aligned}
& W(y, t)\left\{1-t(1+q) / \sqrt{q}+t^{2}\right\}-\left\{q-t y+q^{2} t^{2}\right\} W\left(y, q^{2} t\right) \\
& =(1-q) w_{0}(y)+\left(1-q^{3}\right) t w_{1}(y)+t[y-(1+q) / \sqrt{q}] w_{0}(y) .
\end{aligned}
$$

Therefore, with $x=\sinh \xi, y$ becomes $2 q^{3 / 2} \cosh 2 \xi$ and we have

$$
W(y, t)=\frac{q\left(1-t \sqrt{q} e^{2 \xi}\right)\left(1-t \sqrt{q} e^{-2 \xi}\right)}{(1-t \sqrt{q})(1-t / \sqrt{q})} W\left(y, q^{2} t\right)+\frac{2(1-q)}{(1-t / \sqrt{q})} .
$$

By iteration we obtain

$$
\sum_{n=0}^{\infty} w_{n}(y) t^{n}=\frac{2(1-q)}{1-t / \sqrt{q}}{ }_{3} \phi_{2}\left(t e^{2 \xi} \sqrt{q}, t e^{-2 \xi} \sqrt{q}, q^{2} ; t q^{1 / 2}, t q^{3 / 2} ; q^{2}, q\right) .
$$

This establishes, via Darboux's method, the limiting relation

$$
w_{n}(y) \approx 2(1-q) q^{-n / 2}{ }_{2} \phi_{1}\left(q e^{2 \xi}, q e^{-2 \xi} ; q ; q^{2}, q\right),
$$

which implies

$$
h_{2 n+1}^{*}(x \mid q) \approx 2\left(q ; q^{2}\right)_{\infty}(-1)^{n} q^{-n(n+1)}{ }_{2} \phi_{1}\left(q e^{2 \xi}, q e^{-2 \xi} ; q ; q^{2}, q\right) .
$$

From (5.17), (5.40), and (5.43) we see that

$$
A_{2 n}(x) \approx \frac{4 x q\left(q^{2} ; q^{2}\right)_{\infty}}{(1-q)\left(q ; q^{2}\right)_{\infty}}{ }_{2} \phi_{1}\left(q e^{2 \xi}, q e^{-2 \xi} ; q^{3} ; q^{2}, q^{2}\right) .
$$

Similarly (5.17), (5.40), and (5.44) imply

$$
C_{2 n}(x) \approx \frac{\left(q ; q^{2}\right)_{\infty}}{\left(q^{2} ; q^{2}\right)_{\infty}}{ }_{2} \phi_{1}\left(q e^{2 \xi}, q e^{-2 \xi} ; q ; q^{2}, q\right) .
$$


This evaluates $A(x)$ and $C(x)$ as

$$
\left\{\begin{array}{l}
A(x)=\frac{4 x q\left(q^{2} ; q^{2}\right)_{\infty}}{(1-q)\left(q ; q^{2}\right)_{\infty}}{ }_{2} \phi_{1}\left(q e^{2 \xi}, q e^{-2 \xi} ; q^{3} ; q^{2}, q^{2}\right) \\
C(x)=\frac{\left(q ; q^{2}\right)_{\infty}}{\left(q^{2} ; q^{2}\right)_{\infty}}{ }_{2} \phi_{1}\left(q e^{2 \xi}, q e^{-2 \xi} ; q ; q^{2}, q\right) .
\end{array}\right.
$$

Also by combining (5.42) with (5.43) and (5.44) we discover the quartic transformations

$$
\begin{aligned}
{ }_{2} \phi_{1}\left(i q^{1 / 4} e^{\xi},-i q^{1 / 4} e^{-\xi} ;-q^{1 / 2} ; q^{1 / 2}, q^{1 / 2}\right) \\
\quad-{ }_{2} \phi_{1}\left(i q^{1 / 4} e^{-\xi},-i q^{1 / 4} e^{\xi} ;-q^{1 / 2} ; q^{1 / 2}, q^{1 / 2}\right) \\
=\frac{4 i x q^{3 / 4}\left(q^{2} ; q^{2}\right)_{\infty}}{(q-1)\left(q^{1 / 2} ; q^{1 / 2}\right)_{\infty}}{ }_{2} \phi_{1}\left(q e^{2 \xi}, q e^{-2 \xi} ; q^{3} ; q^{2}, q^{2}\right),
\end{aligned}
$$

and

$$
\begin{aligned}
{ }_{2} \phi_{1}\left(i q^{1 / 4} e^{\xi},-i q^{1 / 4} e^{-\xi} ;-q^{1 / 2} ; q^{1 / 2}, q^{1 / 2}\right) \\
\quad+{ }_{2} \phi_{1}\left(i q^{1 / 4} e^{-\xi},-i q^{1 / 4} e^{\xi} ;-q^{1 / 2} ; q^{1 / 2}, q^{1 / 2}\right) \\
=\frac{2\left(q ; q^{2}\right)_{\infty}}{\left(q^{1 / 2} ; q^{1 / 2}\right)_{\infty}}{ }_{2} \phi_{1}\left(q e^{2 \xi}, q e^{-2 \xi} ; q ; q^{2}, q\right) .
\end{aligned}
$$

The above quartic transformations are new. In a private communication Mizan Rahman found direct proofs of (5.46) and (5.47) using the theory of basic hypergeometric functions.

\section{EXTREMAL MEASURES}

As we mentioned in Section 5 the solutions to the moment problem are $\{\psi(t, \sigma)\}$, where $\sigma$ is analytic in $\Im z>0$ such that $\Im \sigma(z) \leq 0$ if $\Im z>0$. Furthermore

$$
\frac{A(z)-\sigma(z) C(z)}{B(z)-\sigma(z) D(z)}=\int_{-\infty}^{\infty} \frac{d \psi(t ; \sigma)}{z-t} .
$$

where $A(x), B(x), C(x), D(x)$ are as in (5.32), (5.36), and (5.45), see also (6.11). An alternate representation of $C(x)$ is given in (6.10).

The cases $\sigma=0$ and $\sigma=-\infty$ of (6.1) are of special interest. They are

$$
\begin{gathered}
\frac{-4 x q\left(q^{2} ; q\right)_{\infty}}{\left(q e^{2 \xi}, q e^{-2 \xi} ; q^{2}\right)_{\infty}}{ }_{2} \phi_{1}\left(q e^{2 \xi}, q e^{-2 \xi} ; q^{3} ; q^{2}, q^{2}\right) \\
=\int_{-\infty}^{\infty} \frac{d \mu(t)}{x-t}, \quad x=\sinh \xi, \quad x \neq 0,
\end{gathered}
$$

and

$$
\begin{gathered}
\frac{\left(q ; q^{2}\right)_{\infty}^{2}}{x\left(q^{2} e^{2 \xi}, q^{2} e^{-2 \xi} ; q^{2}\right)_{\infty}}{ }_{2} \phi_{1}\left(q e^{2 \xi}, q e^{-2 \xi} ; q ; q^{2} q\right) \\
=\int_{-\infty}^{\infty} \frac{d \nu(t)}{x-t}, \quad x=\sinh \xi, \quad x \neq 0,
\end{gathered}
$$


where

$$
\mu(x)=\psi(x, 0), \quad \nu(x)=\psi(x, \infty) .
$$

Recall the Perron-Stieltjes inversion formula [47], [49]

$$
F(z):=\int_{-\infty}^{\infty} \frac{d \psi(t)}{z-t}, \quad \Im z \neq 0,
$$

if and only if

$$
2 \pi i[\psi(v)-\psi(u)]=\lim _{\varepsilon \rightarrow 0^{+}} \int_{u}^{v}[F(t-i \varepsilon)-F(t+i \varepsilon)] d t,
$$

and $\psi$ is normalized by

$$
\psi(u)=\frac{1}{2}\left[\psi\left(u^{+}\right)+\psi\left(u^{-}\right)\right] .
$$

Since the right sides of (6.2) and (6.3) are meromorphic functions of $x$, then both $d \mu$ and $d \nu$ are discrete measures. In this case the inversion formula (6.5) shows that the measures involved are supported on the poles of the respective right-hand sides and the masses equal the corresponding residues. Thus the support of $d \mu$ consists of all $x$ 's at which $e^{ \pm 2 \xi}=q^{2 n+1}$, that is supp $d \mu=$ $\left\{ \pm x_{n}, n=0,1, \ldots\right\}$ where

$$
x_{n}=\frac{1}{2}\left(q^{-n-1 / 2}-q^{n+1 / 2}\right), \quad n=0,1,2, \ldots .
$$

The residue of the left-hand side of (6.2) at $x=x_{n}$ is

$$
\frac{-2\left(q^{-n-1 / 2}-q^{n+1 / 2}\right) q\left(q^{2} ; q\right)_{\infty} \phi_{1}\left(q^{-2 n}, q^{2 n+2} ; q^{3} ; q^{2}, q^{2}\right)}{\left(q^{2 n+2} ; q^{2}\right)_{\infty}\left(q^{-2 n} ; q^{2}\right)_{n}\left(q^{2} ; q^{2}\right)_{\infty}} \lim _{x \rightarrow x_{n}} \frac{x-x_{n}}{1-q^{2 n+1} e^{2 \xi}} \text {. }
$$

The ${ }_{2} \phi_{1}$ can be summed by the $q$ analog of the Chu-Vandermonde sum (2.31), [25], (II.6). This shows that the aforementioned residue is

$$
\frac{\left(q^{-n-1 / 2}-q^{n+1 / 2}\right) q\left(q^{3} ; q^{2}\right)_{\infty}\left(q^{-n-1 / 2}+q^{n+1 / 2}\right)}{2\left(q^{2 n+2} ; q^{2}\right)_{\infty}\left(q^{-2 n} ; q^{2}\right)_{n}\left(q^{3} ; q^{2}\right)_{\infty}} q^{2 n(n+1)}\left(q^{1-2 n} ; q^{2}\right)_{n}
$$

which can be simplified to

$$
\frac{1}{2}\left(q ; q^{2}\right)_{\infty}\left(1+q^{2 n+1}\right) q^{2 n^{2}+n} /\left(q^{2} ; q^{2}\right)_{\infty}
$$

Similarly we calculate the residue at $-x_{n}$. Therefore

$$
\int_{-\infty}^{\infty} h_{m}(x \mid q) h_{n}(x \mid q) d \mu(x)=q^{-n(n+1) / 2}(q)_{n} \delta_{m, n},
$$

where $d \mu$ is supported at $\pm x_{n} ; n=0,1, \ldots, x_{n}$ is as in (6.6) and

$$
\mu\left( \pm x_{n}+0^{+}\right)-\mu\left( \pm x_{n}-0^{+}\right)=\frac{\left(q ; q^{2}\right)_{\infty}}{2\left(q^{2} ; q^{2}\right)_{\infty}}\left(1+q^{2 n+1}\right) q^{2 n^{2}+n}, \quad n=0,1, \ldots
$$

The inversion of (6.3) is somewhat similar. The residue at $x=0$ follows from the $q$ binomial theorem (2.28). The calculation of the remaining residues will 
be simplified if we use the following iterate of the Heine transformation, (III.3) in [25],

$$
{ }_{2} \phi_{1}(a, b ; c ; q, z)=\frac{(a b z / c ; q)_{\infty}}{(z ; q)_{\infty}}{ }_{2} \phi_{1}(c / a, c / b ; c ; q, a b z / c)
$$

to transform the ${ }_{2} \phi_{1}$ in $C(x)$. This gives

$$
C(x)={ }_{2} \phi_{1}\left(e^{-2 \xi}, e^{2 \xi} ; q ; q^{2}, q^{2}\right) .
$$

Now (6.3) takes the simplified form

$$
\frac{(q ; q)_{\infty}}{x\left(q^{2} e^{2 \xi}, q^{2} e^{-2 \xi} ; q^{2}\right)_{\infty}}{ }_{2} \phi_{1}\left(e^{-2 \xi}, e^{2 \xi} ; q ; q^{2}, q^{2}\right)=\int_{-\infty}^{\infty} \frac{d \nu(t)}{x-t} .
$$

The calculation of the residues of the left-hand side in (6.11) is now straightforward and will be omitted. The result is: $d \nu$ is a purely discrete measure supported at $0, \pm y_{n}, n=0,1, \ldots$,

$$
y_{n}=\frac{1}{2}\left(q^{-n-1}-q^{n+1}\right), \quad n=0,1,2, \ldots .
$$

The masses are given by

$$
\begin{aligned}
& \nu\left(0^{+}\right)-\nu\left(0^{-}\right)=\left(q ; q^{2}\right)_{\infty} /\left(q^{2} ; q^{2}\right)_{\infty}, \\
& \nu\left( \pm y_{n}+0^{+}\right)-\nu\left( \pm y_{n}-0^{+}\right)=\frac{\left(q ; q^{2}\right)_{\infty}}{2\left(q^{2} ; q^{2}\right)_{\infty}}\left(1+q^{2 n+2}\right) q^{2 n^{2}+3 n+1} \text {, } \\
& n=0,1,2, \ldots .
\end{aligned}
$$

It is of interest to verify directly that $d \mu$ and $d \nu$ are probability measures. It is also interesting to see what (6.2) and (6.3) are really saying. The total mass of $d \mu$ is

$$
\frac{\left(q ; q^{2}\right)_{\infty}}{\left(q^{2} ; q^{2}\right)_{\infty}} \sum_{n=0}^{\infty}\left(1+q^{2 n+1}\right) q^{2 n^{2}+n}
$$

that is

$$
\frac{\left(q ; q^{2}\right)_{\infty}}{\left(q^{2} ; q^{2}\right)_{\infty}}\left[\sum_{n=0}^{\infty} q^{2 n^{2}+n}+\sum_{n=1}^{\infty} q^{(2 n-1) n}\right]=\frac{\left(q ; q^{2}\right)_{\infty}}{\left(q^{2} ; q^{2}\right)_{\infty}} \sum_{-\infty}^{\infty} q^{2 n^{2}+n}
$$

This can be summed from the Jacobi triple product identity, (II.28) in [25], namely

$$
\sum_{-\infty}^{\infty} q^{k^{2}} z^{k}=\left(q^{2},-q z,-q / z ; q^{2}\right)_{\infty}
$$

Thus the total $\mu$ mass is

$$
\frac{\left(q ; q^{2}\right)_{\infty}}{\left(q^{2} ; q^{2}\right)_{\infty}}\left(q^{4},-q^{2},-q ; q^{4}\right)=\frac{\left(q ; q^{2}\right)_{\infty}}{\left(q^{2} ; q^{2}\right)_{\infty}}\left(q^{4} ; q^{4}\right)_{\infty}\left(-q ; q^{2}\right)_{\infty}=1
$$


The total $\nu$ mass is

$$
\begin{aligned}
& \frac{\left(q ; q^{2}\right)_{\infty}}{\left(q^{2} ; q^{2}\right)_{\infty}}\left[1+\sum_{0}^{\infty}\left(1+q^{2 n+2}\right) q^{2 n^{2}+3 n+1}\right] \\
& \quad=\frac{\left(q ; q^{2}\right)_{\infty}}{\left(q^{2} ; q^{2}\right)_{\infty}}\left[1+\sum_{1}^{\infty}\left(1+q^{2 n}\right) q^{n(2 n-1)}\right] \\
& \quad=\frac{\left(q ; q^{2}\right)_{\infty}}{\left(q^{2} ; q^{2}\right)_{\infty}} \sum_{-\infty}^{\infty} q^{2 n^{2}-n}=\frac{\left(q ; q^{2}\right)_{\infty}}{\left(q^{2} ; q^{2}\right)_{\infty}} \sum_{-\infty}^{\infty} q^{2 n^{2}+n}=1,
\end{aligned}
$$

as before. This verifies that both $d \mu$ and $d \nu$ are probability measures.

It is known from the theory of moment problem that the entire functions $A$, $B, C, D$ satisfy the Wronskian identity

$$
A(x) D(x)-B(x) C(x)=1 \text {. }
$$

In the present case this identity is equivalent to

$$
\begin{aligned}
& \frac{4 x^{2} q\left(q^{2} e^{2 \xi}, q^{2} e^{-2 \xi} ; q^{2}\right)_{\infty}}{(1-q)\left(q ; q^{2}\right)_{\infty}^{2}}{ }_{2} \phi_{1}\left(q e^{2 \xi}, q e^{-2 \xi} ; q^{3} ; q^{2}, q^{2}\right) \\
& +\frac{\left(q e^{2 \xi}, q e^{-2 \xi} ; q^{2}\right)_{\infty}}{\left(q, q ; q^{2}\right)_{\infty}}{ }_{2} \phi_{1}\left(e^{2 \xi}, e^{-2 \xi} ; q ; q^{2}, q^{2}\right)=1 .
\end{aligned}
$$

This follows from the nonterminating form of the $q$-Chu-Vandermonde sum, formula (II.23) in [25],

$$
\begin{aligned}
{ }_{2} \phi_{1}(a, b ; c ; q, q) & +\frac{(q / c, a, b ; q)_{\infty}}{(c / q, a q / c, b q / c ; q)_{\infty}}{ }_{2} \phi_{1}\left(a q / c, b q / c ; q^{2} / c ; q, q\right) \\
& =\frac{(q / c, a b q / c ; q)_{\infty}}{(a q / c, b q / c ; q)_{\infty}} .
\end{aligned}
$$

We now discuss qualitative properties of the extremal measures, so we consider $\sigma \in[-\infty, \infty]$. It is clear from (5.4) that in general, the extremal measures are discrete and are supported at the zeros of $B(x)-\sigma D(x)$. These zeros are all real and simple. It is interesting to note that the $h_{n}$ 's are symmetric, that is $h_{n}(-x)=(-1)^{n} h_{n}(x)$, but the masses of the extremal measures are symmetric about the origin only when $\sigma=0, \pm \infty$. This is so because the Stieltjes transform $\int_{-\infty}^{\infty} \frac{d \psi(t)}{x-t}$ of a normalized symmetric measure $(d \psi(-t)=d \psi(t))$ is always an odd function of $x$ but it is clear that $A(x)$ and $D(x)$ are odd functions but $B(x)$ and $C(x)$ are even functions.

Let $\left\{x_{n}(\sigma)\right\}_{-\infty}^{\infty}$ be the zeros of $B(x)-\sigma D(x)$ arranged in increasing order

$$
\cdots<x_{-n}(\sigma)<\sigma_{-n+1}(\sigma)<\cdots<x_{n}(\sigma)<x_{n+1}(\sigma)<\cdots .
$$

The zeros of $D(x)$ are $\left\{x_{n}(-\infty)\right\}_{-\infty}^{\infty}$ and are labeled as

$$
\cdots<x_{-2}(-\infty)<x_{-1}(-\infty)<x_{0}(-\infty)=0<x_{1}(-\infty)<\cdots .
$$

In general it is known that $x_{n}(\sigma)$ is a real analytic strictly increasing function of $\sigma$ and increases from $x_{n}(-\infty)$ to $x_{n+1}(-\infty)$ as $\sigma$ increases from $-\infty$ to $+\infty$. Furthermore the sequences $\left\{x_{n}\left(\sigma_{1}\right)\right\}$ and $\left\{x_{n}\left(\sigma_{2}\right)\right\}$ interlace when 
$\sigma_{1} \neq \sigma_{2}$. This is part of Theorem 2.13, page 60 in [47]. A proof is in [49], see Theorem 10.41, pp 584-589. In the case we are interested in (6.12) and (6.6) give

$$
\begin{gathered}
x_{n}(-\infty)=\frac{1}{2}\left(q^{-n}-q^{n}\right), \quad x_{n}(\infty)=x_{n+1}(-\infty), \\
x_{n}(0)=\frac{1}{2}\left(q^{-n-1 / 2}-q^{n+1 / 2}\right), \quad n \geq 0, \\
x_{-n}(0)=x_{n-1}(0), \quad n>0 .
\end{gathered}
$$

We know that $B(z) / D(z)$ is a meromorphic function with only real zeros and poles. We shall prove later that $B(z) / D(z)$ is increasing on any open interval whose end points are consecutive poles of $B(z) / D(z)$. For $\sigma \in(-\infty, \infty)$ define $\eta=\eta(\sigma)$ as the unique solution of

$$
\sigma=B(\sinh \eta) / D(\sinh \eta), \quad 0=x_{0}(-\infty)<\sinh \eta<x_{1}(-\infty) .
$$

We define $\eta( \pm \infty)$ by

$$
\eta(-\infty)=0, \quad \eta(\infty)=x_{1}(-\infty)=x_{0}(\infty)=\left(q^{-1}-q\right) / 2 .
$$

The next step is to invert $(6.1)$ and find $\psi(t, \sigma)$ explicitly. The approach followed here uses product formulas for theta functions. An alternate derivation will be given in Section 9. We follow the notation in Whittaker and Watson [52], Section 21. Recall that the four theta functions have the infinite product representations [52], Section $\S 21.3$,

$$
\begin{aligned}
& \vartheta_{1}(z)=2 q^{1 / 4} \sin z\left(q^{2}, q^{2} e^{2 i z}, q^{2} e^{-2 i z} ; q^{2}\right)_{\infty}, \\
& \vartheta_{3}(z)=\left(q^{2},-q e^{2 i z},-q e^{-2 i z} ; q^{2}\right)_{\infty}, \\
& \vartheta_{2}(z)=2 q^{1 / 4} \cos z\left(q^{2},-q^{2} e^{2 i z},-q^{2} e^{-2 i z} ; q^{2}\right)_{\infty}, \\
& \vartheta_{4}(z)=\left(q^{2}, q e^{2 i z}, q e^{-2 i z} ; q^{2}\right)_{\infty} .
\end{aligned}
$$

It is clear that $B(\sinh \xi)$ and $D(\sinh \xi)$ are theta functions. Indeed

$$
\begin{gathered}
D(\sinh \xi)=\vartheta_{1}(i \xi) /\left[2 i q^{1 / 4}(q ; q)_{\infty}\left(q^{2} ; q^{2}\right)_{\infty}\right], \\
B(\sinh \xi)=-\vartheta_{4}(i \xi) /\left[(q ; q)_{\infty}\left(q ; q^{2}\right)_{\infty} .\right.
\end{gathered}
$$

With the choice of $\sigma$ made in (6.19) the Stieltjes transform of $d \psi(t, \sigma)$ becomes

$$
\frac{A(\sinh \xi)-\sigma C(\sinh \xi)}{B(\sinh \xi)-\sigma D(\sinh \xi)}=\frac{A(\sinh \xi) D(\sinh \eta)-B(\sinh \eta) C(\sinh \xi)}{B(\sinh \xi) D(\sinh \eta)-B(\sinh \eta) D(\sinh \xi)}
$$


Theorem 6.1. The cross product $B(\sinh \xi) D(\sinh \eta)-B(\sinh \eta) D(\sinh \xi)$ has the infinite product representation

$B(\sinh \xi) D(\sinh \eta)-B(\sinh \eta) D(\sinh \xi)$

$$
=\frac{-1}{2 a(q ; q)_{\infty}} \prod_{n=0}^{\infty}\left(1-2 a q^{n} \sinh \xi-a^{2} q^{2 n}\right)\left(1+2 a^{-1} q^{n+1} \sinh \xi-q^{2 n+2} a^{-2}\right),
$$

where $\eta$ and $\sigma$ are related by (6.19) and

$$
a=e^{-\eta} \text {. }
$$

Proof. The relationships (6.22) and (6.23) show that the above cross product is

$$
\left[2 i q^{1 / 4}(q ; q)_{\infty}^{2}\left(q, q^{2} ; q^{2}\right)_{\infty}\right]^{-1}\left[\vartheta_{1}(i \xi) \vartheta_{4}(i \eta)-\vartheta_{1}(i \eta) \vartheta_{4}(i \xi)\right]
$$

The product formula, [52], p. 488

$\vartheta_{1}(y \pm z) \vartheta_{4}(y \mp z) \vartheta_{2}(0) \vartheta_{3}(0)=\vartheta_{1}(y) \vartheta_{4}(y) \vartheta_{2}(z) \vartheta_{3}(z) \pm \vartheta_{2}(y) \vartheta_{3}(y) \vartheta_{1}(z) \vartheta_{4}(z)$, enables us to reduce the left-hand side in (6.25) to

$$
\frac{\vartheta_{2}(i(\xi+\eta) / 2) \vartheta_{3}(i(\xi+\eta) / 2) \vartheta_{1}(i(\xi-\eta) / 2) \vartheta_{4}(i(\xi-\eta) / 2)}{2 i \sqrt{q}(q ; q)_{\infty}^{3}\left(q^{2},-q,-q^{2} ; q^{2}\right)_{\infty}^{2}} .
$$

The infinite product representations (6.20) and (6.21) simplify the latter expression to

$2 \sinh ((\xi-\eta) / 2) \cosh ((\xi+\eta) / 2)\left(-q e^{\xi+\eta},-q e^{-\xi-\eta}, q e^{\xi-\eta}, q e^{\eta-\xi} ; q\right)_{\infty} /(q ; q)_{\infty}$,

which simplifies further and we obtain (6.25).

Therefore the zeros of the left side of $(6.25)$ are

$$
x_{n}(\sigma)=\frac{1}{2}\left(q^{-n} a^{-1}-a q^{n}\right), \quad n=0, \pm 1, \ldots,
$$

From, (6.19) it is clear that $0<\eta<-\ln q$, hence

$$
q<a<1 \text {. }
$$

In fact there is no loss of generality in assuming $q \leq a<1$ in (6.27) since the set of zeros is invariant under replacing $a$ by $a q^{j}$, for any integer $j$. An extremal measure $d \psi(x, \sigma)$ will be supported at $\left\{x_{n}(\sigma) \mid-\infty<n<\infty\right\}$. To find the mass at $x_{n}(\sigma)$ we either compute the residue of either side of (6.24) or apply Theorem 2.13, page 60 in [47]. The aforementioned theorem asserts that the mass concentrated at $x_{n}(\sigma)$ is $1 / \sum_{k=0}^{\infty} p_{k}^{2}\left(x_{n}(\sigma)\right)$, where $\left\{p_{k}(x)\right\}$ are the orthonormal polynomials. Set

$$
m_{n}=\text { mass concentrated at } x_{n}(\sigma) .
$$

In our case, the orthonormal polynomials are $\left\{q^{n(n+1) / 4} h_{n}(x \mid q) / \sqrt{(q ; q)_{n}}\right\}$. Thus

$$
m_{n}=1 / \sum_{k=0}^{\infty} q^{k(k+1) / 2} h_{k}^{2}\left(x_{n}(\sigma)\right) /(q ; q)_{k} .
$$


The series on the right-hand side is a special case of the Poisson kernel (2.8). Therefore

$$
m_{n}=1 /\left(-q^{1-2 n} a^{-2},-a^{2} q^{2 n+1}, q ; q\right)_{\infty} .
$$

After routine manipulations we obtain

$$
\left\{\begin{array}{l}
m_{n}=a^{4 n} q^{n(2 n-1)}\left(1+a^{2} q^{2 n}\right) /\left(-a^{2},-q / a^{2}, q ; q\right)_{\infty}, \quad n \geq 0, \\
m_{-n}=a^{-4 n+2} q^{n(2 n-1)}\left(1+q^{2 n} / a^{2}\right) /\left(-a^{2},-q / a^{2}, q ; q\right)_{\infty}, \quad n \geq 0 .
\end{array}\right.
$$

Both cases can be consolidated in single form, namely

$$
m_{n}=a^{4 n} q^{n(2 n-1)}\left(1+a^{2} q^{2 n}\right) /\left(-a^{2},-q / a^{2}, q ; q\right)_{\infty}, \quad n=0, \pm 1, \pm 2, \ldots
$$

This establishes the orthogonality relation

$$
\sum_{n=-\infty}^{\infty} m_{n} h_{r}\left(x_{n}(\sigma) \mid q\right) h_{s}\left(x_{n}(\sigma) \mid q\right)=q^{-r(r+1) / 2} \delta_{r, s} /(q ; q)_{r}
$$

the masses $m_{n}$ are given by (6.30), the mass points $\left\{x_{n}(\sigma)\right\}$ are as in (6.27).

Recall that the measures are normalized to have a total mass equal to unity. Therefore

$$
\begin{aligned}
& \left(-q a^{2},-q a^{-2}, q ; q\right)_{\infty} \\
& =\sum_{n=0}^{\infty} a^{4 n} q^{n(2 n-1)} \frac{\left(1+a^{2} q^{2 n}\right)}{1+a^{2}}+\sum_{n=1}^{\infty} a^{-4 n} q^{n(2 n-1)} \frac{\left(a^{2}+q^{2 n}\right)}{1+a^{2}} \\
& =\frac{1}{1+a^{2}}\left[\sum_{0}^{\infty} a^{4 n} q^{n(2 n-1)}+\sum_{1}^{\infty} a^{4 n+2} q^{n(2 n+1)}\right. \\
& \left.\quad+\sum_{-1}^{-\infty} a^{4 n+2} q^{n(2 n+1)}+\sum_{-1}^{-\infty} a^{4 n} q^{n(2 n-1)}\right] \\
& =\frac{1}{1+a^{2}}\left[\sum_{-\infty}^{\infty} a^{4 n} q^{n(2 n-1)}+\sum_{-\infty}^{\infty} a^{4 n+2} q^{n(2 n+1)}\right] .
\end{aligned}
$$

Observe that the first and second sums above are the even and odd parts of $\sum_{-\infty}^{\infty}\left(a^{2} / \sqrt{q}\right)^{n} q^{n^{2} / 2}$, respectively. Therefore $\int_{-\infty}^{\infty} d \psi(x, \sigma)=1$ is equivalent to the summation theorem

$$
\sum_{-\infty}^{\infty} z^{n} p^{n^{2}}=\left(p^{2},-p z,-p / z ; p^{2}\right)_{\infty}
$$

Clearly (6.32) is the Jacobi triple product identity (6.14). It is important to note that we have not used (6.32) in any computations leading to (6.32) and as such we obtain the Jacobi triple product identity as a by-product of our analysis. 
We now give an alternate derivation of the masses in (6.30). This alternate calculation will be used in Section 7 to analyze other spectral measures for the $h_{n}$ 's. From (5.32), (5.36), (5.45), and (6.10) we get

$$
\begin{aligned}
& A(\sinh \xi) D(\sinh \eta)-B(\sinh \eta) C(\sinh \xi) \\
& \quad=\frac{4 q \sinh \xi \sinh \eta\left(q^{2} ; q^{2}\right)_{\infty}}{(1-q)\left(q ; q^{2}\right)_{\infty}(q ; q)_{\infty}}\left(q^{2} e^{2 \eta}, q^{2} e^{-2 \eta} ; q^{2}\right)_{\infty} \\
& \quad \times{ }_{2} \phi_{1}\left(q e^{2 \xi}, q e^{-2 \xi} ; q^{3} ; q^{2}, q^{2}\right) \\
& \quad+\left(q e^{2 \eta}, q e^{-2 \eta} ; q^{2}\right)_{\infty}\left(q ; q^{2}\right)_{\infty}^{-2}{ }_{2} \phi_{1}\left(e^{2 \xi}, e^{-2 \xi} ; q ; q^{2}, q^{2}\right) .
\end{aligned}
$$

When $x=x_{n}(\sigma), \sigma$ becomes $\sigma_{n}$ where $e^{\xi_{n}}=q^{-n} / a$, with $a$ as in (6.26).

Theorem 6.2. When $e^{\xi_{n}}=q^{-n} / a$ then

$$
A(\sinh \xi) D(\sinh \eta)-B(\sinh \eta) C(\sinh \xi)=(-1)^{n} a^{2 n} q^{n^{2}} .
$$

Proof. Apply (6.33) to see that the left-hand side of (6.34) is

$$
\begin{aligned}
& \frac{\left(a^{2} q^{n}-q^{-n}\right) q\left(a^{-2}, q^{2} a^{2} ; q^{2}\right)_{\infty}}{(1-q)\left(q ; q^{2}\right)_{\infty}^{2}}{ }_{2} \phi_{1}\left(q^{1-2 n} / a^{2}, q^{1+2 n} a^{2} ; q^{3} ; q^{2}, q^{2}\right) \\
& +\frac{\left(q a^{2}, q / a^{2} ; q^{2}\right)_{\infty}}{\left(q ; q^{2}\right)_{\infty}^{2}}{ }_{2} \phi_{1}\left(q^{-2 n} / a^{2}, q^{2 n} a^{2} ; q ; q^{2}, q^{2}\right),
\end{aligned}
$$

which reduces to

$$
\begin{aligned}
& \frac{q\left(a^{2} q^{n}-q^{-n}\right)\left(a^{2} q^{2}, a^{-2} ; q^{2}\right)_{\infty}}{(1-q)\left(q ; q^{2}\right)_{\infty}^{2}}{ }_{2} \phi_{1}\left(q^{1-2 n} / a^{2}, q^{1+2 n} a^{2} ; q^{3} ; q^{2}, q^{2}\right) \\
& \quad+\frac{\left(q a^{2}, q / a^{2} ; q^{2}\right)_{\infty}}{\left(a^{2} q^{2 n+1}, q^{1-2 n} / a^{2} ; q^{2}\right)_{\infty}} \\
& \quad-\frac{\left(q a^{2}, q / a^{2}, q, q^{-2 n} / a^{2}, a^{2} q^{2 n} ; q^{2}\right)_{\infty}}{\left(q, q, 1 / q, q^{1-2 n} / a^{2}, a^{2} q^{2 n+1} ; q^{2}\right)_{\infty}} \\
& \quad \times{ }_{2} \phi_{1}\left(q^{1-2 n} / a^{2}, q^{1+2 n} a^{2} ; q^{3} ; q^{2}, q^{2}\right)
\end{aligned}
$$

upon the application of the nonterminating $q$ analog of the Chu-Vandermonde sum (6.15). After straightforward simplification we see that the ${ }_{2} \phi_{1}$ 's cancel and we obtain (6.34).

The extremal measures arise when $\sigma(z)$ is a real constant. Since $\overline{\sigma(z)}=$ $\sigma(\bar{z})$, we cannot take $\sigma(z)$ to be identically equal to a nonreal constant. This suggests that the next step is to consider

$$
\sigma(z)=\alpha e^{-i \beta}, \Im z>0, \quad \sigma(z)=\alpha e^{i \beta}, \Im z<0, \text { for } \alpha>0,0 \leq \beta \leq \pi .
$$

The extremal measures correspond to the cases $\beta=0, \pi$. The spectral measures associated with the function $\sigma$ of $(6.35)$ will be found explicitly in the next section. 


\section{Applications}

An orthogonality relation of a sequence of orthogonal polynomials or functions is usually equivalent to the explicit evaluation of a series or a definite integral. There is a hierarchy of orthogonal polynomials indicated in Askey's Tableau [34], and most books on special functions include a similar list of summation formulas and explicit evaluations of integrals. The intertwining of orthogonality relations and explicit sums and integrals is well illustrated in the excellent book [25]. The discovery of a new orthogonality relation may lead to new summation theorems [25], Section 7, or shed new light on known identities. The analysis we used in Section 6 led naturally to the Jacobi triple product identity, being $\int_{-\infty}^{\infty} d \psi(t, \sigma)=1$. In retrospect, this may not be very surprising because the orthogonality of $\left\{H_{n}(x \mid q)\right\}$ follows from the Jacobi triple product identity, as was realized in [3] and [7]. This indicates that the $q$-Hermite polynomials for $0<q<1$, or $q>1$ exist at the level of the Jacobi triple product identity. A $q$ analog of the Askey Tableau is in preparation. It has the $q$-Hermite polynomials at the bottom of the chart with the $q$-Racah and AskeyWilson polynomials at the top. Ismail and Stanton [29] realized the power of Rogers's linearization formula (1.13) by combining (1.13) with the orthogonality of $\left\{H_{n}(x \mid q)\right\}$ to evaluate the Askey-Wilson integral which is the key step in establishing the orthogonality of the Askey-Wilson polynomials. Thus the linearization formula of Rogers (1.13) provides a magic lift from the bottom level of a $q$-Askey's tableau to its top level.

Convinced of the power of (1.13), we embarked upon the task of identifying the summation theorems behind the evaluation of the integral (1.18) and (6.1). We already saw in Section 3 that the evaluation of $I\left(t_{1}, t_{2}, t_{3}, t_{4}\right)$ for general extremal measures is equivalent to Bailey's sum of a very well-poised ${ }_{6} \psi_{6}$, stated in (3.17). The full ${ }_{6} \psi_{6}$ sum is an interesting sum near the top of the list of explicit sums.

In Section 5 of [10], Richard Askey pointed out that Rogers [45, page 30], actually found the sum of a very well-poised ${ }_{6} \phi_{5}$. This is the special case $b=a$ of Bailey's ${ }_{6} \psi_{6}$ sum. Askey and Ismail [13] used the identity theorem for analytic functions to prove Bailey's ${ }_{6} \psi_{6}$ from the ${ }_{6} \phi_{5}$ summation theorem. Thus, the solution of the Hamburger moment problem associated with $\left\{h_{n}(x \mid q)\right\}$ is very rich, and when we combined the classical theory of indeterminate Hamburger moment problems with the linearization formula of Rogers we were led to the ${ }_{6} \psi_{6}$ in a natural and straightforward way.

Next we consider (6.1) with $\sigma$ in the extended real number system. The use of (6.24), (6.25), (6.10), and (5.45) imply

$$
\begin{aligned}
& \frac{2 q \sinh \xi}{a(1-q)}\left(a^{2}, q^{2} / a^{2} ; q^{2}\right)_{\infty 2} \phi_{1}\left(q e^{2 \xi}, q e^{-2 \xi} ; q^{3} ; q^{2}, q^{2}\right) \\
& \quad+\left(q a^{2}, q / a^{2} ; q^{2}\right)_{\infty 2} \phi_{1}\left(e^{2 \xi}, e^{-2 \xi} ; q ; q^{2}, q^{2}\right) \\
& =-\frac{\left(1-a e^{\xi}\right)\left(1+a e^{-\xi}\right)\left(a q e^{\xi},-a q e^{-\xi},-q e^{\xi} / a, q e^{-\xi} / a ; q^{2}\right)_{\infty}}{2 a\left(1+a^{2}\right)\left(-q a^{2},-q / a^{2}, q ; q\right)_{\infty}} \\
& \quad \times \sum_{-\infty}^{\infty} \frac{a^{4 n} q^{2 n^{2}-n}\left(1+a^{2} q^{2 n}\right)}{x+\left(a q^{n}-q^{-n} / a\right) / 2} .
\end{aligned}
$$


After some manipulations we obtain the quadratic transformation formula

$$
\left\{\begin{array}{c}
\frac{q\left(e^{\xi}-e^{-\xi}\right)}{a(1-q)}\left(a^{2}, q^{2} / a^{2} ; q^{2}\right)_{\infty 2} \phi_{1}\left(q e^{2 \xi}, q e^{-2 \xi} ; q^{3} ; q^{2}, q^{2}\right) \\
\quad+\left(q a^{2}, q / a^{2} ; q^{2}\right)_{\infty 2} \phi_{1}\left(e^{2 \xi}, e^{-2 \xi} ; q ; q^{2}, q^{2}\right) \\
=\frac{\left(a q e^{\xi},-a q e^{-\xi},-q e^{\xi} / a, q e^{-\xi} / a ; q^{2}\right)_{\infty}}{\left(-q a^{2},-q / a^{2}, q ; q\right)_{\infty}} \\
\quad \times \sum_{-\infty}^{\infty} \frac{\left(i a q,-i a q, a e^{\xi},-a e^{-\xi} ; q\right)_{n}}{\left(-i a, i a,-q a e^{-\xi}, q a e^{\xi} ; q\right)_{n}} a^{4 n} q^{2 n^{2}}
\end{array}\right.
$$

So far we have only discussed the extremal measures and they all are purely discrete. We now discuss other measures which are not extremal. Askey [12] found an absolutely continuous measure with respect to which the $h_{n}$ 's are orthogonal. He proved

$$
\int_{-\infty}^{\infty} \frac{h_{m}(\sinh \xi \mid q) h_{n}(\sinh \xi \mid q)}{(-\ln q)\left(q,-q e^{2 \xi},-q e^{-2 \xi}\right)_{\infty}} d \xi=(q ; q)_{n} q^{-n(n+1) / 2} \delta_{m, n}
$$

With $d \psi(x)=\left[(-\ln q)\left(q,-q e^{2 \xi},-q e^{-2 \xi}\right)_{\infty}\right]^{-1} d \xi$ in (1.18) we immediately get

$$
\int_{-\infty}^{\infty} \frac{\prod_{j=1}^{4}\left(-t_{j} e^{\xi}, t_{j} e^{-\xi}\right)_{\infty} d \xi}{(-\ln q)\left(q,-q e^{2 \xi},-q e^{-2 \xi}\right)_{\infty}}=\left[\prod_{1 \leq j<k \leq 4}\left(-t_{j} t_{k} / q\right)_{\infty}\right] /\left(t_{1} t_{2} t_{3} t_{4} q^{-3}\right)_{\infty}
$$

Askey gave a proof of (7.3) in [10]. Although the Stieltjes transform of Askey's measures must have the form (6.1) we do not know the corresponding function $\sigma$ explicitly. It is interesting that (7.3) follows from (7.2) for free from our evaluation (3.8). It is worth noting that Askey's measure is a discrete analogue of the exremal measures. To see this use (2.8) and the fact

$$
m_{n}=1 / \sum_{k=0}^{\infty} q^{k(k+1) / 2} h_{k}^{2}\left(x_{n}(\sigma)\right) /(q ; q)_{k}
$$

to see that $m_{n}$ is a constant multiple of $1 /\left(-q e^{2 \xi_{n}},-q e^{-2 \xi_{n}}\right)_{\infty}$, where $x_{n}(\sigma)=$ $\sinh \xi_{n}$. We also note that one can prove formula (7.3) directly by making a change of variable $v=e^{-\xi}$, write the resulting integral as $\sum_{-\infty}^{\infty} \int_{q^{j+1}}^{q^{j}}$, replace $v$ by $w q^{j}$, interchange integration (which is now on $[q, 1]$ ) and summation, then evaluate the sum using the ${ }_{6} \psi_{6}$ sum (3.17) [27]. It is not surprising that the ${ }_{6} \psi_{6}$ sum is what is behind (7.3), since after all, the evaluation of the ${ }_{6} \psi_{6}$ sum is equivalent to (3.8) when $d \psi$ in (1.18) is any extremal measure.

In Section 6 we found it useful to parameterize the extremal measures using a parameter $\eta$, instead of the parameter $\sigma$, where $\sigma=B(\sinh \eta) / D(\sinh \eta)$. We will utilize this idea and obtain one additional measure for the $q^{-1}$ Hermite polynomials.

Theorem 7.1. Let

$$
\begin{aligned}
f(z) & :=-\left(q ; q^{2}\right)_{\infty} B(z) /\left[\left(q^{2} ; q^{2}\right)_{\infty} D(z)\right] \\
& =z^{-1}\left(q e^{2 \xi}, q e^{-2 \xi} ; q^{2}\right)_{\infty} /\left(q^{2} e^{2 \xi}, q^{2} e^{-2 \xi} ; q^{2}\right)_{\infty}, \quad z=\sinh \xi .
\end{aligned}
$$


Then the function $f(z)$ is a meromorphic function that maps the open upper half plane to the open lower half plane. Furthermore $f(\sqrt{z})$ is analytic in the open upper half plane and maps it to the open lower half plane.

Proof. It is clear from (5.32) and (5.36) that $f(z)$ is meromorphic and its only essential singularity is at $z=\infty$. We derive a Mittag-Leffler expansion by integrating $f(z) /(z-x)$ over a suitable contour, [52], Section 7.4. Littlewood's asymptotic formulas, [35] show that the boundedness conditions sufficient for the application of this method are satisfied. The poles of $f$ are at the points $x_{n}=\left(q^{-n}-q^{n}\right) / 2, n=0, \pm 1, \ldots$, so the corresponding values of $e^{\xi}$ are $q^{\mp n}$. Since $f$ is an odd function the residues at $\pm\left(q^{-n}-q^{n}\right) / 2$ are equal. The residue at $x_{n}=\left(q^{-n}-q^{n}\right) / 2$ is

$$
\begin{aligned}
& -\frac{\left(q^{n}+q^{-n}\right)\left(q^{1-2 n}, q^{1+2 n} ; q^{2}\right)_{\infty} /\left(q^{-n}-q^{n}\right)}{2\left(q^{2-2 n} ; q^{2}\right)_{n-1}\left(q^{2}, q^{2 n+2} ; q^{2}\right)_{\infty}} \\
& \quad=\frac{\left(q^{1-2 n}, q^{2}\right)_{n}\left(q ; q^{1+2 n} ; q^{2}\right)_{\infty}}{\left(q^{-2 n} ; q^{2}\right)_{n}\left(q^{2}, q^{2 n+2} ; q^{2}\right)_{\infty}} \frac{\left.q^{-n}+q^{n}\right)}{2}=\frac{\left(q ; q^{2}\right)_{\infty}^{2}}{\left(q^{2} ; q^{2}\right)_{\infty}^{2}} \frac{q^{-n}+q^{n}}{2} .
\end{aligned}
$$

The residue at $x=0$ is the case $n=0$. Therefore we have the Mittag-Leffler expansion

$$
\begin{aligned}
f(z)=\frac{\left(q ; q^{2}\right)_{\infty}^{2}}{\left(q^{2} ; q^{2}\right)_{\infty}^{2}} & \left\{\frac{1}{z}+\sum_{n=1}^{\infty} \frac{q^{n}+q^{-n}}{2}\right. \\
& \left.\times\left[\frac{1}{z-\left(q^{-n}-q^{n}\right) / 2}+\frac{1}{z+\left(q^{-n}-q^{n}\right) / 2}\right]\right\} .
\end{aligned}
$$

Now (7.5) implies $\Im f(z)$ is a positive multiple of $\Im \bar{z}$ when $z$ is the open upper (lower) half planes. This completes the proof.

Note that (7.5) simplifies to

$$
f(z)=\frac{\left(q ; q^{2}\right)_{\infty}^{2}}{\left(q^{2} ; q^{2}\right)_{\infty}^{2}}\left[\frac{1}{z}+\sum_{n=1}^{\infty} \frac{\left(q^{n}+q^{-n}\right) z}{z^{2}-\left(q^{-n}-q^{n}\right)^{2} / 4}\right] .
$$

Another Proof of Theorem 7.1. We prove (7.6) directly by writing the right-hand side as

$$
\begin{aligned}
& \frac{z\left(q ; q^{2}\right)_{\infty}^{2}}{\left(q^{2} ; q^{2}\right)_{\infty}^{2}} \sum_{-\infty}^{\infty} \frac{\left(q^{n}+q^{-n}\right) / 2}{z^{2}-\left(q^{-n}-q^{n}\right)^{2} / 4}=\frac{-2 z\left(q ; q^{2}\right)_{\infty}^{2}}{\left(q^{2} ; q^{2}\right)_{\infty}^{2}} \sum_{-\infty}^{\infty} \frac{q^{n}\left(1+q^{2 n}\right)}{1-2\left(2 z^{2}+1\right) q^{2 n}+q^{4 n}} \\
& =\frac{-4 z\left(q ; q^{2}\right)_{\infty}^{2}}{\left(q^{2} ; q^{2}\right)_{\infty}^{2}\left(1-e^{2 \xi}\right)\left(1-e^{-2 \xi}\right)}{ }^{2} \psi_{6}\left(\begin{array}{c}
i q,-i q, e^{\xi},-e^{\xi}, e^{-\xi},-e^{-\xi} \\
i,-i,-q e^{-\xi}, q e^{-\xi},-q e^{\xi}, q e^{\xi}
\end{array} \mid q, q\right) .
\end{aligned}
$$

Using the ${ }_{6} \psi_{6}$ sum $(3.17)$ and the fact $(-q ; q)_{\infty}\left(q ; q^{2}\right)_{\infty}=1$ we simplify the last expression to become $f(z)$ and the proof of (7.6) is complete.

Theorem 7.2. Let $g(z)$ be an analytic function in the open upper half plane and maps that region into itself. Then, for any indeterminate moment problem, the 
functions

$$
h(z):=-B(g(z)) / D(g(z)), \quad w(z):=-C(g(z)) / A(g(z))
$$

are analytic in $\Im z>0$ and map it into the closed lower half plane.

Proof. Let $\psi(t ; 0)$ and $\psi(t ;-\infty)$ be the extremal measures corresponding to $\sigma=0$ and $\sigma=-\infty$; respectively. Clearly $\frac{1}{2}[\psi(t ; 0)+\psi(t ;-\infty)]$ is a solution of the same moment problem so its Stieltjes transform must be $[A(z)-\sigma(z) C(z)] /[B(z)-\sigma(z) D(z)]$, for a suitable function $\sigma(z)$. Thus

$$
\begin{aligned}
\frac{A(z)-\sigma(z) C(z)}{B(z)-\sigma(z) D(z)} & =\frac{1}{2} \int_{-\infty}^{\infty} \frac{d \psi(t ; 0)}{z-t}+\frac{1}{2} \int_{-\infty}^{\infty} \frac{d \psi(t ;-\infty)}{z-t} \\
& =\frac{1}{2}\left[\frac{A(z)}{B(z)}+\frac{C(z)}{D(z)}\right] .
\end{aligned}
$$

This gives $\sigma(z)=-B(z) / D(z)$, and this $\sigma$ must satisfy $\Im \sigma(z) \leq 0$ if $0 \leq$ $\Im z$, see [47], Theorem 2.12, page 57. This proves the first part of our assertion. To prove the second part, first observe that the computation of $B(z)$ and $D(z)$ dealt with solutions to (5.1) satisfying the initial conditions (5.2). It is easy to see that the moment problem associated with

$$
w_{n+1}(z)=\left(z-\alpha_{n+2}\right) w_{n}(z)-\beta_{n+1} w_{n-1}(z)
$$

is indeterminate if the moment problem associated with $(5.1)$ is indeterminate. Furthermore the functions $B(z)$ and $D(z)$ for the moment problem associated with $\left\{w_{n}(z)\right\}$ are $C(z)$ and $A(z)$, respectively, of the moment problem arising from (5.1). This follows from (5.5)-(5.8). Thus the second part of the theorem follows from the first part.

It is clear that Theorem 7.2 can be useful in constructing explicit measures for indeterminate moment problems from the knowledge of $A(z), B(z), C(z)$, $D(z)$. The next Theorem will be useful in finding the absolutely continuous component of $d \psi$, for a general indeterminate moment problem, from the Stieltjes transform of $d \psi$ as given in (6.1).

Theorem 7.3. Let $\sigma$ in (6.1) be analytic in $\Im z>0$ and map it into $\Im \sigma(z) \leq 0$. In addition, assume $\overline{\sigma(z)}=\sigma(\bar{z})$. If $\psi(x, \sigma)$ does not have a jump at $x$ and $\sigma(x \pm i 0)$ exist then

$$
\frac{d \psi(x ; \sigma)}{d x}=\frac{\sigma\left(x-i 0^{+}\right)-\sigma\left(x+i 0^{+}\right)}{2 \pi i\left|B(x)-\sigma\left(x-i 0^{+}\right) D(x)\right|^{2}} .
$$

Proof. The inversion formula (6.4) implies

$$
\frac{d \psi(x ; \sigma)}{d x}=\frac{1}{2 \pi i}\left[\frac{A(x)-\sigma\left(x-i 0^{+}\right) C(x)}{B(x)-\sigma\left(x-i 0^{+}\right) D(x)}-\frac{A(x)-\sigma\left(x+i 0^{+}\right) C(x)}{B(x)-\sigma\left(x+i 0^{+}\right) D(x)}\right]
$$

which equals the right-hand side of (7.8), since $A(z) D(z)-B(z) C(z)=1$ for all $z$.

To illustrate the usefulness of Theorems 7.2 and 7.3 we consider two examples. In the first example we choose $\sigma(z)$ in Theorem 7.2 as

$$
g(z):=\frac{1}{2}\left[b z+c-\frac{1}{b z+c}\right]
$$


for real $c$ and $b \geq 0$, so that $\sigma$ in $(6.1)$ is $\sigma_{1}(z)$,

$$
\sigma_{1}(z)=-B\left(\left(b z+c-(b z+c)^{-1}\right) / 2\right) / D\left(\left(b z+c-(b z+c)^{-1}\right) / 2\right) .
$$

In the second example we choose the $\sigma(z)$ to be $\sigma_{2}(z)$,

$$
\sigma_{2}(z):=-B(b \sqrt{z}) / D(b \sqrt{z})=\frac{\left(q^{2} ; q^{2}\right)_{\infty}}{\left(q ; q^{2}\right)_{\infty}} f(b \sqrt{z}), \quad b>0 .
$$

Theorem 7.4. Let $F(z)$ denote either side of (6.1). If $F$ has an isolated pole singularity at $z=u$ then

$$
\operatorname{Res}[F(z) \text { at } z=u]=\operatorname{Res}\left[\frac{1}{D(z)[B(z)-\sigma(z) D(z)]} \text { at } z=u\right] \text {. }
$$

Proof. At a $z=u$ pole of $F(z), \sigma(u)=B(u) / D(u)$, so that

$$
A(u)-\sigma(u) C(u)=\frac{A(u) D(u)-B(u) C(u)}{D(u)}=\frac{1}{D(u)},
$$

and the theorem follows.

Theorems 7.3 and 7.4 show that the explicit form of $A(z)$ and $C(z)$ may not enter directly into the computation of the isolated masses or the absolutely continuous component of $d \psi$. It is important, however, to note that the qualitative behavior of $A(z)$ and $C(z)$ influence the nature of the spectrum and the spectral measure $d \psi$.

Example 1. $\sigma=\sigma_{1}$. Apply (6.25) with $a=e^{-\eta}=b z+c, b \geq 0$, and $c$ is real. The zeros of $B(\sinh \xi) D(\sinh \eta)-B(\sinh \eta) D(\sinh \xi)$ are still given by (6.27) except for the fact that $a$ is now $b x+c$. Thus the zeros of the aforementioned quantity are solutions of

$$
x=\frac{1}{2}\left[q^{-n}(b x+c)^{-1}-q^{n}(b x+c)\right],
$$

that is, they satisfy

$$
b\left(2+b q^{n}\right) x^{2}+2 c\left(1+b q^{n}\right) x+q^{n} c^{2}-q^{-n}=0 .
$$

Let $x_{n, 1}, x_{n, 2}$ be the roots of the above equation, so that

$$
\begin{aligned}
& x_{n, 1}=\frac{-c\left(1+b q^{n}\right)-\sqrt{c^{2}+2 b q^{-n}+b^{2}}}{b\left(2+b q^{n}\right)}, \\
& x_{n, 2}=\frac{-c\left(1+b q^{n}\right)+\sqrt{c^{2}+2 b q^{-n}+b^{2}}}{b\left(2+b q^{n}\right)} .
\end{aligned}
$$

The extremal measures correspond to the case $b=0$. It is clear from (7.11) that $x_{n, 1} \rightarrow-\infty$ as $b \rightarrow 0$ but $x_{n, 2} \rightarrow\left(q^{-n} / c-q^{n} c\right) / 2$ as $b \rightarrow 0$. Thus as $b \rightarrow 0$ the masses located at $x_{n, 1}$ disappear. Next we compute the residues. When $a$ was a constant the singular term in the Stieltjes transform of $d \psi$ in a neighborhood of $x=x_{n, 1}$, for $n \geq 0$ was $1 /\left[1-2 a q^{n} \sinh \xi-a^{2} q^{2 n}\right]$. The 
contribution of this factor to the residue is

$$
\lim _{x \rightarrow x_{n, j}} \frac{x-x_{n, j}}{1-2 a q^{n} \sinh \xi-a^{2} q^{2 n}},
$$

that is $-1 /\left(2 a q^{n}\right)$. When $a$ is $b x+c$ the same factor contributes

$$
\lim _{x \rightarrow x_{n, j}} \frac{x-x_{n, j}}{1-2(b x+c) q^{n} \sinh \xi-(b x+c)^{2} q^{2 n}},
$$

that is

$$
\frac{-q^{-n} / 2}{2 b x+c+q^{n} b(b x+c)}=-\frac{\left(2 a q^{n}\right)^{-1}}{2+b q^{n}-c /(b x+c)}=\frac{-q^{-n}}{2 a} \frac{1}{2+b q^{n}-c / a} .
$$

Therefore the residue at $x=x_{n, j}$ is $m_{n} /\left[2+b q^{n}-c / a\right]$ where $m_{n}$ is given by (6.30) and $a$ must be replaced by $b x_{n, j}+c$. When $n<0$, the singular factor in the Stieltjes transform in the neighborhood of $x_{n, j}$ comes from

$$
1 /\left[1+2 q^{n} a^{-1} \sinh \xi+q^{2 n} a^{-2}\right] .
$$

This contributes

$$
\lim _{x \rightarrow x_{n, j}} \frac{x-x_{n, j}}{1+2 q^{n} a^{-1} \sinh \xi-q^{2 n} a^{-2}}
$$

to the residue at $x=x_{n, j}$. When $a$ was a constant, this contributed $a\left(2 q^{n}\right)^{-1}$ but now it contributes the value of the above limit, that is

$$
\frac{a}{2 q^{n}} \frac{b x_{n, j}+c}{\left(2+b q^{n}\right)\left(b x_{n, j}+c\right)-c} \text {. }
$$

Thus for all $n$ the mass at $x_{n, j}$ is

$$
\begin{aligned}
& m_{n, j}(b, c)= \frac{\left(b x_{n, j}+c\right)^{4 n+1}}{\left(2+b q^{n}\right)\left(b x_{n, j}+c\right)-c} \\
& \times \frac{q^{n(2 n-1)}\left[1+\left(b x_{n, j}+c\right)^{2} q^{2 n}\right]}{\left(-\left(b x_{n, j}+c\right)^{2},-q\left(b x_{n, j}+c\right)^{-2}, q ; q\right)_{\infty}}, \\
& n=0, \pm 1, \pm 2, \ldots, j=1,2 .
\end{aligned}
$$

Theorem 7.5 (A generalization of the ${ }_{6} \psi_{6}$ sum). Let $\left\{x_{n, j} \mid n=0, \pm 1, \pm 2\right.$, $\ldots ; j=1,2\}$ be as in (7.13) and define $\xi_{n, j}$ through $x_{n, j}=\sinh \xi_{n, j}$. If $\left|t_{1} t_{2} t_{3} t_{4}\right|<q^{3}, b \geq 0$, and $c$ is real, then

$$
\begin{aligned}
\sum_{j=1}^{2} \sum_{n=-\infty}^{\infty} & \frac{\prod_{k=1}^{4}\left(t_{k} e^{-\xi_{n, j}},-t_{k} e^{\xi_{n, j}} ; q\right)_{\infty}}{\left(-\left(b x_{n, j}+c\right)^{2},-q\left(b x_{n, j}+c\right)^{-2} ; q\right)_{\infty}} \\
& \times \frac{\left(b x_{n, j}+c\right)^{4 n+1} q^{n(2 n-1)}\left[1+q^{2 n}\left(b x_{n, j}+c\right)^{2}\right]}{\left(2+b q^{n}\right)\left(b x_{n, j}+c\right)-c} \\
= & \frac{(q ; q)_{\infty}}{\left(t_{1} t_{2} t_{3} t_{4} q^{-3} ; q\right)_{\infty}} \prod_{1 \leq r<s \leq 4}\left(-t_{r} t_{s} / q ; q\right)_{\infty} .
\end{aligned}
$$

Proof. The identity (7.16) is (3.8) when $d \psi$ is the discrete measure in Example 1 , provided that the bilateral infinite series in (7.16) converges. If $b=0,(7.16)$ is the ${ }_{6} \psi_{6}$ sum, as noted earlier. The convergence of the series is established as follows. First observe that for large positive $n, x_{n, 1}<0<x_{n, 2}$ and as 
$n \rightarrow+\infty, x_{n, 1} \approx-q^{-n / 2} / \sqrt{2 b}, x_{n, 2} \approx q^{n / 2} / \sqrt{2 b}$. With $x_{n, j}=\sinh \xi_{n, j}$, we find $e^{\xi_{n, 1}} \approx-q^{-n / 2} / \sqrt{\frac{2}{b}}$ and $e^{\xi_{n, 2}} \approx q^{-n / 2} / \sqrt{\frac{2}{b}}$. This observation then implies that when $j=1$ and $n \rightarrow-\infty$ the summand in (7.16) is

$$
\begin{aligned}
& O\left(\frac{\prod_{k=1}^{4}\left(-t_{k} q^{-n / 2} \sqrt{2 / b} ; q\right)_{[n / 2]}}{\left(-b q^{-n} / 2 ; q\right)_{n}}\left(b q^{-n} / 2\right)^{2 n} q^{n(2 n-1)} q^{2 n}\left(q^{-n / 2} \sqrt{b / 2}\right)^{3}\right) \\
& \quad=O\left(\left(t_{1} t_{2} t_{3} t_{4} q^{-3}\right)^{n / 2} .\right.
\end{aligned}
$$

One can similarly handle the case $j=2$. On the other hand $x_{n, j} \rightarrow-c / b$ as $n \rightarrow-\infty$. Thus the bilateral infinite series converges when $j=1,2$, provided that $\left|t_{1} t_{2} t_{3} t_{4}\right|<q^{3}$. This completes the proof.

We shall have more to say about (7.16) in Section 9.

Example 2. $\sigma=\sigma_{2}$, see (7.11). In this case

$$
\int_{-\infty}^{\infty} \frac{d \psi\left(t ; \sigma_{2}\right)}{z-t}=\frac{A(z) D(b \sqrt{z})+C(z) B(b \sqrt{z})}{B(z) D(b \sqrt{z})+D(z) B(b \sqrt{z})} .
$$

Using (6.25) with $\sinh \eta=-b \sqrt{z}, b>0$ we get

$B(z) D(b \sqrt{z})+D(z) B(b \sqrt{z})=\frac{e^{-\zeta}}{2(q ; q)_{\infty}}\left(e^{\xi+\zeta},-e^{\zeta-\xi}, q e^{-\xi-\zeta},-q e^{\xi-\zeta} ; q\right)_{\infty}$,

where

$$
z=\sinh \xi, \quad \sinh \zeta=b \sqrt{z} .
$$

We will measure the argument of a complex number $z$ as $-\pi<\arg z \leq \pi$. It is clear from (7.5) and (7.18) that $\sigma_{2}(z)$ is single-valued across the positive real axis and

$$
\sigma_{2}\left(x-i 0^{+}\right)-\sigma_{2}\left(x+i 0^{+}\right)=\sigma_{2}\left(|x| e^{-i \pi}\right)-\sigma_{2}\left(|x| e^{i \pi}\right)=\frac{2 i\left(q ; q^{2}\right)_{\infty}}{b \sqrt{|x|}\left(q^{2} ; q^{2}\right)_{\infty}} .
$$

For $x<0$ the derivative of $\psi$ is

$$
\begin{aligned}
& \frac{d \psi\left(x ; \sigma_{2}\right)}{d x} \\
& =\frac{\left(q ; q^{2}\right)_{\infty}\left|\left(q^{2} e^{2 \zeta}, q^{2} e^{-2 \zeta} ; q^{2}\right)_{\infty}\right|^{2}}{\pi b \sqrt{|x|}\left(q^{2} ; q^{2}\right)_{\infty}\left(b^{2}+|x|\right)\left|\left(q e^{\xi+\zeta},-q e^{\zeta-\xi}, q e^{-\zeta-\zeta},-q e^{\xi-\zeta} ; q\right)_{\infty}\right|^{2}} .
\end{aligned}
$$

From the general theory of moment problems we know that the poles of the right side of (7.17) are real and simple. If $z=u$ is such a pole then the corresponding value of $\zeta$ will be real if $z \geq 0$ but will be purely imaginary if $z<0$. It is clear, however, from (7.18) that $B(z) D(b \sqrt{z})+D(z) B(b \sqrt{z}) \neq 0$ if $\xi$ is real and $\zeta$ is purely imaginary. Therefore the zeros of the right-hand side of (7.18) are nonnegative, so $\xi \geq 0$ and $\zeta$ is real. This shows that the 
zeros coincide with the roots of

$$
e^{\xi+\zeta}=q^{-n}, \quad n=0, \pm 1, \pm 2, \ldots
$$

Obviously $e^{\xi+\zeta}=q^{-n}$ if and only if

$$
\left(z+\sqrt{z^{2}+1}\right)\left(b \sqrt{z}+\sqrt{1+b^{2} z}\right)=q^{-n}, \quad \frac{1}{1+a^{2}} n=0, \pm 1, \pm 2, \ldots
$$

For $n>0$, equation (7.23) has a unique solution $z=x_{n}$ since its right-hand side strictly increases from 1 to $\infty$ as $z$ increases from 0 to $\infty$. Equation (7.23) has no solutions if $n<0$ for the same reason. When $z=x_{n}$, let $\xi_{n}$ and $\zeta_{n}$ be the corresponding values of $\xi$ and $\zeta$; respectively. Thus $e^{\zeta_{n}}=q^{n} e^{-\xi_{n}}$. We apply Theorem 7.4 and find that the residue of the right-hand side of (7.17) at $z=x_{n}, n>0$, is

$$
\lim _{z \rightarrow x_{n}} \frac{2\left(z-x_{n}\right)(q ; q)_{\infty} e^{\zeta_{n}} D(b \sqrt{z}) / D(z)}{\left(q^{-n} ; q\right)_{n}\left(1-q^{n} e^{\xi+\zeta}\right)\left(q, q^{n+1},-q^{-n} e^{-2 \xi_{n}},-q^{n+1} e^{2 \xi_{n}} ; q\right)_{\infty}} .
$$

In view of the following alternate expression for $D(z)$,

$$
D(z)=\frac{e^{\xi}\left(q^{2} e^{2 \xi}, e^{-2 \xi} ; q^{2}\right)_{\infty}}{2(q ; q)_{\infty}}
$$

the above limit is

$$
\begin{aligned}
\lim _{z \rightarrow x_{n}} & \frac{z-x_{n}}{1-q^{n} e^{\xi+\zeta}} \\
& \times \frac{2 \zeta^{\zeta_{n}}(q ; q)_{\infty} e^{\zeta_{n}-\xi_{n}}\left(q^{2-2 n} e^{-2 \xi_{n}}, q^{2 n} e^{2 \xi_{n}} ; q^{2}\right)_{\infty}}{\left(q^{n+1},-q^{-n} e^{-2 \xi_{n}}, q,-q^{n+1} e^{2 \xi_{n}} ; q\right)_{\infty}\left(q^{-n} ; q\right)_{n}\left(q^{2} e^{2 \xi_{n}}, e^{-2 \xi_{n}} ; q^{2}\right)_{\infty}} .
\end{aligned}
$$

After some simplifications we find that the point mass at $x=x_{n}$, being the residue at $z=x_{n}$, is given by

$$
M_{n}:=\text { Mass at } x_{n}=\frac{4 \cosh \xi_{n}}{A_{n}\left(q,-e^{-2 \xi_{n}},-e^{2 \xi_{n}} ; q\right)_{\infty}},
$$

with $A_{n}=\left.(d \xi / d z+d \zeta / d z)\right|_{x=x_{n}}$. Thus

$$
A_{n}=\frac{1}{\cosh \xi_{n}}+\frac{b^{2}}{\sinh \left(2 \zeta_{n}\right)} .
$$

If $n=0$ then $z=0$ is the only solution to (7.23). On the other hand $z=0$ is not an isolated singularity of the right-hand side of (7.17). We have not been able to show that $x=0$ does not support a discrete mass. We strongly believe that $x=0$ is not a discrete mass point. One reason for this belief is that if we formally substitute $n=0$ in $A_{n}$ then we find $A_{0} \rightarrow \infty$, hence $M_{0}=0$.

We now discuss the spectral measures corresponding to the choice of $\sigma$ in (6.35). For $\zeta$ in the open upper half plane define

$$
\sigma(z):=-B(\zeta) / D(\zeta), \Im z>0, \quad \sigma(\bar{z})=\overline{\sigma(z)} .
$$


The above representation is an alternate representation for $\sigma(z)$ of (6.35). The mapping $z=\sinh \eta$ is a one-to-one mapping of the strip

$$
D:=\{\eta: 0<\Im \eta<\pi / 2\} \cup\{\eta: \Im \eta=\pi / 2, \operatorname{Re} \eta \leq 0\},
$$

onto the half plane $\Im z>0$. Taking into account that $B(z)$ is an even function and $D(z)$ is an odd function we then rewrite $\sigma(z)$ as

$$
\sigma(z):=B(-\sinh \eta) / D(-\sinh \eta), \Im z>0, \quad \sigma(\bar{z})=\overline{\sigma(z)} .
$$

If we denote the corresponding spectral measure by $d \mu(t ; \eta)$ then

$$
\frac{A(z) D(\sinh \eta)+C(z) B(\sinh \eta)}{B(z) D(\sinh \eta)+D(z) B(\sinh \eta)}=\int_{-\infty}^{\infty} \frac{d \mu(t ; \eta)}{z-t}, \quad \eta \in D .
$$

To find $\mu$ first observe the left-hand side of (7.28) has no poles as can be seen from (6.25). Thus $d \mu$ is absolutely continuous. In addition (7.8) yields

$$
\frac{d \mu(x ; \eta)}{d x}=\frac{B(\sinh \eta) D(\sinh \bar{\eta})-B(\sinh \bar{\eta}) D(\sinh \eta)}{2 \pi i|B(x) D(-\sinh \eta)-D(x) B(-\sinh \eta)|^{2}} .
$$

After applying (6.25) and some simplifications we obtain

$$
\begin{aligned}
\frac{d \mu(x ; \eta)}{d x} & =\frac{e^{2 \eta_{1}} \sin \eta_{2} \cosh \eta_{1}\left(q,-q e^{2 \eta_{1}},-q e^{-2 \eta_{1}}\right)_{\infty}\left|\left(q e^{2 i \eta_{2}}\right)_{\infty}\right|^{2}}{\pi\left|\left(e^{\xi+\eta},-e^{\eta-\xi},-q e^{\xi-\eta}, q e^{-\xi-\eta} ; q\right)_{\infty}\right|^{2}}, \\
x & =\sinh \xi, \eta=\eta_{1}+i \eta_{2} .
\end{aligned}
$$

Therefore we have established the following $q$-beta integral

$$
\begin{aligned}
& \int_{-\infty}^{\infty} \frac{\prod_{j=1}^{4}\left(-t_{j} e^{\xi}, t_{j} e^{-\xi}\right)_{\infty}}{\left|\left(e^{\xi+\eta},-e^{\eta-\xi},-q e^{\xi-\eta}, q e^{-\xi-\eta}\right)_{\infty}\right|^{2}} \cosh \xi d \xi \\
& \quad=\frac{\pi e^{-2 \eta_{1}} \prod_{1 \leq j<k \leq 4}\left(-t_{j} t_{k} / q\right)_{\infty}}{\sin \eta_{2} \cosh \eta_{1}\left(q, t_{1} t_{2} t_{3} t_{4} q^{-3},-q e^{2 \eta_{1}},-q e^{-2 \eta_{1}}\right)_{\infty}\left|\left(q e^{2 i \eta_{2}}\right)_{\infty}\right|^{2}} .
\end{aligned}
$$

The $q$-beta integral (7.30) is new.

Observe that as $\eta_{2} \rightarrow 0$ the integral in (7.30) diverges and the right-hand side of (7.30) also diverges. If we multiply (7.30) by $\sin \eta_{2}$ then let $\eta_{2} \rightarrow 0$ then the left-hand side will become a ${ }_{6} \psi_{6}$ function while the right-hand side will give its sum. This is expected since the case when $\sigma$ is a real constant leads to the ${ }_{6} \psi_{6}$ sum.

Askey [10] proved that the polynomials

$$
p_{n}(\sinh \xi)={ }_{4} \varphi_{3}\left(\begin{array}{c}
q^{-n}, q^{n+3} / t_{1} t_{2} t_{3} t_{4}, q e^{\xi} / t_{1},-q e^{-\xi} / t_{1} \\
-q^{2} / t_{1} t_{2},-q^{2} t_{1} t_{3},-q^{2} / t_{1} t_{4}
\end{array} \mid q, q\right)
$$

satisfy the orthogonality relation

$$
\int_{-\infty}^{\infty} \frac{p_{m}(\sinh \xi) p_{n}(\sinh \xi) \prod_{j=1}^{4}\left(t_{j} e^{-\xi},-t_{j} e^{\xi}\right)_{\infty}}{(-\ln q)\left(q,-q e^{2 \xi},-q e^{-2 \xi}\right)_{\infty}} d \xi=M_{n} \delta_{m, n}
$$


$m, n=0,1, \ldots, N$, with

$$
\begin{aligned}
M_{n}= & \frac{\left(q^{-n}, q^{n+3} / t_{1} t_{2} t_{3} t_{4},-q^{2} / t_{2} t_{3},-q^{2} / t_{2} t_{4}\right)_{n}}{\left(q^{4} / t_{1} t_{2} t_{3} t_{4},-q^{2} / t_{1} t_{3},-q^{2} / t_{1} t_{4}\right)_{n}} \\
& \times\left(q t_{2}^{2} / t_{1}^{2}\right)^{n}\left[\prod_{1 \leq j<k \leq 4}\left(-t_{j} t_{k} / q\right)_{\infty}\right] /\left(t_{1} t_{2} t_{3} t_{4} q^{-3}\right)_{\infty}, \\
& \left|t_{1} t_{2} t_{3} t_{4}\right|<q^{3+2 N} .
\end{aligned}
$$

It is clear that the orthogonality relation (7.32) only uses the moments of the measure involved. This implies

$$
\int_{-\infty}^{\infty} p_{m}(\sinh \xi) p_{n}(\sinh \xi) \prod_{j=1}^{4}\left(t_{j} e^{-\xi},-t_{j} e^{\xi}\right)_{\infty} d \psi(x)=M_{n} \delta_{m, n}
$$

where $d \psi$ is a probability measure and $\left\{h_{n}(x \mid q)\right\}$ are orthogonal with respect to $d \psi$. The restrictions on $m, n$ are $0 \leq m, n \leq N$ such that the left-hand side of (7.35) exists. It is interesting to note that (7.34) must be satisfied due to the positivity condition on the coefficients in the three term recurrence relation, see [16], [17], and [25]. It is not difficult to see that (7.34) is the only restriction required when $d \psi$ is an extremal measure as given in (6.27), (6.31). Askey's proof of (7.32) starts from (7.3) and is similar to the proof of orthogonality of the Askey-Wilson polynomials as given by Askey and Wilson in [17].

\section{A ${ }_{6} \psi_{6}$ SUM WITH INFINITELY MANY PARAMETERS}

Let $d \rho(x)$ be a discrete probability measure with a finite or infinite support. Define $g(z)$ by

$$
g(z):=b z+c-\int_{-\infty}^{\infty} \frac{d \rho(t)}{z-t}, \quad b \geq 0, c \in R .
$$

It is clear from (8.1) that $g(z)$ maps the open upper half plane into itself and that $g(z)$ is meromorphic with only real poles. Choose $\sigma(z)$ in $(6.1)$ as

$$
\sigma(z):=-B(g(z)) / D(g(z)) \text {. }
$$

Let us denote either side of $(6.1)$ by $F(z ; \sigma)$, that is

$$
F(z ; \sigma)=\frac{A(z) D(g(z))+B(g(z)) C(z)}{B(z) D(g(z))+B(g(z)) D(z)}=\int_{-\infty}^{\infty} \frac{d \psi(t ; \sigma)}{z-t}
$$

Thus $F(z ; \sigma)$ is meromorphic and its poles are solutions of

$$
a e^{\xi}=q^{n}, \quad n=0, \pm 1, \ldots,
$$

where

$$
z=\sinh \xi, g(z)=\sinh \eta, a=e^{\eta}
$$


In other words the poles of $F(z ; \sigma)$ are the roots of

$$
\left[g(x)+\sqrt{1+g^{2}(x)}\right]\left[x+\sqrt{1+x^{2}}\right]=q^{n}, \quad n=0, \pm 1, \pm 2, \ldots
$$

The representation (8.1) shows that when $x$ is real, the graph of $g(x)$ consists of branches, and $g^{\prime}(x)>0$ on each branch. Thus $g$ strictly increases with $x$ on any branch of $g$. Therefore for any fixed $n$ (8.6) has solutions $\left\{x_{n, j}\right\}$, where $j$ enumerates the roots for a given $n$. We shall arrange the $x$ 's so that

$$
x_{n, j}<x_{n, j+1} \text {. }
$$

Theorem 8.1 (A ${ }_{6} \psi_{6}$ sum with finitely many parameters). Assume that $g(z)$ is a rational function and let $x_{n, j}$ be as above. Define $\eta_{n, j}$ and $\xi_{n, j}$ by $g\left(x_{n, j}\right)=$ $\sinh \eta_{n, j}$ and $x_{n, j}=\sinh \xi_{n, j}$. If $\left|t_{j}\right|<1,1 \leq j \leq 4$, and $\left|t_{1} t_{2} t_{3} t_{4}\right|<q^{3}$ then

$$
\begin{aligned}
\sum_{j} \sum_{n=-\infty}^{\infty} \frac{a_{n, j}^{4 n} q^{n(2 n-1)}\left(1+q^{2 n} a_{n, j}^{2}\right)}{\left(-a_{n, j}^{2},-q / a_{n, j}^{2} ; q\right)_{\infty}} \frac{\cosh \eta_{n, j}}{\cosh \eta_{n, j}+g^{\prime}\left(x_{n, j}\right) \cosh \xi_{n, j}} \\
\quad \times \prod_{m=1}^{4}\left(t_{m} e^{-\xi_{n, j}},-t_{m} e^{\xi_{n, j}} ; q\right)_{\infty} \\
=\frac{(q ; q)_{\infty}}{\left(t_{1} t_{2} t_{3} t_{4} q^{-3} ; q\right)_{\infty}} \prod_{1 \leq r<s \leq 4}\left(-t_{r} t_{s} / q ; q\right)_{\infty} .
\end{aligned}
$$

The proof consists of two parts. The first part involves computing the residues of $F(z ; \sigma)$ while the second part establishes the convergence of the series in (8.8). The second part of the proof will be divided further into two cases depending on whether $b>0$ or $b=0$.

Proof, Part 1. With $\sigma(z)$ as in (8.2) we apply Theorem 7.4 and obtain

$$
\begin{aligned}
\operatorname{Res}\left[\frac{A(z)-\sigma(z) C(z)}{B(z)-\sigma(z) D(z)}\right] & =\operatorname{Res}\left[\frac{1 / D(z)}{B(z)-\sigma(z) D(z)}\right] \\
& =\operatorname{Res}\left[\frac{D(g(z)) / D(z)}{B(z) D(g(z))+D(z) B(g(z))}\right] \\
& =\operatorname{Res}\left[\frac{D(-g(z)) / D(z)}{B(z) D(-g(z))+D(z) B(-g(z))}\right] .
\end{aligned}
$$

From that above equality, (5.32), (5.36), and (6.25) we find that $F(z ; \sigma)$ and

$$
\frac{2 a(q ; q)_{\infty} \sinh \eta\left(q^{2} e^{2 \eta}, q^{2} e^{-2 \eta} ; q^{2}\right)_{\infty}}{\sinh \xi\left(a e^{\xi}, a e^{-\xi}, q e-\xi / a,-q e^{\xi / a} ; q\right)_{\infty}\left(q^{2} e^{2 \xi}, q^{2} e^{-2 \xi} ; q^{2}\right)_{\infty}}
$$

have the same residues at the poles of $F(z ; \sigma)$. We have already calculated the residues of the expression (8.9) when $a$ and $\eta$ were constants. Now $a$ depends on $z$. When $n>0$ the only difference in the new calculation is that when computing

$$
\lim _{z \rightarrow x_{n, k}} \frac{z-x_{n, k}}{1-q^{n} e^{-\xi} / a}
$$


we should take into account that $a$ is no longer a constant. When $a$ were a constant, the above limit came out to be $\cosh \xi$ but now it becomes $\frac{\cosh \xi}{1+a^{-1} d a / d \xi}$. Since $a=g(z)+\sqrt{1+g^{2}(z)}$, a calculation gives

$$
\frac{d a}{d \xi}=\frac{a g^{\prime}(z) \cosh \xi}{\cosh \eta}
$$

Thus we divide the masses in (6.30) by $1+a^{-1} \frac{d a}{d \xi}$, that is $1+\frac{g^{\prime}\left(x_{n, j}\right) \cosh \xi_{n, j}}{\cosh \eta_{n, j}}$. Similarly we see that the masses in (6.26) must also be multiplied by the same factor when $n \leq 0$. Thus Theorem 8.1 will follow if we prove that the double series on left-hand side of $(8.8)$ is absolutely convergent.

Proof, Part 2. The case $b>0$. Let $s_{1}<s_{2}<\cdots<s_{k}$ be the poles of $g(z)$ and define $s_{0}$ and $s_{k+1}$ to be $-\infty$ and $\infty$; respectively. For every $r$ y equation (8.6) has a unique solution in $\left(s_{r}, s_{r+1}\right)$ and

$$
-\infty=s_{0}<x_{n, 0}<s_{1}<x_{n, 1}<\cdots<s_{k}<x_{n, k}<s_{k+1}=+\infty .
$$

It is clear from (8.6) that $x_{n, j}$ is a strictly decreasing function of $n$ for every fixed $j$. Furthermore $x_{n, j} \rightarrow s_{j}+0^{+}$as $n \rightarrow \infty$, for all $j$. On the other hand $x_{n, j} \rightarrow s_{j+1}$ as $n \rightarrow-\infty$, for all $j$. As $n \rightarrow \infty$ we see from (8.5) and (8.6) that $a_{n, j} \approx q^{n} /\left[s_{j}+\sqrt{1+s_{j}^{2}}\right]$. Hence, for $j>0$, we have

$$
\left(-q / a_{n, j}^{2} ; q\right)_{\infty}=O\left(\left(-q / a_{n, j}^{2} ; q\right)_{2 n}\right)=O\left(q^{n(1-2 n)}\left(s_{j}+\sqrt{1+s_{j}^{2}}\right)^{2 n}\right) .
$$

The left side of (8.8) is of the form

$$
\sum_{j=0}^{k} \sum_{n=-\infty}^{\infty} C_{j, n} .
$$

The above analysis shows that $\sum_{j=1}^{k} \sum_{n=0}^{\infty} C_{j, n}$ converges absolutely. The idea behind the proof of Theorem 7.5 will establish the absolute convergence of $\sum_{n=0}^{\infty} C_{0, n}$. Similarly as $n \rightarrow-\infty$,

$$
a_{n, j} \approx q^{n} /\left[s_{j}+\sqrt{1+s_{j}^{2}}\right] \text { for } j<k .
$$

This implies the convergence of $\sum_{j=0}^{k-1} \sum_{n=0}^{\infty} C_{j,-n}$. Finally $\sum_{n=0}^{\infty} C_{0,-n}$ can be proved to converge absolutely by an argument similar out proof of Theorem 7.5. This completes the proof of Case 1.

Proof, Part 2. The case $b=0$. In this case we lose one of the mass points because $g(x) \rightarrow c$ as $x \rightarrow \pm \infty$. The rest of the proof is identical to the proof in the first case.

Theorem 8.2 (A ${ }_{6} \psi_{6}$ sum with infinitely many parameters). Assume that $\rho$ in (8.1) has infinitely many points of increase. Let $x_{n, j}, \eta_{n, j}$ and $\xi_{n, j}$ be as in 
Theorem 8.1. If $\left|t_{j}\right|<1,1 \leq j \leq 4$, and $\left|t_{1} t_{2} t_{3} t_{4}\right|<q^{3}$ then

$$
\sum_{j} \sum_{n=-\infty}^{\infty} \frac{a_{n, j}^{4 n} q^{n(2 n-1)}\left(1+q^{2 n} a_{n, j}^{2}\right)}{\left(-a_{n, j}^{2},-q / a_{n, j}^{2} ; q\right)_{\infty}} \frac{\cosh \eta_{n, j}}{\cosh \eta_{n, j}+g^{\prime}\left(x_{n, j}\right) \cosh \xi_{n, j}}
$$

$$
\begin{aligned}
& \times \prod_{m=1}^{4}\left(t_{m} e^{-\xi_{n, j}},-t_{m} e^{\xi_{n, j}} ; q\right)_{\infty} \\
= & \frac{(q ; q)_{\infty}}{\left(t_{1} t_{2} t_{3} t_{4} q^{-3} ; q\right)_{\infty}} \prod_{1 \leq r<s \leq 4}\left(-t_{r} t_{s} / q ; q\right)_{\infty},
\end{aligned}
$$

provided that the double series in (8.11) converges.

To prove Theorem 8.2 we proceed as in the proof of Part 1 of Theorem 8.1, then use the Perron-Stieltjes inversion formula. The details are straightforward and will be omitted.

We believe that Theorem 8.2 is valid without assuming that the double series in $(8.11)$ converges. It is very likely that the remaining assumptions imply the convergence of the double series in (8.11).

\section{REMARKS}

We first give an alternate derivation of the extremal measures of Section 6 using only the quasiperiodicity of theta functions and Theorem 7.4. This approach has two advantages. First it may be applicable to other polynomials since it only assumes that $B(z)$ and $D(z)$ are quasiperiodic of the same period. Secondly it illustrates the usefulness of Theorem 7.4. It is worth pointing out that the only functions having a period and a real period are essentially theta functions, see Section 10 in [40].

Recall that with the parameterization (6.19), that is

$$
\sigma=B(\sinh \eta) / D(\sinh \eta),
$$

the extremal measures are supported at the zeros of

$$
\Phi(z):=B(z) D(\sinh \eta)-D(z) B(\sinh \eta) .
$$

Thus $z=\sinh \eta$ is a mass point. Now assume that $B(z)$ and $D(z)$ are quasiperiodic functions satisfying

$$
B(\sinh (\xi+u))=f(\xi) B(\sinh \xi), \quad D(\sinh (\xi+u))=f(\xi) D(\sinh \xi) .
$$

If $B(z)$ and $D(z)$ are theta functions $\vartheta(i \sinh \xi ; q)$ then $f(\xi)=-q^{-1} e^{2 \xi}$ and $u=-\ln q$ [52]. From (9.2) we see that $\Phi(\sinh \xi)$ vanishes at $\xi=\eta+n u, n=$ $0, \pm 1, \ldots$, and the corresponding values of $z$; say $\left\{x_{n}\right\}_{-\infty}^{\infty}$, are

$$
x_{n}=\frac{1}{2}\left(e^{n u} e^{\eta}-e^{-n u} e^{-\eta}\right)
$$

When $u=-\ln q$ equation (9.3) is (6.27). In order to show that all the zeros of (9.2) are given by (7.3) we only need to show that there exists a unique point of the form (9.3) in each open interval formed by two consecutive zeros of $B(z)$. This is the case since the graph of $D(x) / B(x)$ resembles the graph of 
$\tan x$ and $D(x) / B(x)=a$ has a unique solution in any open interval formed by two consecutive poles of $D(z) / B(z)$. Furthermore the masses at $x_{n}$ can be found from the residue of the Stieltjes transform of the extremal measure at $x_{0}$ $(=\sinh \eta)$ in the following manner. First apply the quasiperiodicity property (9.2) to (9.1) and obtain

$$
\Phi(\sinh (\xi+n u))=\left[\prod_{j=0}^{n-1} f(\xi+j u)\right] \Phi(\sinh \xi) .
$$

Thus Theorem 7.4 shows that the mass at $x_{n}$ is

$$
\begin{aligned}
\operatorname{Res} & {\left[\frac{D(\sinh \eta)}{D(z) \Phi(z)} \text { at } z=x_{n}\right] } \\
& =\cosh (\eta+n u) \operatorname{Res}\left[\frac{D(\sinh \eta) / D(\sinh (\eta+n u))}{\Phi(\sinh (\xi+n u))} \text { at } \xi=\eta\right] . \\
& =\frac{\cosh (\eta+n u)}{\prod_{j=0}^{n-1} f^{2}(\eta+j u)} \operatorname{Res}\left[\frac{D(\sinh \eta)}{D(\sinh \xi) \Phi(\sinh \xi)} \text { at } \xi=\eta\right] \\
& =\frac{\cosh (\eta+n u)}{\cosh \eta \prod_{j=0}^{n-1} f^{2}(\eta+j u)} m_{0},
\end{aligned}
$$

where $m_{0}$ is the mass at $x=\sinh \eta$. Let $m_{n}$ be the mass at. $x_{n}$. Thus we have proved

$$
m_{n}=\frac{\cosh (\eta+n u)}{\cosh \eta \prod_{j=0}^{n-1} f^{2}(\eta+j u)} m_{0} .
$$

To calculate $m_{0}$ we either have to compute the residue at $z=\sinh \eta$ directly from Theorem 7.4 or use the normalization $\sum_{-\infty}^{\infty} m_{n}=1$. The latter approach leads to

$$
m_{0}=\cosh \eta /\left[\sum_{-\infty}^{\infty} \cosh (\eta+n u) / \prod_{j=0}^{n-1} f^{2}(\eta+j u)\right] .
$$

In the moment problem associated with $q^{-1}$-Hermite polynomials $u=-\ln q$ and $f(\xi)=-q^{-1} e^{2 \xi}$. In this case formula (9.5) is (6.30) and we have found an alternate and simpler derivation of (6.30).

We now explore some of the consequences of our explicit computation of the Stieltjes transforms of solutions of the $q^{-1}$-Hermite polynomials. In view of Theorem 7.2 we may choose $\sigma(z)$ as

$$
\sigma(z)=-B(\sinh \eta(z)) / D(\sinh \eta(z)),
$$

where $\sinh \eta=\sinh (\eta(z))$ maps the upper half plane into itself. This gives

$$
\frac{A(z) D(\sinh \eta)+C(z) B(\sinh \eta)}{B(z) D(\sinh \eta)+D(z) B(\sinh \eta)}=\int_{-\infty}^{\infty} \frac{d \psi(t ; \eta)}{z-t}
$$

From (6.25), (5.32), (5.36), and (5.45) we obtain

$$
\int_{-\infty}^{\infty} \frac{d \psi(t ; \eta)}{z-t}=2 N(\xi, \eta) / D(\xi, \eta)
$$


where

$$
\begin{aligned}
N(\xi, \eta):= & \frac{2\left(q^{2}, e^{2 \eta}, q^{2} e^{-2 \eta} ; q^{2}\right)_{\infty}}{\left(1 / q ; q^{2}\right)_{\infty}} \sinh \xi_{2} \phi_{1}\left(q e^{2 \xi}, q e^{-2 \xi} ; q^{3} ; q^{2}, q^{2}\right) \\
& -e^{\eta}\left(q e^{2 \eta}, q e^{-2 \eta} ; q^{2}\right)_{\infty}{ }_{2} \phi_{1}\left(q e^{2 \xi}, q e^{-2 \xi} ; q ; q^{2}, q^{2}\right)
\end{aligned}
$$

and

$$
D(\xi, \eta):=\left(e^{\xi+\eta},-e^{\eta-\xi},-q e^{\xi-\eta}, q e^{-\xi-\eta} ; q\right)_{\infty}
$$

When $\sigma$ is a real constant then $\eta$ is a real constant and (9.8) becomes

$$
\sum_{-\infty}^{\infty} \frac{e^{\eta} e^{4 n \eta} q^{2 n^{2}}\left(1+e^{2 \eta} q^{2 n}\right)}{\left(-e^{2 \eta},-q e^{-2 \eta} ; q\right)_{\infty}\left(1-2 q^{n} e^{\eta} \sinh \xi-q^{2 n} e^{2 \eta}\right)}=-N(\xi, \eta) / D(\xi, \eta) .
$$

With $a=e^{\eta}$ the identity (9.11) takes the form

$$
\begin{aligned}
& \sum_{-\infty}^{\infty} \frac{\left(i a q,-i a q, a e^{\xi},-a e^{-\xi} ; q\right)_{n}}{\left(i a,-i a,-a q e^{-\xi}, a q e^{\xi} ; q\right)_{n}} a^{4 n} q^{2 n^{2}} \\
& \quad=\frac{\left(-q a^{2},-q / a^{2} ; q\right)_{\infty}\left(a^{2}-2 a \sinh \xi-1\right)}{\left(a e^{\xi},-a e^{-\xi},-q e^{\xi} / a, q e^{-\xi} / a ; q\right)_{\infty}} \\
& \quad \times\left[\frac{2\left(q^{2}, a^{2}, q^{2} / a^{2} ; q^{2}\right)_{\infty}}{\left(1 / q ; q^{2}\right)_{\infty}} \sinh \xi_{2} \phi_{1}\left(q e^{2 \xi}, q e^{-2 \xi} ; q^{3} ; q^{2}, q^{2}\right)\right. \\
& \left.-a\left(q a^{2}, q / a^{2} ; q\right)_{\infty} \phi_{1}\left(q e^{2 \xi}, q e^{-2 \xi} ; q ; q^{2}, q^{2}\right)\right] .
\end{aligned}
$$

It is easy to see that the left side of formula (9.11) corresponds to the limiting case $b \rightarrow \infty, c \rightarrow \infty, d \rightarrow \infty, f \rightarrow \infty$ of

$$
{ }_{8} \psi_{8}\left(\begin{array}{c}
i q a,-i q a, a e^{\xi},-a e^{-\xi}, a b, a c, a d, a f \\
i a,-i a,-a q e^{-\xi}, a q e^{\xi},-a q / b,-a q / c,-a q / d, a q / f
\end{array} \mid q ; \frac{q^{2}}{b c d f}\right),
$$

where $a=e^{\eta}$. The ${ }_{8} \psi_{8}$ transformation, (III.38) in [25], expresses the ${ }_{8} \psi_{8}$ in (9.13) as a sum of two ${ }_{8} \phi_{7}$ functions. It is not clear how to establish (9.12) by performing the appropriate limits on the aforementioned ${ }_{8} \psi_{8}$ transformation. It is important however to to note that the ${ }_{8} \psi_{8}$ of (9.12) contains four additional free parameters, namely $b, c, d$, and $f$. This leads us to conjecture that the Stieltjes transform of

$$
\prod_{j=1}^{4}\left(-t_{j}\left(x+\sqrt{x^{2}+1}\right), t_{j}\left(\sqrt{x^{2}+1}-x\right)\right)_{\infty} d \psi(x ; \eta)
$$

when $\eta$ is a real constant is indeed a multiple of an ${ }_{8} \psi_{8}$ of the form (9.12) where $b, c, d, f$ are multiples of $1 / t_{1}, 1 / t_{2}, 1 / t_{3}, 1 / t_{4}$. The factor which is multiplied by the ${ }_{8} \psi_{8}$ is a quotient of products of infinite products. We do not know how to prove the latter assertion in general at this time. To see that 
this conjecture holds for extremal measures apply (6.27) and (6.30) to get, after some simplification,

$$
\begin{aligned}
\int_{-\infty}^{\infty} & \prod_{j=1}^{4}\left(-t_{j} e^{u}, t_{j} e^{-u} ; q\right)_{\infty} \frac{d \psi(\sinh u ;-\ln a)}{\sinh \xi-\sinh u} \\
= & \frac{2 a \prod_{j=1}^{4}\left(a t_{j},-t_{j} / a ; q\right)_{\infty}}{\left(a^{2}+2 a \sinh \xi-1\right)\left(-q a^{2},-q / a^{2} ; q\right)_{\infty}} \\
& \times{ }_{8} \psi_{8}\left(\begin{array}{c}
i q a,-i q a,-a q / t_{1},-a q / t_{2},-a q / t_{3},-a q / t_{4},-a e^{-\xi}, a e^{\xi} \\
i a,-i a, a t_{1}, a t_{2}, a t_{3}, a t_{4}, a q e^{\xi},-a q e^{-\xi}
\end{array} \mid\right. \\
& \left.q ; \frac{t_{1} t_{2} t_{3} t_{4}}{q^{2}}\right),
\end{aligned}
$$

One can derive a continuous analog of $(9.11)$ by choosing $\eta \in D, D$ as in (7.26) and use the measure $d \mu$ of (7.29). The result is

$$
\begin{aligned}
\int_{-\infty}^{\infty} & \frac{\cosh u}{\left|\left(e^{u+\eta},-e^{\eta-u},-q e^{u-\eta}, q e^{-u-\eta} ; q\right)_{\infty}\right|^{2}} \frac{d u}{\sinh \xi-\sinh u} \\
& =\frac{2 \pi e^{-2 \eta_{1}} N(\xi, \eta)}{\sin \eta_{2} \cosh \eta_{1} D(\xi, \eta)\left(q,-q e^{2 \eta_{1}},-q e^{-2 \eta_{1}} ; q\right)_{\infty}\left|\left(q e^{2 i \eta_{2}} ; q\right)_{\infty}\right|^{2}} .
\end{aligned}
$$

In (9.15) $\eta=\eta_{1}+i \eta_{2}$. The case $\eta_{2}=\pi / 2$ is of particular interest because the integrand in (7.30) simplifies. In (7.30) put

$$
e^{\eta}=e^{\eta_{1}+\eta_{2}}=r e^{i \pi / 2}
$$

This gives

$$
\begin{gathered}
\int_{-\infty}^{\infty} \frac{\prod_{j=1}^{4}\left(-t_{j} e^{\xi}, t_{j} e^{-\xi} ; q\right)_{\infty} \quad \cosh \xi d \xi}{\left(-r^{2} e^{2 \xi},-r^{2} e^{-2 \xi},-q^{2} r^{-2} e^{2 \xi},-q^{2} r^{-2} e^{-2 \xi} ; q^{2}\right)_{\infty}} \\
\quad=\frac{2 \pi r^{-1} \prod_{1 \leq j<k \leq 4}\left(-t_{j} t_{k} / q ; q\right)_{\infty}}{\left(-q, t_{1} t_{2} t_{3} t_{4} q^{-3},-r^{2},-q r^{-2} ; q\right)_{\infty}\left(q^{2} ; q^{2}\right)_{\infty}} .
\end{gathered}
$$

The special case $r=q^{1 / 4}$ of (9.17) is particularly interesting. It is

$$
\begin{aligned}
\int_{-\infty}^{\infty} & \frac{\prod_{j=1}^{4}\left(-t_{j} e^{\xi}, t_{j} e^{-\xi} ; q\right)_{\infty} \quad \cosh \xi d \xi}{\left(-q^{1 / 2} e^{2 \xi},-q^{1 / 2} e^{-2 \xi} ; q\right)_{\infty}} \\
\quad= & \frac{2 \pi q^{-1 / 4} \prod_{1 \leq j<k \leq 4}\left(-t_{j} t_{k} / q ; q\right)_{\infty}}{\left(-q, t_{1} t_{2} t_{3} t_{4} q^{-3},-q^{-1 / 2},-q^{-1 / 2} ; q\right)_{\infty}\left(q^{2} ; q^{2}\right)_{\infty}} .
\end{aligned}
$$

The special case $r=q^{3 / 4}$ turns out to be equivalent to the case $r=q^{1 / 4}$.

One can yet find another $q$-beta integral by choosing $g$ in (7.7) as

$$
g(z):= \begin{cases}b z+c, & \Im z>0 \\ b z+\bar{c}, & \Im z<0\end{cases}
$$


where $b \geq 0$ and $\Im c>0$. Set

$$
\sinh \eta=g(z), \quad z=\sinh \xi .
$$

Now (7.7), (9.20), and (6.25) imply

$$
\begin{aligned}
D(\sinh \eta) & {[B(z)-\sigma(z) D(z)] } \\
= & -\frac{e^{\eta}-e^{-\eta}+2 \sinh \xi}{2(q ; q)_{\infty}}\left(q e^{\xi+\eta},-q e^{\eta-\xi}, q e^{-\xi-\eta},-q e^{\xi-\eta} ; q\right)_{\infty} .
\end{aligned}
$$

An alternate representation of the right-hand side of $(9.21)$ is

$$
\begin{aligned}
& D(\sinh \eta)[B(z)-\sigma(z) D(z)] \\
& \quad=-\frac{g(z)+\sinh \xi}{(q ; q)_{\infty}} \prod_{n=1}^{\infty}\left(1-2 q^{n} g(z) e^{\xi}-q^{2 n} e^{2 \xi}\right)\left(1+2 q^{n} g(z) e^{-\xi}-q^{2 n} e^{-2 \xi}\right) .
\end{aligned}
$$

We denote by $\eta^{ \pm}$the limiting values of $\eta(z)$ as $\Im z \rightarrow 0^{ \pm}$. Clearly when $x$ is real then

$$
\begin{aligned}
& {\left[\sigma\left(x-i 0^{+}\right)-\sigma\left(x+i 0^{+}\right)\right]\left|D\left(g\left(x+i 0^{+}\right)\right)\right|^{2}} \\
& \quad=D\left(g\left(x-i 0^{+}\right)\right) B\left(g\left(x+i 0^{+}\right)\right)-B\left(g\left(x-i 0^{+}\right)\right) D\left(g\left(x+i 0^{+}\right)\right) .
\end{aligned}
$$

Apply (6.25) with $\eta$ replaced by $\eta^{-}$and $\xi$ replaced by $\eta^{+}$to arrive at

$$
\begin{aligned}
\sigma\left(x-i 0^{+}\right)-\sigma\left(x+i 0^{+}\right)= & \left(-q e^{\eta^{+}+\eta^{-}},-q e^{-\eta^{+}-\eta^{-}}, q e^{\eta^{-}-\eta^{+}}, q e^{\eta^{+}-\eta^{-}} ; q\right)_{\infty} \\
& \times \frac{\sinh \eta^{+}-\sinh \eta^{-}}{(q ; q)_{\infty}\left|D\left(x+i 0^{+}\right)\right|^{2}} .
\end{aligned}
$$

Therefore (7.8) gives

$\frac{d \psi}{d x}=\frac{\Im c}{\pi|\sinh \xi+\sinh \eta|^{2}} \frac{\left(q,-q e^{\eta^{+}+\eta^{-}},-q e^{-\eta^{+}-\eta^{-}}, q e^{\eta^{+}-\eta^{-}}, q e^{\eta^{-}+\eta^{+}} ; q\right)_{\infty}}{\left|\left(q e^{\eta^{+}+\xi},-q e^{\eta^{+}-\xi}, q e^{-\eta^{+}-\xi},-q e^{\xi-\eta^{+}} ; q\right)_{\infty}\right|^{2}}$.

It is clear from (9.24) that $d \psi / d x \geq 0$ for all real $x$ since

$$
\begin{gathered}
\left(-q e^{\eta^{+}+\eta^{-}},-q e^{-\eta^{+}-\eta^{-}}, q e^{\eta^{+}-\eta^{-}}, q e^{\eta^{-}+\eta^{+}} ; q\right)_{\infty} \\
=\left|\left(-q e^{\eta^{+}+\eta^{-}}, q e^{\eta^{-}+\eta^{+}} ; q\right)_{\infty}\right|^{2} .
\end{gathered}
$$

The expression (9.24) defines $d \psi / d x$ implicitly through $\eta^{+}$and $\eta^{-}$, so we now provide an alternate expression which exhibits the dependence on $x$ ex- 
plicitly. The sought expression is

$$
\begin{aligned}
\frac{d \psi}{d x} & =\frac{\Im c(q ; q)_{\infty}}{\pi \mid\left(x+b x+\left.c\right|^{2}\right.} \\
& \times \prod_{n=1}^{\infty} \frac{\left[1+4 q^{n}\left(1+q^{2 n}\right)|b x+c|^{2}+2 q^{2 n}\left(1-4 \Re(b x+c)^{2}\right)\right]}{\left|\left[1-4 q^{n}\left(1+q^{2 n}\right) x(b x+c)+2 q^{2 n}\left(1-2(b x+c)^{2}-2 x^{2}\right)\right]\right|^{2}} .
\end{aligned}
$$

Formula (9.26) follows from (9.24) by regrouping the terms in the infinite products.

It is important to observe that formulas (9.24) and (9.26) hold true for general functions $g(z)$ which are discontinuous almost everywhere across the real $x$ axis. The functions $\eta^{ \pm}$are such that $g\left(x \pm i 0^{+}\right)=\sinh \left(\eta^{ \pm}\right)$. Thus any function $g(z)$ which is analytic in the open upper half plane and maps it into itself and $g(\bar{z})=\overline{g(z)}$, and whose boundary values $g\left(x \pm i 0^{+}\right)$are different for almost all real $x$ gives rise to a spectral measure for the $q^{-1}$-Hermite polynomials whose absolutely continuous component is

$$
\begin{aligned}
\frac{d \psi}{d x} & =\frac{\Im g\left(x+i 0^{+}\right)(q ; q)_{\infty}}{\pi|x+g(x)|^{2}} \\
& \times \prod_{n=1}^{\infty} \frac{\left[1+4 q^{n}\left(1+q^{2 n}\right)|g(x)|^{2}+2 q^{2 n}\left(1-4 \Re g^{2}(x)\right)\right]}{\left|\left[1-4 q^{n}\left(1+q^{2 n}\right) x g(x)+2 q^{2 n}\left(1-2(g(x))^{2}-2 x^{2}\right)\right]\right|^{2}} .
\end{aligned}
$$

We now reconsider (2.10) and (2.11) in view of (5.31) and (5.35). Comparing (2.10) with (5.31) and (2.11) with (5.35) we establish the quadratic transformations

$$
\begin{aligned}
\left(-q^{1 / 2} e^{2 \xi},-q^{1 / 2} e^{-2 \xi}, q^{1 / 2}, q^{1 / 2} ; q\right)_{\infty} \\
\quad+\left(q^{1 / 2} e^{2 \xi}, q^{1 / 2} e^{-2 \xi},-q^{1 / 2},-q^{1 / 2} ; q\right)_{\infty} \\
\quad=\frac{2}{\left(q, q ; q^{2}\right)_{\infty}}\left(q e^{2 \xi}, q e^{-2 \xi} ; q^{2}\right)_{\infty}^{2}
\end{aligned}
$$

and

$$
\begin{aligned}
\left(-q^{1 / 2} e^{2 \xi},-q^{1 / 2}, e^{-2 \xi}, q^{1 / 2}, q^{1 / 2} ; q\right)_{\infty} \\
\quad-\left(q^{1 / 2} e^{2 \xi}, q^{1 / 2}, e^{-2 \xi},-q^{1 / 2},-q^{1 / 2} ; q\right)_{\infty} \\
=\frac{q^{1 / 2} \sinh ^{2} \xi}{\left(q, q ; q^{2}\right)_{\infty}}\left(q^{2} e^{2 \xi}, q^{2} e^{-2 \xi} ; q^{2}\right)_{\infty}^{2} .
\end{aligned}
$$

It is worth recording the analog of the Askey-Wilson operator [17] for the $q^{-1}$-Hermite polynomials. Define

$$
\mathscr{D}_{q} f(x):=\frac{g\left(q^{1 / 2} e^{\xi}\right)-g\left(q^{-1 / 2} e^{\xi}\right)}{\left(q^{1 / 2}-q^{-1 / 2}\right) \cosh \xi}, \quad x=\sinh \xi, g\left(e^{\xi}\right)=f(x) .
$$


We next derive an analog of the differential recurrence relation $H_{n}^{\prime}(x)=$ $2 n H_{n-1}(x)$. To do so apply $\mathscr{D}_{q}$ to the generating function (2.4) and obtain

$$
\sum_{n=0}^{\infty} \frac{q^{n(n-1) / 2} t^{n}}{(q)_{n}} \mathscr{D}_{q} h_{n}(x \mid q)=\mathscr{D}_{q}\left(-t e^{\xi}, t e^{-\xi}\right)_{\infty} .
$$

An easy calculation shows that the right-hand side of the above equality is

$$
2 t\left(-t q^{1 / 2} e^{\xi}, t q^{1 / 2} e^{-\xi}\right)_{\infty} /(1-q) \text {. }
$$

The above expression can now be expanded in a series of $q^{-1}$-Hermite polynomials using (2.4). Upon equating coefficients of like powers of $t$ we establish the relationship

$$
\mathscr{D}_{q} h_{n}(x \mid q)=2 \frac{\left(q^{-n / 2}-q^{n / 2}\right)}{\left(q^{-1 / 2}-q^{1 / 2}\right)} h_{n-1}(x \mid q) .
$$

It is well known that one cannot formally differentiate asymptotic expansions. One can, however, apply $\mathscr{D}_{q}$ to an asymptotic expansion since $\mathscr{D}_{q}$ does not involve a limiting process. For example one can obtain (5.31) from (5.35) and vice versa by applying $\mathscr{D}_{q}$ to either one and obtain the other.

\section{ACKNOWLEDGMENTS}

We thank Richard Askey for his helpful suggestions, encouragement and interest in this work. We also thank Waleed Al-Salam, Christian Berg, Mizan Rahman, and Ruiming Zhang for their comments and for providing us with relevant and useful biographical information. Last but not least we thank an anonymous referee for comments and suggestions.

\section{REFERENCES}

1. N. I. Akhiezer, The classical moment problem and some related questions in analysis, English transl., Oliver and Boyd, Edinburgh, 1965.

2. W. A. Al-Salam and L. Carlitz, Some orthogonal q-polynomials, Math. Nachr. 30 (1965), 47-61.

3. W. A. Al-Salam and T. S. Chihara, Convolutions of orthogonal polynomials, SIAM J. Math. Anal. 7 (1976), 16-28.

4. W. A. Al-Salam and M. E. H. Ismail, q-beta integrals and the q-Hermite polynomials, Pacific J. Math. 135 (1988), 209-221.

5. — A q-beta integral on the unit circle and some biorthogonal rational functions, Proc. Amer. Math. Soc. 121 (1994), 553-561.

6. W. A. Al-Salam and A. Verma, q-analogs of some biorthogonal functions, Canad. Math. Bull. 26 (1983), 225-227.

7. W. Allaway, Some properties of the q-Hermite polynomials, Canad. J. Math. 32 (1980), 686-694.

8. G. E. Andrews, q-series: their development and application in analysis, number theory, combinatorics, physics, and computer algebra, CBMS Regional Conf. Ser. in Math., vol. 66, Amer. Math. Soc., Providence, RI, 1986.

9. G. E. Andrews and R. A. Askey, Classical orthogonal polynomials, Polynomes Orthogonaux et Application (C. Breziniski et al., eds.), Lecture Notes in Math., vol. 1171, Springer-Verlag, Berlin, 1984, pp. 36-63. 
10. R. A. Askey, Beta integrals and q-extensions, Proc. Ramanujan Centenial Internat. Conf., Annamalainagar (R. Balakrishnan et al., eds.), Ramanujan Math. Soc., Annamalai University, 1988, pp. 85-102.

11. _ Beta integrals and associated orthogonal polynomials, Number Theory, Madras 1987 (K. Alladi, ed.), Lecture Notes in Math., vol. 1395, Springer-Verlag, New York, 1989, pp. 84-121.

12. - Continuous $q$-Hermite polynomials when $q>1, q$-Series and Partitions, (D. Stanton, ed)., IMA Vol. Math. Appl., Springer-Verlag, New York, 1989, 151-158.

13. R. A. Askey and M. E. H. Ismail, The very well poised ${ }_{6} \psi_{6}$, Proc. Amer. Math. Soc. 77 (1979), 218-222.

14. - The Rogers q-ultraspherical polynomials, Approximation Theory III (W. Cheny, ed.), Academic Press, New York, 1980, pp. 175-182.

15. (P. Erdös, ed.), Birkhäuser, Basel, 1983, pp. 55-78.

16. — Recurrence relations, continued fractions and orthogonal polynomials, Mem. Amer. Math. Soc. 300 (1984).

17. R. A. Askey and J. A. Wilson, Some basic hypergeometric orthogonal polynomials that generalize Jacobi polynomials, Mem. Amer. Math. Soc. 319 (1985).

18. C. Berg and G. Valent, The Nevanlinna parameterization for some indeterminate Stieltjes moment problems associated with birth and death processes, Methods and Applications of Analysis (to appear).

19. C. Brezinski, Biorthogonality and its applications to numerical analysis, Marcel Dekker, New York, 1992.

20. L. Carlitz, Generating functions for certain q-orthogonal polynomials, Collectanea Math. 23 (1972), 91-104.

21. T. S. Chihara, An introduction to orthogonal polynomials, Gordon and Breach, New York, 1978.

22. T. S. Chihara and M. E. H. Ismail, Extremal measures for a system of orthogonal polynomials, Constr. Approx. 9 (1993), 111-119.

23. D. Foata, Some Hermite polynomial identities and their combinatorics, Adv. Appl. Math. 2 (1981), 250-259.

24. G. Freud, Orthogonal polynomials, English transl., Pergamon Press, New York, 1971.

25. G. Gasper and M. Rahman, Basic hypergeometric series, Cambridge Univ. Press, Cambridge, 1990.

26. U. Grenander and G. Szegö, Toeplitz forms and their applications, Univ. of California Press, Berkely, 1958; reprinted by Chelsea, New York, 1984.

27. R. A. Gustafson, A generalization of Selberg's beta integral, Bull. Amer. Math. Soc. (N.S.) 22 (1990), 97-108.

28. M. E. H. Ismail, A queueing model and a set of orthogonal polynomials J. Math. Anal. Appl. 108 (1985), 575-594.

29. M. E. H. Ismail and D. R. Masson, Extremal measures for q-Hermite polynomials when $q>1$, C. R. Math. Rep. Acad. Sci. Canada 15 (1993), 7-12.

30. M. E. H. Ismail and D. Stanton, On the Askey-Wilson and Rogers polynomials, Canad. J. Math. 40 (1988), 1025-1045.

31. M. E. H. Ismail, D. Stanton, and G. Viennot, The combinatorics of the q-Hermite polynomials and the Askey-Wilson integral, European J. Combin. 8 (1987), 379-392.

32. M. E. H. Ismail and J. Wilson, Asymptotic and generating relations for the q-Jacobi and the ${ }_{4} \phi_{3}$ polynomials, J. Approx. Theory 36 (1982), 43-54.

33. W. F. Kibble, An extension of theorem of Mehler on Hermite polynomials, Proc. Cambridge, Philos. Soc. 41 (1945), 12-15.

34. G. Labelle, Tableau d'Askey, Polynomes Orthogonaux et Applications, (C. Breziniski et al., eds.), Lecture Notes in Math., vol. 1171, Springer-Verlag, Berlin, 1984, pp. 36-37. 
35. J. E. Littlewood, On the asymptotic approximation to integral functions of zero order, Proc. London Math. Soc. (2) 5 (1907), 361-410; reprinted in Collected Papers of J. E. Littlewood, vol. 2, Oxford Univ. Press, Oxford, 1986, pp. 1059-1109.

36. J. D. Louck, Extension of the Kibble-Slepian formula for Hermite polynomials using Boson operator methods, Adv. in Appl. Math. 2 (1981), 239-249.

37. D. Moak, The q-analogue of the Laguerre polynomials, J. Math. Anal. Appl. 81 (1981), 20-47.

38. P. Nevai, Orthogonal polynomials, Mem. Amer. Math. Soc. 13 (1979).

39. F. W. J. Olver, Asymptotics and special functions, Academic Press, New York, 1974.

40. H. Rademacher, Topics in analytic number theory, Springer-Verlag, New York, 1973.

41. M. Rahman, An integral representation of a ${ }_{10} \phi_{9}$ and continuous biorthogonal ${ }_{10} \phi_{9}$ rational functions, Canad. J. Math. 38 (1986), 605-618.

42. B Biorthogonality of a system of rational functions with respect to a positive measure on $[-1,1]$, SIAM J. Math. Anal. 22 (1991), 1421-1431.

43.

44. L. J. Rogers, On the expansion of some infinite products, Proc. London Math. Soc. 24 (1893), 337-352.

45. Math. Soc. 25 (1894), 318-343.

46. Third memoir on the expansion of certain infinite products, Proc. London Math. Soc. 26 (1895), 15-32.

47. J. Shohat and J. D. Tamarkin, The problem of moments, rev. ed., Amer. Math. Soc., Providence, RI, 1950.

48. D. Slepian, On the symmetrized Kronecker power of a matrix and extensions of Mehler's formula for Hermite polynomials, SIAM J. Math. Anal. 3 (1972), 606-616.

49. M. H. Stone, Linear transformations in Hilbert space and their application to analysis, Amer. Math. Soc., Providence, RI, 1932.

50. G. Szegö, Orthogonal polynomials, 4th ed., Amer. Math. Soc., Providence, RI, 1975.

51. M. Toda, Theory of nonlinear lattices, 2nd enlarged ed., Springer-Verlag, Berlin, 1988.

52. E. T. Whittaker and G. N. Watson, A course of modern analysis, 2nd ed., Cambridge Univ. Press, Cambridge, 1927.

Department of Mathematics, University of South Florida, TAMPa, Florida 33620-5700

E-mail address: ismail@math.usf.edu

Department of Mathematics, University of Toronto, Toronto, Ontario, Canada M5S $1 \mathrm{~A} 1$

E-mail address: masson@math.toronto.edu 